\title{
ASYMPTOTIC EXPANSION FOR LAYER SOLUTIONS OF A SINGULARLY PERTURBED REACTION-DIFFUSION SYSTEM
}

\author{
XIAO-BIAO LIN
}

\begin{abstract}
For a singularly perturbed $n$-dimensional system of reactiondiffusion equations, assuming that the 0th order solutions possess boundary and internal layers and are stable in each regular and singular region, we construct matched asymptotic expansions for formal solutions in all the regular, boundary, internal and initial layers to any desired order in $\epsilon$. The formal solution shows that there is an invariant manifold of wave-front-like solutions that attracts other nearby solutions. We also give conditions for the wave-front-like solutions to converge slowly to stationary solutions on that manifold.
\end{abstract}

\section{INTRODUCTION}

This is the first of a series of papers devoted to studying internal, boundary and initial layers for singularly perturbed $n$-dimensional systems of reaction-diffusion equations. By a formal asymptotic method, we derive matched expansions of layer solutions to any desired order in $\epsilon$. We give general conditions for existence and stability of the formal solutions. The formal expansion shows how the initial profile quickly converges to a manifold of slow moving wave-front-like solutions. We also give an analytic condition (see Hypothesis H6) for the formal solution to converge slowly towards a stable stationary solution. In the next paper we will show that under the same set of conditions there is a unique genuine solution that is near the formal series solution. These results have been obtained by other authors for $n=1$, or $n=2$ with small diffusion on only one variable. Our goal is to generalize their results to any finite $n$.

Obtaining matched asymptotic expansion has always been an indispensable part of the complete treatment of singular perturbation problems, for it often provides easily computable and highly accurate approximations to the exact solutions. However, a rigorous treatment of the asymptotic expansion to the layer solutions of the general systems has remained incomplete for almost twenty years since first raised by Fife $[12,13]$. We have also noted that new tools need to be developed when moving from scalar equations to systems. Recent advances using the Melnikov integrals in the functional analytic method of homoclinic bifurcations are crucial to this paper, see $[4,26,22,23]$.

Received by the editors July 5, 1994 and, in revised form, January 13, 1995.

1991 Mathematics Subject Classification. Primary 35K57, 35B25; Secondary 34E10, 34E15.

Key words and phrases. Asymptotic expansions, singular perturbations, reaction-diffusion systems, boundary-internal and initial layers, stability.

Research partially supported by NSFgrant DMS9002803 and DMS9205535. 
Consider the following reaction-diffusion equation

$$
\epsilon u_{t}=\epsilon^{2} u_{x x}+f(u, x, \epsilon), \quad u \in \mathbb{R}^{n}, a<x<b,
$$

with Neumann boundary conditions at $x=a, b$

$$
u_{x}(a, t)=u_{x}(b, t)=0,
$$

and initial condition

$$
u(x, 0, \epsilon)=\bar{u}(x, \epsilon)
$$

at $t=0$. Here $f: \mathbb{R}^{n} \times[a, b] \times \mathbb{R} \rightarrow \mathbb{R}^{n}$ is $C^{\infty}$ with the following expansion:

$$
f(u, x, \epsilon)=\sum_{j=0}^{\infty} \epsilon^{j} f_{j}(u, x) .
$$

Due to the presence of the small parameter $\epsilon>0$, solutions of (1.1) may have internal, boundary and initial layers. Those are the regions of $x$ - $t$ space where $u_{x x}$ and/or $u_{t}$ are large so that the solutions do not converge uniformly as $\epsilon \rightarrow 0$. For the moment we ignore boundary layers, and give a short introduction to spatially regular and internal layers. The following is motivated by a discussion in $[12,13]$. of

In the regular layers, a stationary solution $u(x, \epsilon)$ of (1.1) approaches solutions

$$
f_{0}(u, x)=0
$$

as $\epsilon \rightarrow 0$. Assume that $u=p^{i}(x), i=1,2, a \leq x \leq b$, are two solutions of (1.4), and as $\epsilon \rightarrow 0$,

$$
u(x, \epsilon) \rightarrow \begin{cases}p^{1}(x), & a<x<\eta, \\ p^{2}(x), & \eta<x<b .\end{cases}
$$

There is an internal layer at $x=\eta$. Using stretched variables $\xi=\frac{x-\eta}{\epsilon}, \tau=\frac{t}{\epsilon}$, we write (1.1) as (when $\epsilon=0$ )

$$
u_{\tau}=u_{\xi \xi}+f_{0}(u, \eta)
$$

Suppose that $\eta=\eta^{0}$ can be chosen such that (1.6) possesses a stationary solution $q(\xi)$ that satisfies

$$
0=u_{\xi \xi}+f_{0}(u, \eta)
$$

and approaches $p^{1}\left(\eta^{0}\right)$ as $\xi \rightarrow-\infty\left(p^{2}\left(\eta^{0}\right)\right.$ as $\left.\xi \rightarrow \infty\right), q^{\prime}(\xi) \rightarrow 0$ as $\xi \rightarrow \pm \infty$. The functions $\left\{p^{1}(x), q(\xi), p^{2}(x)\right\}$ are the 0th order expansion of a formal solution in regular and internal layers. The position of the stationary internal layer $\eta=\eta_{0}$ is determined by the existence of a heteroclinic solution to (1.7). The condition $q(\xi) \rightarrow p^{i}\left(\eta^{0}\right)$ as $\xi \rightarrow \pm \infty$ is the 0 th matching condition between regular and singular layers. Higher order matching conditions will be specified later in this paper when higher order formal expansions are computed.

Under some general conditions, which will be stated in $\S 3$, it was proved $[22,23]$ that there is an exact stationary solution $u$ to (1.1) near the 0th order expansions. Similar results were obtained in [21].

When $\eta$ changes, generically the heteroclinic solution of (1.7) breaks. However the time dependent equation (1.6) may have a traveling wave solution $u(\xi, \tau)=$ $q(\xi-V \tau, \eta)$ where $q(\xi, \eta)$ satisfies

$$
u_{\xi \xi}+V u_{\xi}+f_{0}(u, \eta)=0
$$


Here $\eta$ serves as a parameter, and the wave speed $V=V(\eta)$ depends on $\eta$. The function $q(\xi, \eta)$ approaches one of the $p^{i}(\eta)$, and $\frac{\partial}{\partial \xi} q$ approaches zero, as $\xi \rightarrow \pm \infty$ due to the matching of the internal and regular layers. The wave speed $V$ and the wave front position $\eta$ do not depend on the stretched time $\tau$, but they depend on the slow time $t$. To see this, let $\eta=\eta(t), \xi=\frac{x-\eta(t)}{\epsilon}$, and $u\left(\frac{x-\eta(t)}{\epsilon}, t\right)=u(\xi, t)$ be a solution to (1.1). Then (when $\epsilon=0$ )

$$
0=u_{\xi \xi}+\eta^{\prime}(t) u_{\xi}+f_{0}(u, \eta(t))
$$

At each $t \geq 0$, we look for a heteroclinic solution of the above connecting $p^{1}(\eta(t))$ to $p^{2}(\eta(t))$. Comparing this with (1.8), we have

$$
\frac{d \eta(t)}{d t}=V(\eta(t))
$$

We can see that (1.10) determines $\eta(t)$. One should not be surprised to see that the wave speed $V$ is the same in both $x$ - $t$ and $\xi-\tau$ coordinates, since the scaling by $\epsilon$ cancels.

We have just described the wave-front-like solutions to the 0th order. Recursive formulas for computing higher order expansions of $u$ and $\eta$ are also presented in this paper. In particular, we have found that the higher order expansions in the internal layers are uniquely determined by growth conditions of the solutions while the matching of such solutions with those in adjacent regular layers can be proved as a consequence of that. See [22] for a similar case.

Let $0<\beta<1$ be a constant. Let the width of the internal layer be $O\left(\epsilon^{\beta}\right)$. The variable $x=\epsilon^{\beta}$ is $o(1)$ as $\epsilon \rightarrow 0$. But in the stretched variable, $\xi=x / \epsilon=\epsilon^{\beta-1} \rightarrow \infty$ as $\epsilon \rightarrow 0$. See $[6,7]$ for discussion of such intermediate variables. Define a piecewise smooth function $W(x, t, \epsilon)$ by

$$
W(x, t, \epsilon)= \begin{cases}p^{1}(x), & a<x<\eta(t)-\epsilon^{\beta}, \\ p^{2}(x), & \eta(t)+\epsilon^{\beta}<x<b, \\ q((x-\eta(t)) / \epsilon), & \eta(t)-\epsilon^{\beta}<x<\eta(t)+\epsilon^{\beta} .\end{cases}
$$

At the interior of each subinterval, $W$ satisfies (1.1) with an error $O\left(\epsilon^{\beta}\right)$. At the points $\eta(t) \pm \epsilon^{\beta}, W$ has a jump discontinuity of size $O\left(\epsilon^{\beta}\right)$. Such a function is called a pseudo-solution to (1.1). In our next paper we will show that there is an exact solution to the original equation (1.1) that is near $W(x, t, \epsilon)$. A function is said to have a wave-front-like profile or to be a wave-front-like function if it approaches the solutions of (1.4) at regular layers, but approaches heteroclinic solutions in stretched variable at internal layers. The function $W$ obviously has a wave-frontlike profile. It follows that the exact solution of (1.1) near $W$ has a wave-front-like profile.

Recall that $V\left(\eta_{0}\right)=0$. The wave speed $V(\eta)$ generally changes sign when passing $\eta=\eta^{0}$. The case $V<0$ if $\eta>\eta^{0}$ is especially interesting. It shows that the wavefront-like solution approaches a stationary wave-front-like solution as time evolves. In the other case $V>0$ when $\eta>\eta^{0}$, the stationary wave-front-like solution is not stable among the wave-front-like solutions.

Suppose now the initial condition $\bar{u}(x, \epsilon)$ also has a wave-front-like profile. That is, $\bar{u}(x, 0)$ has a jump at $x=\eta$ and is continuous on $[a, \eta)$ and $(\eta, b]$, and using the stretched variable $\xi=\frac{x-\eta}{\epsilon}$, the limit $\lim _{\epsilon \rightarrow 0^{+}} \bar{u}(\epsilon \xi+\eta, \epsilon)=\overline{\bar{u}}(\xi, 0)$ exists. We assume that as initial data for the ODE $u_{\tau}=f_{0}(u, x)$, where $x$ serves as a parameter, $\bar{u}(x, 0)$ is attracted by $p^{i}(x), i=1$ for $x \in[a, \eta), i=2$ for $x \in(\eta, b]$, and 
as initial data for (1.6), $\overline{\bar{u}}(\xi, 0)$ is attracted by $q(\xi, \eta)$ modulo a spatial shift. $q(\xi, \eta)$ is stable in the sense of Evans, $[8,9,10,11]$. The problem of determining when $q(\xi, \eta)$ is stable is very important, but is not the concern of this paper, $[20,2,25,19]$.

Under the above assumptions, using stretched time $\tau=t / \epsilon$, we also derive formal series solutions in the initial layers. Special care has to be exercised to ensure that these solutions match with wave-front-like slow solutions, since the linearized equation has a zero eigenvalue.

In this paper, the intermediate spaces $D_{A}(\theta)$ are used to study parabolic equations. These spaces are powerful tools to treat fully nonlinear equations. Since our system is semi-linear, we only use some weaker results. All the results in this paper are valid with essentially the same proof if $D_{A}(\theta)$ is replaced by $D\left(A^{\theta}\right)$. An important feature in this paper is to use weighted norms in function spaces. Weighted function spaces have been used in $[28,29]$ to study the stability of travelling waves.

The outline of this paper is as follows. We introduce notations and some basic lemmas in $\S 2$. In $\S 3$ we state a result from [22] that concerns the stationary wavefront-like formal series solution (Theorem 3.1). The result in $\S 3$ is a special case of the result in $\S 4$. Having a separate section helps to show what new hypotheses are needed to study the slowly moving wave-front-like solutions. In $\S 4$, we study wavefront-like formal series solution (Theorems 4.3, 4.4, and Corollary 4.5). We show that these solutions have a slowly moving front and form a manifold that depends on parameters $\left\{\bar{\eta}_{j}^{i}\right\}_{j=0}^{\infty}, 1 \leq i \leq r-1$. Here $\sum_{0}^{\infty} \epsilon^{j} \bar{\eta}_{j}^{i}$, is the formal series expansion of the initial wave front, $r-1$ is the number of internal layers. The result agrees with other publications where the slow manifold is also parameterized by layer positions, $[3,17,19,1]$. In $\S 5$ we study the formal series solutions in the initial layer (Theorems 5.3, 5.5). We show that $\bar{\eta}_{0}^{i}$ is determined by the initial condition of (1.1), and $\bar{\eta}_{j}^{i}, j \geq 1$, are determined by the matching of initial and regular (in time) expansions. In $\S 6$, we prove that the formal series solutions in adjacent layers, obtained in $\S 4$ and $\S 5$, match with each other (Theorems 6.1-6.4). We also construct a pseudo-solution of any prescribed accuracy based on the matched series solutions (Theorem 6.5). Most of the technical lemmas are proved in $\S 7$.

Internal and boundary layers in singular perturbation problems have been an active area of current research. Various powerful methods have been developed to treat the layer solutions $[14,16,15,25]$. Our approach is different from the others. We follow the pattern "matched formal series expansions-pseudo solutionsNewton's method" which has been used to treat singularly perturbed ODEs, see $[22,23]$. The last step uses a lemma similar to the shadowing lemma in dynamical system theory. In our next paper we will introduce such a shadowing lemma for parabolic systems where the pseudo-solutions have jumps in both $x$ and $t$ directions. A brief look at such a result reveals that the inverse operator of the parabolic system has a norm of $O\left(\epsilon^{-k}\right), k>1$. A good initial approximation with residual smaller than $O\left(\epsilon^{k}\right)$ is needed which is naturally supplied by the matched asymptotic expansions.

1.1. Acknowledgment. It is a pleasure to thank the referee for his valuable suggestions which resulted in an improvement of the manuscript.

\section{NotATIONS AND BASIC LEMMAS}

2.1. Notations. As $\epsilon \rightarrow 0$, the solution $u(x, t, \epsilon)$ of (1.1) may not converge uniformly at regions where $u_{x x}$ and $u_{t}$ are large. These regions are called singular layers 


\begin{tabular}{c|c|c|c|c|c|c|c|c}
$t$ & $(S R)^{0}$ & $(R R)^{1}$ & $\ldots$ & $(R R)^{i}$ & $(S R)^{i}$ & $(R R)^{i+1}$ & $\ldots$ & $(S R)^{r}$ \\
\hline$\tau$ & $(S S)^{0}$ & $(R S)^{1}$ & $\ldots$ & $(R S)^{i}$ & $(S S)^{i}$ & $(R S)^{i+1}$ & $\ldots$ & $(S S)^{r}$ \\
\hline & $\xi$ & $x$ & $\ldots$ & $x$ & $\xi$ & $x$ & $\ldots$ & $\xi$
\end{tabular}

FIGURE 1

with respect to space or time. In particular, $u_{t}$ may be large near $t=0$. That region is also called the fast (or initial) layer where the stretched time $\tau=t / \epsilon$ is appropriate to express the solutions. $u_{x}$ and $u_{x x}$ may be large near some $x=\eta^{i}, 0 \leq i \leq r$. These regions are called boundary (internal) layers if $i=0, r$ (or $1 \leq i \leq r-1$ ), where the stretched space variable $\xi=\frac{x-\eta^{i}}{\epsilon}$ is used. Regions that are not singular with respect to space or time are called regular layers. We use $S$ or $R$ to denote singular or regular layers. The symbol related to space is put before that related to time since in the dictionary order space is before time. Thus $(S R)^{i},(R R)^{i},(S S)^{i}$ and $(R S)^{i}$ are used to denote the $i$ th spatially singular, temporally regular region, etc. Figure 1 shows relative locations of all the possible layers. Superscripts on a solution are used to show the type of layers where the solution is expressed by the appropriate variables.

$$
\begin{array}{llll}
u^{R R i}(x, t, \epsilon) & =u(x, t, \epsilon), & & \text { for }(x, t) \in(R R)^{i}, \\
u^{S R i}(\xi, t, \epsilon) & =u\left(\epsilon \xi+\eta^{i}, t, \epsilon\right), & & \text { for }(x, t) \in(S R)^{i}, \\
u^{R S i}(x, \tau, \epsilon) & =u(x, \epsilon \tau, \epsilon), & & \text { for }(x, t) \in(R S)^{i}, \\
u^{S S i}(\xi, \tau, \epsilon) & =u\left(\epsilon \xi+\eta^{i}, \epsilon \tau, \epsilon\right), & & \text { for }(x, t) \in(S S)^{i} .
\end{array}
$$

Each layers is further expanded in powers of $\epsilon, u^{R R i}(x, t, \epsilon)=\sum_{j=0}^{\infty} \epsilon^{i} u_{j}^{R R i}(x, t)$, etc.

The notation $\stackrel{*}{u}(\tau)$ is used to denote the expansion of $u(t)$ in the variable $\tau$. $\tilde{u}(\xi)$ is used to denote the expansion of $u(x)$ in the variable $\xi . \bar{u}$ is used to denote the initial condition for a solution $u$.

Let $C_{b u}\left(\mathbb{R}, \mathbb{R}^{n}\right), C_{b u}\left(\mathbb{R}^{+}, \mathbb{R}\right)$ and $C_{b u}\left(\mathbb{R}^{-}, \mathbb{R}^{n}\right)$ be the Banach spaces of uniformly continuous and bounded functions with super norms. Let $C_{b u}^{m}=\left\{u \mid u, u^{\prime}, \ldots, u^{(m)}\right.$ $\left.\in C_{b u}\right\}$ with the norm

$$
\|u\|_{C_{b u}^{m}}=\sum_{i=0}^{m}\left\|u^{(i)}\right\|_{C_{b u}} .
$$

Here $C_{b u}^{m}$ denotes $C_{b u}^{m}\left(\mathbb{R}, \mathbb{R}^{n}\right)$ or $C_{b u}^{m}\left(\mathbb{R}^{ \pm}, \mathbb{R}^{n}\right)$. We can show that $C_{b u}^{m}, m \geq 1$, is dense in $C_{b u}^{m-1}$.

For a continuous function $w(\xi)>0$, let $E_{\mathbb{R}}(w)$ be the Banach space of functions with the weight $w(\xi)$.

$$
\begin{gathered}
E_{\mathbb{R}}(w)=\left\{u: \mathbb{R} \rightarrow \mathbb{R}^{n} \mid u(\cdot) / w(\cdot) \in C_{b u}\left(\mathbb{R}, \mathbb{R}^{n}\right)\right\} . \\
\|u\|_{E(w)}=\sup \{|u(\xi) / w(\xi)|, \xi \in \mathbb{R}\} \\
E_{\mathbb{R}}^{m}(w)=\left\{u \mid u, \ldots, u^{(m)} \in E(w)\right\} . \\
\|u\|_{E^{m}(w)}=\sum_{j=0}^{m}\left\|u^{(j)}\right\|_{E(w)} .
\end{gathered}
$$

Similarly, $E_{\mathbb{R}^{+}}^{m}(w)$ and $E_{\mathbb{R}^{-}}^{m}(w)$ are Banach spaces of weighted functions that are defined on $\mathbb{R}^{+}$and $\mathbb{R}^{-}$. We use $E^{m}(w)$ to denote $E_{\mathbb{R}}^{m}(w)$ or $E_{\mathbb{R}^{ \pm}}^{m}(w)$ if no confusion 
should arise. One of the most often used weights is

$$
w(\xi)=\left(1+|\xi|^{j}\right) e^{-\gamma \xi}, \quad \gamma \in \mathbb{R}, j \geq 0 .
$$

Let

$B_{\mathbb{R}^{+}}^{m}=\left\{u \in C_{b u}^{m}\left(\mathbb{R}^{+}, \mathbb{R}^{n}\right), \lim _{\xi \rightarrow \infty} D_{\xi}^{\alpha} u(\xi)=u^{(\alpha)}(+\infty)\right.$ exists for $\left.\alpha \leq m\right\}$,

$B_{\mathbb{R}^{-}}^{m}=\left\{u \in C_{b u}^{m}\left(\mathbb{R}^{-}, \mathbb{R}^{n}\right), \lim _{\xi \rightarrow-\infty} D_{\xi}^{\alpha} u(\xi)=u^{(\alpha)}(-\infty)\right.$ exists for $\left.\alpha \leq m\right\}$.

$B_{\mathbb{R}}^{m}=\left\{u \in C_{b u}^{m}\left(\mathbb{R}, \mathbb{R}^{n}\right), \lim _{\xi \rightarrow \pm \infty} D_{\xi}^{\alpha} u(\xi)=u^{(\alpha)}( \pm \infty)\right.$ exists for $\left.\alpha \leq m\right\}$.

One can easily verify that $u^{(\alpha)}( \pm \infty)=0$ if $\alpha \geq 1$.

Let $w(\xi)=\left(1+|\xi|^{j}\right) e^{-\gamma|\xi|}, \gamma>0$. Define,

$$
\begin{array}{ll}
B_{\mathbb{R}^{+}}^{m}(w)=\left\{u \in B_{\mathbb{R}^{+}}^{m},\right. & \left.u(\cdot)-u(+\infty) \in E_{\mathbb{R}^{+}}^{m}(w)\right\} . \\
B_{\mathbb{R}^{-}}^{m}(w)=\left\{u \in B_{\mathbb{R}^{-}}^{m},\right. & \left.u(\cdot)-u(-\infty) \in E_{\mathbb{R}^{-}}^{m}(w)\right\} . \\
B_{\mathbb{R}^{m}}^{m}(w)=\left\{u \in B_{\mathbb{R}^{m}}^{m},\right. & \left.u(\cdot)-u( \pm \infty) \in E_{\mathbb{R}^{ \pm}}^{m}(w)\right\} .
\end{array}
$$

The general notation $B^{m}(w)$ will be used if no confusion should arise. Let the norms of $B_{\mathbb{R}^{+}}^{m}, B_{\mathbb{R}^{-}}^{m}, B_{\mathbb{R}}^{m}$ be induced from $C_{b u}^{m}$ respectively. It is clear that $B_{\mathbb{R}^{+}}^{m}, B_{\mathbb{R}^{-}}^{m}$ and $B_{\mathbb{R}}^{m}$ are Banach spaces with these norms. Let

$$
\|u\|_{B_{\mathbb{R}^{m}}^{m}(w)}=\|u\|_{C_{b u}^{m}}+\|u-u(+\infty)\|_{E_{\mathbb{R}^{+}}^{m}(w)}+\|u-u(-\infty)\|_{E_{\mathbb{R}^{-}}^{m}(w)}
$$

Similarly definitions are given to $\|u\|_{B_{\mathbb{R}^{+}}^{m}}(w)$ and $\|u\|_{B_{\mathbb{R}^{-}}^{m}}(w)$. It can be verified that $B_{\mathbb{R}^{m}}^{m}(w), B_{\mathbb{R}^{-}}^{m}(w)$ and $B_{\mathbb{R}^{+}}^{m}(w)$ are all Banach spaces with the specified norms.

Let $X$ be one of the Banach spaces: $E_{\mathbb{R}^{+}}^{m}(w), E_{\mathbb{R}^{-}}^{m}(w), B_{\mathbb{R}^{+}}^{m}(w)$ or $B_{\mathbb{R}^{-}}^{m}(w)$, with $m \geq 1$. For $C^{1}$ functions defined on $\mathbb{R}^{+}$or $\mathbb{R}^{-}$, denote $B C=\left\{u \in C^{1}: u_{\xi}(0)=0\right\}$. $X \cap(B C)$ is a closed subspace of $X$, and a Banach space with the norm induced from $X$.

The following lemma is useful when working with these weighted spaces and can be verified easily.

Lemma 2.1. Let $\alpha>0,|\gamma|<\alpha$ be real constants, $j \geq 0$ be an integer. Then there exists a constant $K_{1}$ such that

$$
\begin{aligned}
& \int_{-\infty}^{\xi} e^{-\alpha(\xi-s)}\left(1+|s|^{j}\right) e^{-\gamma s} d s+\int_{\xi}^{\infty} e^{-\alpha(s-\xi)}\left(1+|s|^{j}\right) e^{-\gamma s} d s \\
& \quad \leq \frac{K_{1} e^{-\gamma \xi}}{(\alpha-|\gamma|)^{j+1}}\left(1+|\xi|^{j}\right) .
\end{aligned}
$$

2.2. Properties of elliptic equations. Assume that $f: \mathbb{R}^{n} \rightarrow \mathbb{R}^{n}$ is $C^{\infty}$, and $V$ is a real constant. Equation

$$
u_{\xi \xi}+V u_{\xi}+f(u)=0
$$

is equivalent to a system in $\mathbb{R}^{2 n}$ :

$$
\begin{aligned}
& u_{\xi}=v, \\
& v_{\xi}=-V v-f(u) .
\end{aligned}
$$

Therefore the phase space for $(2.4)$ is $\mathbb{R}^{2 n}$, comprised of points $\left(u, u_{\xi}\right)$. We say $p$ is a hyperbolic equilibrium for $(2.4)$ if $(p, 0)$ is a hyperbolic equilibrium for $(2.5)$. We say equation

$$
u_{\xi \xi}+V u_{\xi}+A(\xi) u=0
$$


has exponential dichotomy on an interval $I \subset \mathbb{R}$ if the system

$$
\begin{aligned}
& u_{\xi}=v, \\
& v_{\xi}=-V v-A(\xi) u
\end{aligned}
$$

has an exponential dichotomy on $I$. Here $A(\cdot): I \rightarrow \mathbb{R}^{n \times n}$ is a continuous matrix valued function.

We say $u(\xi)$ is a heteroclinic solution of $(2.4)$ if $\left(u(\xi), u_{\xi}(\xi)\right)$ is a heteroclinic solution for the equivalent system (2.5).

Lemma 2.2. Assume that $p \in \mathbb{R}^{n}, f: \mathbb{R}^{n} \rightarrow \mathbb{R}^{n}$ is $C^{\infty}$ and there exists $\sigma_{0}>0$

$$
f(p)=0, \quad R_{e} \sigma\{D f(p)\} \leq-\sigma_{0} .
$$

Then

$$
u_{\xi \xi}+V u_{\xi}+D f(p) u=0
$$

has an exponential dichotomy on $\mathbb{R}$ with $n$-dimensional stable and unstable spaces. Let $0<\alpha<\sqrt{V^{2}+4 \sigma_{0}}-|V|$. Then the decay rate on the stable (or unstable) subspace is bounded by $K e^{-\alpha \xi}, \xi \geq 0$ (or $K e^{\alpha \xi}, \xi \leq 0$ ) respectively.

Let $p^{i}, i=1,2$, satisfy (2.8). Let $q(\xi)$ be a solution to (2.4) and is defined on $\mathbb{R}^{-}$ with $q(\xi) \rightarrow p^{1}$ as $\xi \rightarrow-\infty$, and/or is defined on $\mathbb{R}^{+}$with $q(\xi) \rightarrow p^{2}$ as $\xi \rightarrow \infty$. Then

$$
u_{\xi \xi}+V u_{\xi}+D f(q(\xi)) u=0
$$

has exponential dichotomies on $\mathbb{R}^{-}$or $\mathbb{R}^{+}$respectively, with $\mathcal{R} P_{s}(t)$ and $\mathcal{R} P_{u}(t)$ being $n$-dimensional subspaces in $\mathbb{R}^{2 n}$. Here $P_{u}(t)+P_{s}(t)=I, t \in \mathbb{R}^{-}$or $t \in \mathbb{R}^{+}$, are the projections to the unstable and stable subspaces. Moreover, the decay rate $\alpha>0$ is the same as that of (2.9). In the case that $q$ is a heteroclinic solution connecting $p^{1}$ and $p^{2}, \mathcal{R} P_{u}\left(0^{-}\right) \cap \mathcal{R} P_{s}\left(0^{+}\right)$is at least one dimensional, containing $\left(q_{\xi}(0), q_{\xi \xi}(0)\right)$.

Lemma 2.3. (i) Let $p \in \mathbb{R}^{n}$ satisfy (2.8), $\alpha$ be the constant as in Lemma 2.2. Let $w(\xi)$ be the weight function in (2.2) where $|\gamma|<\alpha, X=E_{\mathbb{R}}^{m}(w)$ and $g \in X$. Then there exists a unique solution $u \in E_{\mathbb{R}}^{m+2}(w)$ to the equation

$$
u_{\xi \xi}+V u_{\xi}+D f(p) u=g .
$$

Moreover,

$$
\|u\|_{E^{m+2}(w)} \leq C\|g\|_{E^{m}(w)} .
$$

(ii) Let $X$ be $E_{\mathbb{R}^{+}}^{m}(w)$ or $E_{\mathbb{R}^{-}}^{m}(w)$, and $g \in X$. Assume that $p^{i}, i=1,2$, and $q(\xi)$ are as in Lemma 2.2. Assume that $u_{\xi}(0) \neq 0$ for all nontrivial bounded solutions $u$ to the equation $u_{\xi \xi}+V u_{\xi}+D f(q) u=0$. Then there exists a unique solution $u \in E_{\mathbb{R}^{+}}^{m+2}(w)$ or $E_{\mathbb{R}^{-}}^{m+2}(w)$ to the boundary value problem

$$
\begin{aligned}
& u_{\xi \xi}+V u_{\xi}+D f(q) u=g, \\
& u_{\xi}(0)=\phi,
\end{aligned}
$$

Moreover,

$$
\|u\|_{E^{m+2}(w)} \leq C\left(\|g\|_{E^{m}(w)}+\|\phi\|_{\mathbb{R}^{n}}\right) .
$$


Let $p^{i} \in \mathbb{R}^{n}, i=1,2$, satisfy (2.8). Let $q(\xi)$ be a heteroclinic solution to (2.4) connecting $p^{1}$ to $p^{2}$. Let $X=E_{\mathbb{R}}^{m}(w)$ where $w(\xi)$ is as in Lemma 2.3. Define $L_{q}: X \rightarrow X$ with $D\left(L_{q}\right)=E_{\mathbb{R}}^{m+2}(w)$ by

$$
L_{q} u=u_{\xi \xi}+V u_{\xi}+D f(q(\xi)) u .
$$

Lemma 2.4. $L_{q}$ is a Fredholm operator with Fredholm index zero. Assume that $\operatorname{dim} \operatorname{Ker}\left(L_{q}\right)=1$ then $\operatorname{Ker}\left(L_{q}\right)=\operatorname{span}\left\{q_{\xi}\right\}$ and Range $\left(L_{q}\right)=\{\Psi\}^{\perp}$. Here $\Psi$ is the unique nontrivial bounded solution for the adjoint equation, up to a scalar multiple,

$$
\begin{gathered}
L_{q}^{*} \Psi \stackrel{\text { def }}{=} \Psi_{\xi \xi}-V \Psi_{\xi}+D f^{\tau}(q(\xi)) \Psi=0 . \\
\{\Psi\}^{\perp}=\left\{u \in X \mid \int_{-\infty}^{\infty} \Psi^{\tau}(\xi) u(\xi) d \xi=0\right\} .
\end{gathered}
$$

2.3. Properties of Parabolic Equations. Let $A$ be a closed densely defined linear operator in a Banach space $X$. Suppose that $A$ is sectorial and generates a $C^{0}$ analytic semigroup $e^{A t}$ in $X$. For $0 \leq \theta<1$, let $D_{A}(\theta)$ be the intermediate space between $D_{A}$ and $X$.

$$
\begin{array}{ll}
D_{A}(\theta) & =\left\{x \in X \mid \lim _{t \rightarrow 0} t^{1-\theta} A e^{A t} x=0\right\}, \\
\|x\|_{\theta} & =\sup _{0<t \leq 1}\left|t^{1-\theta} A e^{A t} x\right|_{X}+|x|_{X}, \\
D_{A}(\theta+1) & =\left\{x \mid x \in D_{A}, A x \in D_{A}(\theta)\right\} .
\end{array}
$$

Let $D_{A}(1)=D_{A}$. Let $0 \leq \beta \leq \theta \leq 1$ and $0 \leq \theta-\beta<1$. Let $F: D_{A}(\theta) \rightarrow D_{A}(\beta)$ be a nonlinear, Lipschitz continuous function and $x \in D_{A}(\theta), 0 \leq \theta \leq 1$. Then there exist $t_{0}>0$ and a unique classical solution $u$ defined on $\left[0, t_{0}\right]$ such that

$$
\begin{aligned}
u_{t} & =A u+F(u), \\
u(0) & =x,
\end{aligned}
$$

where

$$
u \in C\left(\left[0, t_{0}\right]: D_{A}(\theta)\right) \cap C^{1}\left(\left(0, t_{0}\right]: X\right) \cap C\left(\left(0, t_{0}\right]: D_{A}\right) .
$$

Denote the solution by $U(t)$. Then $U:\left[0, t_{0}\right] \rightarrow D_{A}(\theta)$ is Hölder continuous with Hölder exponent $1+\beta-\theta$. Assume that $F \in C^{1}$ with $D F$ being Lipschitz continuous. Then $D F(U(\cdot)):\left[0, t_{0}\right] \rightarrow \mathcal{L}\left(D_{A}(\theta): D_{A}(\beta)\right)$ is Hölder continuous. Let $g:\left[0, t_{0}\right] \rightarrow D_{A}(\beta)$ be locally Hölder continuous, then

$$
\begin{aligned}
u_{t} & =A u+D F(U(t)) u+g(t), \\
u(0) & =u_{0},
\end{aligned}
$$

has a unique classical solution $u$ that also satisfies (2.17). Moreover there exists an evolution operator $T(t, s)$ such that

$$
u(t)=T(t, 0) u_{0}+\int_{0}^{t} T(t, s) g(s) d s .
$$

These well known facts can be found in [5], [31] and [24].

The linear equation (2.18) is said to be exponentially stable if $T(t, s)$ is defined for all $0 \leq s \leq t$ and if there exist constants $K, \alpha>0,0 \leq \theta-\beta<1$, such that

$$
\begin{aligned}
\|T(t, s)\|_{\theta} & \leq K e^{-\alpha(t-s)}, \quad t>s ; \\
\|T(t, s)\|_{\mathcal{L}\left(D_{A}(\beta): D_{A}(\theta)\right)} & \leq K\left[1+(t-s)^{\beta-\theta}\right], \quad t>s .
\end{aligned}
$$


Lemma 2.5. Assume that (2.18) is exponentially stable and $g:[0, \infty) \rightarrow D_{A}(\beta)$ is locally Hölder continuous and for some integer $k \geq 0$,

$$
\|g(t)\|_{D_{A}(\beta)} \leq C\left(1+t^{k}\right), \quad t \geq 0
$$

Then for each $u_{0} \in D_{A}(\theta)$, (2.18) has a unique solution that satisfies (2.17) for all $t_{0}>0$ and

$$
\|u(t)\|_{\theta} \leq C\left(1+t^{k}\right), \quad t \geq 0 .
$$

Let $w(\xi)$ be a weight function in $(2.2)$. Let $X=E_{\mathbb{R}}(w), E_{\mathbb{R}^{+}}(w), E_{\mathbb{R}^{-}}(w)$, $B_{\mathbb{R}}(w), B_{\mathbb{R}^{+}}(w)$ or $B_{\mathbb{R}^{-}}(w)$. Let $A: X \rightarrow X$ be defined as

$$
A u=u_{\xi \xi}
$$

with $D_{A}=E_{\mathbb{R}}^{2}(w)$ or $B_{\mathbb{R}}^{2}(w)$ if $X=E_{\mathbb{R}}(w)$ or $B_{\mathbb{R}}(w), D_{A}=E_{\mathbb{R}^{ \pm}}^{2}(w) \cap B C$ or $B_{\mathbb{R}^{ \pm}}^{2}(w) \cap B C$ if $X=E_{\mathbb{R}^{ \pm}}(w)$ or $B_{\mathbb{R}^{ \pm}}(w)$.

Lemma 2.6. $A$ is a sectorial operator in $X$ with $D_{A}$ dense in $X$.

Lemma 2.7. Let $X$ and $A: D_{A} \rightarrow X$ be as in Lemma 2.6. Then depending on the choice of $X, E_{\mathbb{R}}^{1}(w)$, or $B_{\mathbb{R}}^{1}(w)$, or $E_{\mathbb{R}^{ \pm}}^{1}(w) \cap B C$, or $B_{\mathbb{R}^{ \pm}}^{1}(w) \cap B C \subset D_{A}\left(\frac{1}{2}\right)$.

Let $f: \mathbb{R}^{n} \rightarrow \mathbb{R}^{n}$ be $C^{\infty}$. Assume that $f\left(p^{1}\right)=f\left(p^{2}\right)=0$ and there exists $V \in \mathbb{R}$ such that equation (2.4) admits a heteroclinic solution $q(\xi)$ connecting $p^{1}$ to $p^{2}$. Let $X=E(w)$ or $B(w)$ and $A: D_{A} \rightarrow X$ be defined as $A u=u_{\xi \xi}$ as in Lemma 2.6. It can be verified that $f: D_{A} \rightarrow D_{A}$ and $f: X \rightarrow X$ are both $C^{\infty}$ Therefore, $f: D_{A}(\theta) \rightarrow D_{A}(\theta)$ for any $0<\theta<1$ is also $C^{\infty}$.

Consider

$$
\begin{aligned}
u_{\tau} & =u_{\xi \xi}+V u_{\xi}+f(u), \\
u(0) & =\bar{u}_{0}, \bar{u}_{0} \in D_{A}(\theta), \quad 0 \leq \theta \leq 1 .
\end{aligned}
$$

For each $\bar{u}_{0}$, there exists $T>0$ such that a unique classical solution exists in $[0, T]$. Also, $q(\xi)$ is a stationary solution to (2.19). Consider the linear variational equation around $q(\xi)$.

$$
\begin{aligned}
u_{\tau} & =u_{\xi \xi}+V u_{\xi}+D f(q(\xi)) u, \\
u(0) & =\bar{u}_{0}, \bar{u}_{0} \in D_{A}(\theta) .
\end{aligned}
$$

Define $L_{q} u=u_{\xi \xi}+V u_{\xi}+D f(q(\xi)) u$. As a perturbation to $A, L_{q}$ is also a sectorial operator in $X$, cf. [27, page 80$]$.

Definition 2.1. The solution $q$ to equation (2.19) is said to be asymptotically (exponentially) stable modulo spatial shifts if there exists an open set $O \subset D_{A}(\theta), q \in$ $O$, such that for every $\bar{u}_{0} \in O$, there is a constant $c \in \mathbb{R}$, such that

$$
|u(\tau)-q(\cdot+c)|_{D_{A}(\theta)} \leq C e^{-\gamma \tau} .
$$

The zero solution to equation (2.20) is said to be asymptotically stable modulo $q_{\xi}$ if for every $\bar{u}_{0} \in D_{A}(\theta)$, there is a constant $c \in \mathbb{R},\left|u(\tau)-c q_{\xi}\right|_{D_{A}(\theta)} \leq C e^{-\gamma \tau}$.

Lemma 2.8. (Evans) (a) The stationary solution $q$ of (2.19) is asymptotically stable modulo spatial shifts if and only if the zero solution of (2.20) is asymptotically stable modulo $q_{\xi}$.

(b) The zero solution of (2.20) is asymptotically stable modulo $q_{\xi}$ if and only if there exists $\alpha>0$ such that

$$
\sigma\left\{L_{q}\right\} \cap\{\operatorname{Re} \lambda>-\alpha\}=\{0\},
$$

and $\lambda=0$ is a simple eigenvalue with the eigenspace spanned by $q_{\xi}$. 
The following lemma puts a strong restriction on the essential spectrum of $L_{q}$.

Lemma 2.9. (a) Let $M$ be a constant $n \times n$ matrix, $\gamma \in \mathbb{R}$ be the constant in (2.2) and $L u=u_{\xi \xi}+V u_{\xi}+M u$. Then $L$ is a sectorial operator on $X=E_{\mathbb{R}}(w)$, $E_{\mathbb{R}^{ \pm}}(w), B_{\mathbb{R}}(w)$, or $B_{\mathbb{R}^{ \pm}}(w)$ with $D_{L}=E_{\mathbb{R}}^{2}(w), E_{\mathbb{R}^{ \pm}}^{2}(w) \cap B C, B_{\mathbb{R}}^{2}(w)$ or $B_{\mathbb{R}^{ \pm}}^{2}(w) \cap$ $B C$ respectively. Assume furthermore that $\operatorname{Re}(\sigma(M)) \leq-\sigma_{0}<0$ and $\gamma$ satisfy that $\eta_{0}=\left(\gamma^{2}+2|V \gamma|\right) / 4<\sigma_{0}$, then

$$
\operatorname{Re}(\sigma(L)) \leq-\sigma_{0}+\eta_{0}
$$

(b) Let $p^{i}, i=1,2$, satisfy (2.8) and $q(\xi)$ be a heteroclinic solution connecting $p^{1}$ to $p^{2}$. Let $X, \gamma$ and $\eta_{0}$ be as in part (a). Then $\sigma\left\{L_{q}\right\} \cap\left\{\operatorname{Re} \lambda>-\sigma_{0}+\eta_{0}\right\}$ consists of only isolated eigenvalues, each is of finite algebraic multiplicity.

Corollary 2.10. The zero solution $u_{\tau}=u_{\xi \xi}+V u_{\xi}+f_{u}\left(p^{i}\right) u, i=1,2$, is asymptotically stable in the space $X$. The stationary solution $u=p^{i}, i=1,2$, for $u_{\tau}=u_{\xi \xi}+V u_{\xi}+f(u)$ is asymptotically stable.

\section{Stationary solutions that have Layer structures}

The stationary solutions of (1.1) satisfy

$$
\epsilon^{2} u_{x x}+f(u, x, \epsilon)=0 \text {. }
$$

The first set of assumptions are used to construct a stationary solution to system (1.1) that exhibits internal and boundary layers and to obtain the asymptotic expansion of such a solution to any desired order in $\epsilon$.

Assume that there is a partition of $[a, b]$ :

$$
x^{0}=a<x^{1}<\cdots<x^{r}=b .
$$

On each $\left[x^{i-1}, x^{i}\right]$, a $C^{\infty}$ function $p^{i}(x), 1 \leq i \leq r$, is defined with $f_{0}\left(p^{i}(x), x\right)=0$.

H1. $\operatorname{Re} \sigma\left\{f_{0 u}\left(p^{i}(x), x\right)\right\}<0$ for $x^{i-1} \leq x \leq x^{i}, i=1, \cdots, r$.

We introduce a stretched variable $\xi=\left(x-x^{i}\right) / \epsilon$ in a neighborhood of each $x^{i}, 0 \leq i \leq r$. The 0 -th expansion of (3.1) can be written as

$$
u_{\xi \xi}+f_{0}\left(u, x^{i}\right)=0 .
$$

Assume that a $C^{\infty}$ function $q^{i}(\xi)$ is defined for $\xi \in \mathbb{R}$ if $1 \leq i \leq r-1, \xi \in \mathbb{R}^{+}$if $i=0$ and $\xi \in \mathbb{R}^{-}$if $i=r$, such that $u=q^{i}(\xi)$ satisfies (3.2). Also, $q^{i}(\xi) \rightarrow p^{i}\left(x^{i}\right)$ as $\xi \rightarrow-\infty$ for $1 \leq i \leq r$ and $q^{i}(\xi) \rightarrow p^{i+1}\left(x^{i}\right)$ as $\xi \rightarrow \infty$ for $0 \leq i \leq r-1$. Moreover, $q_{\xi}^{i}(0)=0$ for $i=0, r$, cf. (1.2).

The linear homogeneous equation

$$
\phi_{\xi \xi}+f_{0 u}\left(q^{i}(\xi), x^{i}\right) \phi=0
$$

and its adjoint equation

$$
\psi_{\xi \xi}+f_{0 u}^{\tau}\left(q^{i}(\xi), x^{i}\right) \psi=0
$$

are important in our study. (Here $\tau$ denotes the transpose.)

H2. $q_{\xi}^{i}(\xi), \xi \in \mathbb{R}, 1 \leq i \leq r-1$, is the only bounded solution of (3.3) up to constant multiples.

Because of H2, we can show that (3.4) has a unique bounded solution $\psi_{i}(\xi), \xi \in$ $\mathbb{R}, 1 \leq i \leq r-1$, up to constant multiples. See [26]. Moreover, $\psi_{i}$ decays exponentially as $\xi \rightarrow \pm \infty$. 
H3. $\int_{-\infty}^{\infty} \psi_{i}^{\tau}(\xi) f_{0 x}\left(q^{i}(\xi), x^{i}\right) d \xi \neq 0,1 \leq i \leq r-1$.

Hypothesis H3 is expressed by a Melnikov type integral that replaces a hypothesis expressed by potential functions in $[12,13]$. If $n=1,(3.3)$ is self adjoint. Thus $\psi=q_{\xi}^{i}$. The integral in H3 is equal to $D_{x} J\left(x^{i}\right)$ where $J(x)=\int_{p^{i}(x)}^{p^{i+1}(x)} f_{0}(u, x) d u$ as in $[12,13]$.

H4. Let $\phi^{i}(\xi), \xi \in \mathbb{R}^{+}$for $i=0$ and $\xi \in \mathbb{R}^{-}$for $i=r$, be any nontrivial bounded solution for (3.3). Then $\phi_{\xi}^{i}(0) \neq 0$ for $i=0, r$.

We look for the position of the internal layers $x^{i}(\epsilon)=\sum_{j=0}^{\infty} \epsilon^{j} x_{j}^{i}$, formal series solution $\sum_{j=0}^{\infty} \epsilon^{j} u_{j}^{R i}(x)$ to (3.1) in regular layer $\left(x^{i-1}(\epsilon), x^{i}(\epsilon)\right), 1 \leq i \leq r$, and formal series solution $\sum_{j=0}^{\infty} \epsilon^{j} u_{j}^{S i}(\xi), 0 \leq i \leq r$, to the equation

$$
u_{\xi \xi}+f\left(u, x^{i}(\epsilon)+\epsilon \xi, \epsilon\right)=0,
$$

where $\xi=\left(x-x^{i}(\epsilon)\right) / \epsilon$. Let the superscripts " $R$ " and " $S$ " stand for spatial regular and singular layers. Each $u_{j}^{S i}(\xi)$ satisfies a growth condition

$$
\left|u_{j}^{S i}(\xi)\right| \leq C\left(1+|\xi|^{j}\right)
$$

as $|\xi| \rightarrow \infty$ and a boundary condition

$$
u_{j \xi}^{S i}(0)=0
$$

if $i=0, r$. Let the inner expansions of the outer solutions be

$$
\begin{aligned}
& \sum_{j=0}^{\infty} \epsilon^{j} \tilde{u}_{j, 1}^{i}(\xi)=\sum_{j=0}^{\infty} \epsilon^{j} u_{j}^{R, i+1}\left(\epsilon \xi+x^{i}(\epsilon)\right), \\
& \sum_{j=0}^{\infty} \epsilon^{j} \tilde{u}_{j, 2}^{i}(\xi)=\sum_{j=0}^{\infty} \epsilon^{j} u_{j}^{R i}\left(\epsilon \xi+x^{i}(\epsilon)\right) .
\end{aligned}
$$

The right hand sides in the above are formal expressions. Their expansions in $\epsilon$ are well defined even if $x^{i}(\epsilon)$ is a formal series and $u_{j}^{R i}(x)$ is not defined for $x \in \mathbb{R}$. See [22]. Each $\tilde{u}_{j, h}^{i}, h=1,2$, is in fact a polynomial of degree $j$. We say that the solution in the singular layer $\sum \epsilon^{j} u_{j}^{S i}(\xi)$ matches solution $\sum \epsilon^{j} u_{j}^{R i}(x)$ or $\sum \epsilon^{j} u_{j}^{R, i+1}(x)$ if

$$
\begin{aligned}
& \left|u_{j}^{S i}(\xi)-\tilde{u}_{j, 1}^{i}(\xi)\right| \leq C\left(\left(1+|\xi|^{j}\right) e^{\gamma \xi}\right), \quad \xi \leq 0, \\
& \left|u_{j}^{S i}(\xi)-\tilde{u}_{j, 2}^{i}(\xi)\right| \leq C\left(\left(1+|\xi|^{j}\right) e^{-\gamma \xi}\right), \quad \xi \geq 0 .
\end{aligned}
$$

To construct those series solutions, we shall use the result from [22]. We verify that for an equivalent first order system in $\mathbb{R}^{2 n}$, all the conditions in [22] are satisfied. From Lemma 2.2 and H1, $u=p^{i}(x)$ is a hyperbolic equilibrium for the equation

$$
u_{\xi \xi}+f_{0}(u, x)=0
$$

for $x^{i-1} \leq x \leq x^{i}$. That is, $\left(p^{i}(x), 0\right)$ is a hyperbolic equilibrium for the equivalent system

$$
\begin{aligned}
& u_{\xi}=v, \\
& v_{\xi}=-f_{0}(u, x) .
\end{aligned}
$$

Also from Lemma 2.2, the unstable spaces are $n$-dimensional for all $1 \leq i \leq r-1$, and $x$. Thus the hypothesis H1 in [22] is satisfied. 
On the other hand, when $x=x^{i}, 1 \leq i \leq r-1$. Since $q^{i}(\xi) \rightarrow p^{i}\left(x^{i}\right)$ or $p^{i+1}\left(x^{i}\right)$ as $\xi \rightarrow-\infty$ or $+\infty$, based on Lemma 2.2 again, the linearized equation

$$
\begin{aligned}
\phi_{\xi} & =\bar{\phi}, \\
\bar{\phi}_{\xi} & =-f_{0 u}\left(q^{i}(\xi), x^{i}\right) \phi,
\end{aligned}
$$

has exponential dichotomies on $\mathbb{R}^{-}$and $\mathbb{R}^{+}$. From $\mathrm{H} 2,\left(q_{\xi}^{i}(\xi), q_{\xi \xi}^{i}(\xi)\right)$ is the only bounded solution of (3.11) up to a constant factor. From [26], there exists a unique bounded solution $\left(\psi_{i \xi}(\xi), \psi_{i}(\xi)\right)$, up to a constant factor, to the adjoint equation

$$
\begin{aligned}
\psi_{\xi} & =f_{0 u}^{\tau}\left(q_{i}(\xi), x^{i}\right) \bar{\psi}, \\
\bar{\psi}_{\xi} & =-\psi .
\end{aligned}
$$

Cf. (3.3) and (3.4). Denote the right hand side of (3.10) by $F(u, v, x)$. Then

$$
\frac{\partial F}{\partial x}(u, v, x)=\left(\begin{array}{c}
0 \\
-f_{0 x}(u, v)
\end{array}\right) .
$$

It is now clear that $\mathrm{H} 3$ implies [22, $\mathrm{H} 3)]$.

Finally, it is clear that H4 implies that [22, H2)]. The result from [22] yields:

Theorem 3.1. Under the hypotheses $\mathrm{H} 1$ to $\mathrm{H} 4$, there exist unique formal series:

$$
\begin{gathered}
\sum_{j=0}^{\infty} \epsilon^{j} x_{j}^{i}, \quad 0 \leq i \leq r, \quad x_{0}^{i}=x^{i}, x_{j}^{0}=x_{j}^{r}=0 \text { for all } j \geq 1, \\
\sum_{j=0}^{\infty} \epsilon^{j} u_{j}^{R i}(x), \quad u_{0}^{R i}(x)=p^{i}(x), \quad 1 \leq i \leq r,
\end{gathered}
$$

$$
\sum_{j=0}^{\infty} \epsilon^{j} u_{j}^{S i}(\xi), \quad u_{0}^{S i}(\xi)=q^{i}(\xi), \quad \text { with } \begin{cases}\xi \in \mathbb{R}, & 1 \leq i \leq r-1, \\ \xi \in \mathbb{R}^{+}, & i=0, \\ \xi \in \mathbb{R}^{-}, & i=r,\end{cases}
$$

such that (3.14) is the position of the singular layer $x^{i}(\epsilon)$, (3.15) satisfies (3.1), (3.16) satisfies (3.5), (3.6) and (3.7) if $i=0, r$. The functions $u_{j}^{R i}$ and constants $x_{j}^{i}$ are computable by systems of recursive linear algebraic equations. The functions $u_{j}^{\text {Si }}$ are computable by a system of recursive linear nonhomogeneous differential equations. Moreover, the series solutions obtained above satisfy the matching condition (3.8).

\section{EXISTENCE OF WAVE-FRONT-LIKE SOLUTIONS}

4.1. Hypotheses and lemmas. To study time evolution solutions of (1.1), some more hypotheses will be made. Let $L_{q^{i}}: C_{b u} \rightarrow C_{b u}$ be an unbounded operator defined by

$$
L_{q^{i}}(u)(\xi)=u_{\xi \xi}+f_{0 u}\left(q^{i}(\xi), x^{i}\right) u .
$$

The domain $D\left(L_{q^{i}}\right)=C_{b u}^{2}\left(\mathbb{R}, \mathbb{R}^{n}\right)$ for $1 \leq i \leq r-1$ and $D\left(L_{q^{i}}\right)=C_{b u}^{2} \cap B C$, for $i=0, r$.

H5. There exists a constant $\alpha_{0}$ such that all the eigenvalues of $L_{q^{0}}$ and $L_{q^{r}}$ satisfy $\operatorname{Re} \lambda<-\alpha_{0}$. The operator $L_{q^{i}}, 1 \leq i \leq r-1$, has a simple eigenvalue $\lambda=0$ with an eigenvector $q_{\xi}^{i}(\xi)$, all the other spectra satisfy $\operatorname{Re} \lambda<-\alpha_{0}$. 
Hypothesis $\mathrm{H} 5$ ensures the stability of $q^{i}(\xi)$ as a solution to $u_{\tau}=u_{\xi \xi}+f_{0}\left(u, x^{i}\right)$ in $C_{b u}$.

Since $\lambda=0$ is a simple eigenvalue of $L_{q^{i}}, 1 \leq i \leq r-1$, we have

$$
\int_{-\infty}^{\infty} \psi_{i}^{\tau}(\xi) q_{\xi}^{i}(\xi) d \xi \neq 0, \quad 1 \leq i \leq r-1
$$

where $\psi_{i}(\xi)$ is the bounded solution for (3.4). The proof of (4.1) uses Lemma 2.4. If (4.1) were not valid, then $q_{\xi}^{i}$ is in the range of $L_{q^{i}}$. This contradicts the fact $\lambda=0$ is simple. Assume now

H6. $\int_{-\infty}^{\infty} \psi_{i}^{\tau}(\xi) q_{\xi}^{i}(\xi) d \xi \cdot \int_{-\infty}^{\infty} \psi_{i}^{\tau}(\xi) f_{0 x}\left(q^{i}(\xi), x^{i}\right) d \xi>0 \quad$ for $1 \leq i \leq r-1$.

Due to H3 and (4.1), H6 is only a sign condition. We shall see that H6 implies that the position of the wave front near $x^{i}$ moves towards $x^{i}$.

It is useful to note that (4.1) is always valid if $n=1$. In that case, $L_{q^{i}}$ is self adjoint, thus $\psi_{i}(\xi)=q_{\xi}^{i}(\xi)$.

We look for wave front positions that are in open intervals $O^{i}$ containing $x^{i}, 1 \leq$ $i \leq r-1$. We state the following properties of $O^{i}$.

P1. $p^{i}(x)$ and $p^{i+1}(x)$ can be extended smoothly to $O^{i}$ with

$$
\begin{gathered}
f_{0}\left(p^{j}(x), x\right)=0, \\
\operatorname{Re}\left\{\sigma\left(f_{0 u}\left(p^{j}(x), x\right)\right)\right\}<0, \quad j=i, i+1 .
\end{gathered}
$$

P2. There exists a $C^{\infty}$ function $V^{i}: O^{i} \rightarrow \mathbb{R}$ such that for each $x \in O^{i}$, equation

$$
u_{\xi \xi}+V^{i}(x) u_{\xi}+f_{0}(u, x)=0
$$

admits a heteroclinic solution $q^{i}(\xi, x)$, connecting $p^{i}(x)$ to $p^{i+1}(x)$, with $(u(0)-$ $\left.q^{i}(0)\right) \perp q_{\xi}^{i}(0)$. In particular, $V^{i}\left(x^{i}\right)=0$ and $q^{i}\left(\xi, x^{i}\right)=q^{i}(\xi)$. Moreover, $D_{x}^{k} q^{i}(\cdot, x) \in$ $C_{b u}^{j}$ for all $j, k \geq 0$.

P3. The linear equation

$$
\phi_{\xi \xi}+V^{i}(x) \phi_{\xi}+f_{0 u}\left(q^{i}(\xi, x), x\right) \phi=0
$$

has a unique bounded solution $q_{\xi}^{i}(\xi, x)$, up to constant multiples. And the adjoint equation

$$
\psi_{\xi \xi}-V^{i}(x) \psi_{\xi}+f_{0 u}^{\tau}\left(q^{i}(\xi, x), x\right) \psi=0
$$

has a unique bounded solution $\psi_{i}(\xi, x),\left|\psi_{i}(0, x)\right|=1$, up to constant multiples. Furthermore $\psi(., x)$ is a $C^{\infty}$ function of $x$ in the space $C_{b u}$.

P4. In the Banach space $E_{\mathbb{R}}(w), L_{x}^{i} u=u_{\xi \xi}+V^{i}(x) u_{\xi}+f_{o u}\left(q^{i}(\xi, x), x\right) u, 1 \leq i \leq$ $r-1$, has $\lambda=0$ as a simple eigenvalue with eigenvector $q_{\xi}^{i}(\xi, x)$. All the other spectra of $L_{x}^{i}$ satisfy $\operatorname{Re} \lambda<-\alpha_{0}$ for some $\alpha_{0}>0$.

P5. For $x \in O^{i}$, we have

$$
\left\{\int_{-\infty}^{\infty} \psi_{i}^{\tau}(\xi, x) q_{\xi}^{i}(\xi, x) d \xi\right\}\left\{\int_{-\infty}^{\infty} \psi_{i}^{\tau}(\xi, x) f_{0 x}\left(q^{i}(\xi, x), x\right) d \xi\right\}>0, \quad 1 \leq i \leq r-1 .
$$


Also $V^{i}(x)>0(=0,<0)$ if $x<x^{i}\left(=x^{i},>x^{i}\right)$. And

$$
\frac{\partial V^{i}\left(x^{i}\right)}{\partial x}=\frac{-\int_{-\infty}^{\infty} \psi_{i}^{\tau}(\xi) f_{0 x}\left(q^{i}(\xi), x^{i}\right) d \xi}{\int_{-\infty}^{\infty} \psi_{i}^{\tau}(\xi) q_{\xi}^{i}(\xi) d \xi}<0
$$

Lemma 4.1. Assume that $\mathrm{H} 1, \mathrm{H} 2$ and $\mathrm{H} 5$ are satisfied. Then there exist open intervals $O^{i}$ containing $x^{i}, 1 \leq i \leq r-1$ such that Properties $\mathrm{P} 1-\mathrm{P} 4$ hold. Moreover, if $\mathrm{H} 3$ and $\mathrm{H} 6$ are also satisfied, then Property $\mathrm{P} 5$ also holds.

The proof of Lemma 4.1 will be given in $\S 7$.

Consider the nonhomogeneous equation, $1 \leq i \leq r-1$,:

$$
u_{\xi \xi}+V^{i}(x) u_{\xi}+f_{0 u}\left(q^{i}(\xi, x), x\right) u+V_{1} q_{\xi}^{i}(\xi, x)=g(\xi) .
$$

Here $V^{i}(x)$ and $q^{i}(\xi, x)$ are the functions in $\mathrm{P} 3, V_{1} \in \mathbb{R}$ is a parameter. Suppose that $g \in E_{\mathbb{R}}^{m}\left(1+|\xi|^{k}\right), m, k \geq 0$.

Lemma 4.2. Assume that $\mathrm{H} 1, \mathrm{H} 2$ and $\mathrm{H} 5$ are satisfied. Let $1 \leq i \leq r-1$. Then there exists a unique $C^{\infty}$ function $V_{*}^{i}: O^{i} \times E_{\mathbb{R}}^{m}\left(1+|\xi|^{k}\right) \rightarrow \mathbb{R}$ such that if $V_{1}=$ $V_{*}^{i}(x, g)$, then there is a unique solution $u(\xi, x, g)$ of $(4.8)$ with $u \in E_{\mathbb{R}}^{m+2}\left(1+|\xi|^{k}\right)$ and $u(0) \perp q_{\xi}^{i}(0, x)$. Moreover, $u(\cdot, x, g)$ is $C^{\infty}$ in $(x, g)$ with respect to the indicated norms.

In the rest of this section, we always assume P1-P4. Sometime P5 is also assumed as will be indicated in the text.

4.2. Formal power series solutions in $(R R)^{i}$. Let the position of the $i$-th internal layer be

$$
\eta^{i}(t, \epsilon)=\sum_{j=0}^{\infty} \epsilon^{j} \eta_{j}^{i}(t), \quad 1 \leq i \leq r-1 .
$$

For convenience, let $\eta^{0}(t, \epsilon)=a, \eta^{r}(t, \epsilon)=b$.

Assume that $\eta_{0}^{i}(t) \in O^{i}$ and $\eta_{0}^{i-1}(t)<\eta_{0}^{i}(t)$. In the interval $\left(\eta_{0}^{i-1}(t), \eta_{0}^{i}(t)\right)$, $1 \leq i \leq r$, we seek formal series solution

$$
u^{R R i}(x, t, \epsilon)=\sum_{j=0}^{\infty} \epsilon^{j} u_{j}^{R R i}(x)
$$

that satisfies (1.1). Since $f$ does not depend on $t$, by induction, we can show that $u_{j}^{R R i}$ does not depend on $t$. Expanding in powers of $\epsilon$, we have (drop the superscripts):

$$
\begin{gathered}
0=f_{0}\left(u_{0}(x), x\right) \\
0=f_{0 u}\left(u_{0}(x), x\right) u_{1}+f_{1}\left(u_{0}(x), x\right), \\
\ldots \\
0=f_{0 u}\left(u_{0}(x), x\right) u_{k}+u_{k-2, x x}+\sum_{\alpha, \delta} C_{\alpha \delta} D_{u}^{|\alpha|} f_{\delta}\left(u_{0}(x), x\right) u^{\alpha} .
\end{gathered}
$$

Here $\alpha=\left(\alpha_{1}, \ldots, \alpha_{k-1}\right)$ is a multi-index, $u^{\alpha}=u_{1}^{\alpha_{1}} \ldots u_{k-1}^{\alpha_{k-1}}, \sum_{j=1}^{k-1} j \alpha_{j}+\delta=k, C_{\alpha \delta}$ is a constant. 
Let the solution of (4.10) be $u_{0}(x)=p^{i}(x), 1 \leq i \leq r$. Since $O^{i}$ satisfies P1, then $f_{0 u}\left(u_{0}(x), x\right)$ is nonsingular. Thus, $u_{j}^{R R i}, j \geq 1$, can be solved successively from system (4.10)- $\left(4.10_{k}\right), k \geq 1$.

Theorem 4.3. Assume that $O^{i}$ satisfies $\mathrm{P} 1$. Let $\eta_{0}^{i}(t) \in O^{i}$ and $\eta_{0}^{i-1}(t)<\eta_{0}^{i}(t)$ for $1 \leq i \leq r-1$. Then there exists a unique formal series solution $u^{R R i}(x, t, \epsilon)=$ $\sum_{j=0}^{\infty} \epsilon^{j} u_{j}^{R R i}(x)$ to

$$
0=\epsilon^{2} u_{x x}+f(u, x, \epsilon), \quad \eta_{0}^{i-1}(t)<x<\eta_{0}^{i}(t) .
$$

With $u_{0}^{i}=p^{i}(x)$, it can be obtained from system (4.10)-(4.10k), $k \geq 1$, recursively.

4.3. Formal solutions in $(S R)^{i}$. First consider the internal layers, $1 \leq i \leq r-1$. Let the position of the internal layer, at $t=0$, be $\eta^{i}(0, \epsilon)=\sum_{j=0}^{\infty} \epsilon^{j} \bar{\eta}_{j}^{i}$, i.e., $\eta_{j}^{i}(0)=\bar{\eta}_{j}^{i}$. Assume in this section that $\left\{\bar{\eta}_{j}^{i}\right\}_{0}^{\infty}$ is given. The problem of determining $\left\{\bar{\eta}_{j}^{i}\right\}_{0}^{\infty}$ will be discussed in $\S 5$. Assume that

$$
\bar{\eta}_{0}^{i} \in O^{i}, 1 \leq i \leq r-1 \quad \text { and } \quad \bar{\eta}_{0}^{i-1}<\bar{\eta}_{0}^{i}, 2 \leq i \leq r-1 .
$$

Let $\xi=\left[x-\eta^{i}(t, \epsilon)\right] / \epsilon$. We seek the layer position $\eta^{i}(t, \epsilon)$ and the formal solution $u^{S R i}(\xi, t, \epsilon)$ near the singular layer at $x=\eta^{i}(t, \epsilon)$. Since

$$
u(x, t, \epsilon)=u^{S R i}\left(\left(x-\eta^{i}(t, \epsilon)\right) / \epsilon, t, \epsilon\right),
$$

from (1.1), $u^{S R i}(\xi, t, \epsilon)$ satisfies (drop the super-indices):

$$
\epsilon u_{t}=u_{\xi \xi}+D_{t} \eta(t, \epsilon) u_{\xi}+f(u, \epsilon \xi+\eta(t, \epsilon), \epsilon) .
$$

Let $u(\xi, t, \epsilon)=\sum_{j=0}^{\infty} \epsilon^{j} u_{j}(\xi, t), \eta(t, \epsilon)=\sum_{j=0}^{\infty} \epsilon^{j} \eta_{j}(t)$. Expanding in powers of $\epsilon$, we have $\left(\eta^{\prime}(t)\right.$ denotes $\left.\frac{d}{d t} \eta(t)\right)$ :

$$
\begin{gathered}
0=\eta_{0}^{\prime}(t) u_{0 \xi}+u_{0 \xi \xi}+f_{0}\left(u_{0}, \eta_{0}(t)\right), \\
u_{0 t}=\eta_{1}^{\prime}(t) u_{0 \xi}+f_{0 x}\left(u_{0}, \eta_{0}(t)\right) \eta_{1}(t) \\
+u_{1 \xi \xi}+\eta_{0}^{\prime}(t) u_{1 \xi}+f_{0 u}\left(u_{0}, \eta_{0}(t)\right) u_{1} \\
+\left\{f_{0 x}\left(u_{0}, \eta_{0}(t)\right) \xi+f_{1}\left(u_{0}, \eta_{0}(t)\right)\right\} \\
\ldots \\
u_{k-1, t}=\eta_{k}^{\prime}(t) u_{0 \xi}+f_{0 x}\left(u_{0}, \eta_{0}(t)\right) \eta_{k}(t) \\
+u_{k \xi \xi}+\eta_{0}^{\prime}(t) u_{k \xi}+f_{0 u}\left(u_{0}, \eta_{0}(t)\right) u_{k} \\
+\sum_{j=1}^{k-1} \eta_{j}^{\prime}(t) u_{k-j, \xi}+\sum C_{\alpha \beta \gamma \delta} D_{u}^{|\alpha|} D_{x}^{|\beta|+\gamma} f_{\delta}\left(u_{0}, \eta_{0}(t)\right) u^{\alpha} \eta^{\beta} \xi^{\gamma} .
\end{gathered}
$$

Here $\alpha=\left(\alpha_{1}, \ldots, \alpha_{k-1}\right), \beta=\left(\beta_{1}, \ldots, \beta_{k-1}\right)$ are multi-indices, $\delta$ and $\gamma$ are nonnegative integers, $u^{\alpha}=u_{1}^{\alpha_{1}} \ldots u_{k-1}^{\alpha_{k-1}}, \eta^{\beta}=\eta_{1}^{\beta_{1}} \ldots \eta_{k-1}^{\beta_{k-1}}, \delta+\gamma+\sum_{j=1}^{k-1}\left(\alpha_{j}+\beta_{j}\right) \cdot j=$ $k, C_{\alpha \beta \gamma \delta}$ is a constant.

From P2, there exists a unique heteroclinic solution

$$
u_{0}(\xi, t)=q^{i}\left(\xi, \eta_{0}(t)\right)
$$


to (4.13) connecting $p^{i}\left(\eta_{0}^{i}(t)\right)$ to $p^{i+1}\left(\eta_{0}^{i}(t)\right)$ with $\left(u_{0}(0, t)-q^{i}(0)\right) \perp q_{\xi}^{i}(0)$, provided that

$$
\eta_{0}^{\prime}(t)=V^{i}\left(\eta_{0}(t)\right) .
$$

With the initial condition $\eta_{0}^{i}(t)=\bar{\eta}_{0}^{i}$, (4.15) uniquely determines $\eta_{0}^{i}(t)$ as long as $\eta_{0}^{i} \in O^{i}$. From (4.14) and the last assertion of P2, we see that $D_{t}^{j} u_{0} \in C_{b u}^{m}$ for all $j, m \geq 0$.

If we also assume P5, then $x=x^{i}$ is a stable equilibrium of (4.15) in $O^{i}$. Since $\bar{\eta}_{0}^{i} \in O^{i}$, we have $\eta_{0}^{i}(t) \in O^{i}$ for all $t \geq 0$ and approaches $x^{i}$ as $t \rightarrow \infty$.

We compute the sequences $\left\{u_{j}\right\}_{j=0}^{\infty}$ and $\left\{\eta_{j}\right\}_{j=0}^{\infty}$ by induction. Assume that $u_{j}$ and $\eta_{j}, 0 \leq j \leq k-1$, have been obtained, $u_{j}$ is written as

$$
u_{j}(\xi, t)=U_{j}\left(\xi, x, x_{1}, \ldots, x_{j}\right)
$$

where $x=\eta_{0}(t), x_{1}=\eta_{1}(t), \ldots, x_{k-1}=\eta_{k-1}(t)$, and $D_{y}^{\alpha} U_{j} \in E^{m}\left(1+|\xi|^{j}\right)$ where $y=\left(x, x_{1}, \ldots, x_{j}\right), \alpha=\left(\alpha_{0}, \alpha_{1}, \ldots, \alpha_{j-1}\right), m \geq 0$, is an arbitrary integer. Also assume that $\eta_{j}^{\prime}(t)=V_{j}^{i}\left(x, x_{1}, \ldots, x_{j}\right)$ and $\eta_{j}(t) \rightarrow x_{j}^{i}$ as $t \rightarrow \infty$. Let $\eta_{k}(t)=x_{k}$ and $\eta_{k}^{\prime}(t)=V_{k}$. We can write $u_{k-1, t}=\sum_{\ell=0}^{k-1} \frac{\partial U_{k-1}}{\partial x_{\ell}} \cdot V_{\ell}^{i}\left(x, \ldots, x_{\ell}\right)$ where $x_{0}=x$ and $V_{0}^{i}(x)=V^{i}(x)$. Equation $\left(4 \cdot 13_{k}\right)$ can be written as

$$
\begin{aligned}
u_{k \xi \xi} & +V^{i}(x) u_{k \xi}+f_{\text {ou }}\left(q^{i}(\xi, x), x\right) u_{k}+V_{k} q_{\xi}^{i}(\xi, x) \\
& =h_{k}\left(\xi, U_{0}, U_{1}, \ldots, U_{k-1}, x, x_{1}, \ldots, x_{k}\right),
\end{aligned}
$$

where

$$
\begin{aligned}
h_{k}= & f_{0}\left(U_{0}, x\right) x_{k}+\sum_{j=1}^{k-1} V_{j}^{i}\left(x, \ldots, x_{j}\right) U_{k-j, \xi} \\
& +\sum C_{\alpha \beta \gamma \delta} D_{u}^{|\alpha|} D_{x}^{|\beta|+\gamma} f_{\delta}\left(U_{0}, x\right) U^{\alpha} \eta^{\beta} \xi^{\gamma}-\sum_{\ell=0}^{k-1} \frac{\partial U_{k-1}}{\partial x_{\ell}} V_{\ell}^{i}\left(x, \ldots, x_{\ell}\right), \\
& \quad \text { and } \eta^{\beta}=x_{1}^{\beta_{1}}, \ldots, x_{k-1}^{\beta_{k-1}} .
\end{aligned}
$$

From the induction assumptions, we can verify that $D_{y}^{\alpha} h_{k} \in E^{m}\left(1+|\xi|^{k}\right)$ for all $m \geq 0$, where $\alpha=\left(\alpha_{0}, \ldots, \alpha_{k}\right)$ and $y=\left(x, \ldots, x_{k}\right)$. Therefore by Lemma 4.2 , there exists a unique $C^{\infty}$ function $V_{k}^{i}: O^{i} \times \mathbb{R}^{k} \rightarrow \mathbb{R}$ such that if $V_{k}=V_{k}^{i}\left(x, x_{1}, \ldots, x_{k}\right)$, then (4.16) has a unique solution $U_{k}\left(\xi, x, x_{1}, \ldots, x_{k}\right), U_{k}\left(0, x, \ldots, x_{k}\right) \perp q_{\xi}^{i}(0, x)$ and

$$
D_{y}^{\alpha} U_{k} \in E^{m}\left(1+|\xi|^{k}\right)
$$

for all $m \geq 0$. With the initial condition $x_{k}(0)=\bar{\eta}_{k}^{i}$, equation

$$
x_{k}^{\prime}=V_{k}^{i}\left(x, x_{1}, \ldots, x_{k}\right)
$$

has a unique solution $x_{k}=\eta_{k}(t)$. Let

$$
u_{k}(\xi, t)=U_{k}\left(\xi, \eta_{0}(t), \ldots, \eta_{k}(t)\right) .
$$

This is clearly a solution to $\left(4.13_{k}\right)$ and satisfies

$$
\left|D_{t}^{\ell} u_{j}^{i}\right| \in E^{m}\left(1+|\xi|^{j}\right), \quad \ell, m \geq 0 .
$$

Since $h_{k}$ is linear in $x_{k}$ with $\frac{\partial h_{k}}{\partial x_{k}}=-f_{0 x}\left(u_{0}, x\right)$, and $\left\{h_{k}-V_{k} q_{\xi}^{i}(\xi, x)\right\} \in\left\{\psi_{i}(\cdot, x)\right\}^{\perp}$, see the proof of Lemma 4.2, thus $V_{k}^{i}$ is a linear function of $x_{k}$. 
If $\mathrm{P} 5$ is also assumed, then

$$
\frac{\partial V_{k}^{i}}{\partial x_{k}}=-\left\{\int_{-\infty}^{\infty} \psi_{i}^{\tau}(\xi, x) q_{\xi}^{i}(\xi, x) d \xi\right\}^{-1} \cdot\left\{\int_{-\infty}^{\infty} \psi_{i}^{\tau}(\xi, x) f_{0 x}\left(q^{i}(\xi, x), x\right) d \xi\right\}<0 .
$$

Recall that in this case, $\eta_{j}(t) \rightarrow x_{j}^{i}, 0 \leq j \leq k-1$ as $t \rightarrow \infty$. Also, when $x_{j}=$ $x_{j}^{i}, 0 \leq j \leq k-1, x_{k}=x_{k}^{i}$ is a stable equilibrium solution to (4.17) and $\eta_{k}(t) \rightarrow x_{k}^{i}$ as $t \rightarrow \infty$. See Theorem 3.1 for $\left\{x_{j}^{i}\right\}_{j=0}^{k}$. Finally $u_{k}(\xi, t) \rightarrow U_{k}\left(\xi, x_{0}^{i}, \ldots, x_{k}^{i}\right)$ as $t \rightarrow \infty$, the latter is $u_{k}^{S i}(\xi)$ in Theorem 3.1 .

Next, consider the boundary layers, $i=0, r$. At the boundary layer $(S R)^{0}$ near $x=a$, let $x=a+\epsilon \xi, u(x, t, \epsilon)=u^{S R 0}(\xi, t, \epsilon)=\sum_{j=0}^{\infty} \epsilon^{j} u_{j}^{S R 0}(\xi, t)$. The system for $\left\{u_{j}^{S R 0}\right\}$ is simpler than $(4.13)-\left(4.13_{k}\right)$ since the layer position does not move. After dropping the super-indices, we have

$$
\begin{gathered}
0=u_{0 \xi \xi}+f_{0}\left(u_{0}, a\right), \\
u_{0 t}=u_{1 \xi \xi}+f_{0 u}\left(u_{0}, a\right) u_{1}+f_{0 x}\left(u_{0}, a\right) \xi+f_{1}\left(u_{0}, a\right), \\
u_{k-1, t}=u_{k \xi \xi}+f_{0 u}\left(u_{0}, a\right) u_{k}+\sum C_{\alpha \gamma \delta} D_{u}^{|\alpha|} D_{x}^{\gamma} f_{\delta}\left(u_{0}, a\right) u^{\alpha} \xi^{\gamma} .
\end{gathered}
$$

Here $\alpha=\left(\alpha_{1}, \ldots, \alpha_{k-1}\right), u^{\alpha}=u_{1}^{\alpha_{1}} \ldots u_{k-1}^{\alpha_{k-1}}, \gamma \geq 0, \delta+\gamma+\sum_{j=1}^{k-1} j \alpha_{j}=k$ and $C_{\alpha \gamma \delta}$ is a constant. The boundary conditions

$$
u_{j \xi}(0)=0
$$

are imposed on $\left\{u_{j}\right\}_{j=0}^{\infty}$.

Since $q^{0}(\xi)$ satisfies $(4.20)$ and $(4.21)$, set $u_{0}(\xi, t)=q^{0}(\xi) \cdot u_{0} \in C_{b u}^{m}\left(\mathbb{R}^{+}, \mathbb{R}^{n}\right)$ for all $m \geq 0$. Observe that the right hand side of $\left(4.20_{j}\right)$ does not depend on $t$. Thus, $u_{j t}=0$ for all $j \geq 0$. After rewriting $\left(4.20_{j}\right)$ and $(4.21)$ to a first order system in $\mathbb{R}^{2 n}$, we find they correspond to $(5.10 \mathrm{j})$ in $[22, \S 5]$. We look for a solution satisfying

$$
u_{j} \in E_{\mathbb{R}^{+}}^{m}\left(1+|\xi|^{j}\right) .
$$

This condition correspond to (5.11j) in [22]. Moreover Hypothesis H4 implies assumption $\mathrm{H} 2$ in [22]. From the results of [22], we conclude that there exists $\left\{u_{j}^{S R 0}\right\}_{j=0}^{\infty}$ satisfying $(4.20)-\left(4.20_{j}\right), j \geq 1$, and (4.21) and the growth condition (4.22). In fact $u_{j}^{S R 0}(\xi, t)=u_{j}^{S i}(\xi)$ as in Theorem 3.1 of this paper. Similar arguments also apply to $(S R)^{r}$.

Theorem 4.4. (I) Assume $\mathrm{P} 1-\mathrm{P} 4$ and $\sum_{j=0}^{\infty} \epsilon^{j} \bar{\eta}_{j}^{i}$ is given that satisfies (4.11), $1 \leq i \leq r-1$, and $\sum \epsilon^{j} \bar{\eta}_{j}^{0}=a, \sum \epsilon^{j} \bar{\eta}_{j}^{r}=b$. Let $\bar{t}>0$ be such that $\eta_{0}^{i} \in O^{i}$ for $0 \leq t<\bar{t}$. Then there exist unique formal series for $0 \leq t<\bar{t}$,

$$
\eta^{i}(t, \epsilon)=\sum \epsilon^{j} \eta_{j}^{i}(t), 0 \leq i \leq r, \quad \eta_{j}^{i}(0)=\bar{\eta}_{j}^{i},
$$

$$
u^{S R i}(\xi, t, \epsilon)=\sum \epsilon^{j} u_{j}^{S R i}(\xi, t), 0 \leq i \leq r, \quad u_{0}^{S R i}(\xi, t)=q^{i}\left(\xi, \eta_{0}^{i}(t)\right),
$$


with $u_{j}^{S R i}$ defined for $\xi \in \mathbb{R}$ if $1 \leq i \leq r-1, \xi \in \mathbb{R}^{+}$if $i=0, \xi \in \mathbb{R}^{-}$if $i=r$ such that the followings are satisfied: (4.23) and (4.24) formally satisfy (4.12). $u_{j}^{S R i}(\xi, t)$ satisfies (4.19), and the boundary condition (4.21) if $i=0, r$.

The series $\sum \epsilon^{j} u_{j}^{S R i}$ is computable recursively from (4.13)-(4.13k), $k \geq 1$, and $\sum \epsilon^{j} \eta_{j}^{i}(t)$ is computable recursively from (4.15) if $j=0$ or (4.17) if $j \geq 1$.

(II) If $\mathrm{P} 5$ is also assumed then $\bar{t}=\infty$. Moreover, $\eta_{j}^{i}(t) \rightarrow x_{j}^{i}$ as $t \rightarrow \infty$, where $\sum \epsilon^{j} x_{j}^{i}$ is the stationary front as in Theorem 3.1. Also $u_{j}^{S R i}(\xi, t) \rightarrow u_{j}^{S i}(\xi)$ as $t \rightarrow \infty$, where $\sum \epsilon^{j} u_{j}^{S i}(\xi)$ is the formal series solution in the stationary singular layer as in Theorem 3.1.

Corollary 4.5. Assume $\mathrm{H} 1, \mathrm{H} 2, \mathrm{H} 4$ and $\mathrm{H} 5$, then the results in Part (I) of Theorem 4.4 are valid. If furthermore, $\mathrm{H} 3$ and $\mathrm{H} 6$ are also assumed, then results in Part (II) of that theorem are also valid.

Remark. From Corollary 4.5, Hypotheses H3 and H6 imply that the stationary wave-front-like solution in Theorem 3.1 is exponentially stable. If H6 is replaced by

$$
\int_{-\infty}^{\infty} \psi_{i}^{\tau}(\xi) q_{\xi}^{i}(\xi) d \xi \cdot \int_{-\infty}^{\infty} \psi_{i}^{\tau}(\xi) f_{0 x}\left(q^{i}(\xi), x^{i}\right) d \xi>0 \quad \text { for one of } 1 \leq i \leq r-1,
$$

then $\frac{\partial V^{i}\left(x^{i}\right)}{\partial x}>0$ and the solution is unstable.

\section{Solutions in the initial LAyer}

In the initial layer near $t=0$, we use the stretched time $\tau=t / \epsilon$. (1.1) is now

$$
u_{\tau}=\epsilon^{2} u_{x x}+f(u, x, \epsilon) .
$$

5.1. Assumptions on the initial conditions. We assume that the initial data $\bar{u}(x, \epsilon)$ has a layer structure described as follows. There is a partition of the interval $[a, b]$

$$
a=\bar{\eta}^{0}<\bar{\eta}^{1}<\ldots<\bar{\eta}^{r}=b
$$

with $\bar{\eta}^{i} \in O^{i}, 1 \leq i \leq r-1$. At each $\bar{\eta}^{i}, 1 \leq i \leq r-1$, using the stretched variable $\xi=\left(x-\bar{\eta}^{i}\right) / \epsilon$, we have $\bar{u}(x, \epsilon)=\bar{u}\left(\bar{\eta}^{i}+\epsilon \xi, \epsilon\right)=\overline{\bar{u}}^{S i}(\xi, \epsilon),\left(a-\bar{\eta}^{i}\right) / \epsilon \leq \xi \leq\left(b-\bar{\eta}^{i}\right) / \epsilon$. Assume that

H7.

$$
\begin{gathered}
\bar{u}(x, \epsilon)=\sum_{j=0}^{\infty} \epsilon^{j} \bar{u}_{j}^{R i}(x), \quad \bar{\eta}^{i-1}<x<\bar{\eta}^{i}, 1 \leq i \leq r . \\
\overline{\bar{u}}^{S i}(\xi, \epsilon)=\sum_{j=0}^{\infty} \epsilon^{j} \overline{\bar{u}}_{j}^{S i}(\xi), \quad 0 \leq i \leq r . \\
\overline{\bar{u}}_{j \xi}^{S i}(0)=0, \quad j \geq 0, i=0, r .
\end{gathered}
$$

The functions $\bar{u}_{j}^{R i}$ is $C^{\infty}$ and has a $C^{\infty}$ extension to $\left[\bar{\eta}^{i-1}, \bar{\eta}^{i}\right]$.

Observe that $\overline{\bar{u}}^{S i}(\xi, \epsilon)$ is defined for $\xi$ in an interval of size $O\left(\epsilon^{-1}\right)$. As the result of a formal expansion, assume that $\overline{\bar{u}}_{j}^{S i}$ is $C^{\infty}$, and is defined for $\xi \in \mathbb{R}, \mathbb{R}^{+}$or $\mathbb{R}^{-}$ if $1 \leq i \leq r-1, i=0$ or $i=r$. For example, $\overline{\bar{u}}_{0}^{S i}(\xi)$ is the limit of $\overline{\bar{u}}^{S i}(\xi, \epsilon)$ as $\epsilon \rightarrow 0$, uniformly in any compact subset of $\xi$. 
Let the inner expansion of the outer power series be

$$
\begin{aligned}
& \sum_{j=0}^{\infty} \epsilon^{j} \tilde{\tilde{u}}_{j, 1}^{R i}(\xi)=\sum_{j=0}^{\infty} \epsilon^{j} \bar{u}_{j}^{R i}\left(\bar{\eta}^{i}+\epsilon \xi\right), \quad 1 \leq i \leq r \\
& \sum_{j=0}^{\infty} \epsilon^{j} \tilde{\tilde{u}}_{j, 2}^{R i}(\xi)=\sum_{j=0}^{\infty} \epsilon^{j} \bar{u}_{j}^{R(i+1)}\left(\bar{\eta}^{i}+\epsilon \xi\right), \quad 0 \leq i \leq r-1
\end{aligned}
$$

Here, $\tilde{\tilde{u}}_{j, \nu}^{R i}(\xi), \nu=1,2$, is a polynomial of degree $j$. Observe to perform the formal expansion, the right hand sides only have to be defined in a neighborhood of zero.

H8. Each $\overline{\bar{u}}_{j}^{S i} \in E^{m}\left(1+|\xi|^{j}\right)$, for all $m \geq 0$, and the matching conditions with outer expansions are satisfied:

$$
\begin{aligned}
& \overline{\bar{u}}_{j}^{S i}(\xi)-\tilde{\tilde{u}}_{j, 1}^{R i}(\xi) \in E_{\mathbb{R}^{-}}^{m}\left(\left(1+|\xi|^{j}\right) e^{-\gamma|\xi|}\right), \\
& \overline{\bar{u}}_{j}^{S i}(\xi)-\tilde{\tilde{u}}_{j, 2}^{R i}(\xi) \in E_{\mathbb{R}^{+}}^{m}\left(\left(1+\xi^{j}\right) e^{-\gamma \xi}\right),
\end{aligned}
$$

where $\gamma>0$ is a constant.

General discussion of matching conditions can be found in $[6,7]$.

In the regular region, the 0 -th order equation of (5.1) is

$$
u_{\tau}=f_{0}(u, x), \quad x \in\left[\bar{\eta}^{i-1}, \bar{\eta}^{i}\right] .
$$

Let $V^{i}$ be the function in $\mathrm{P} 2$, (ii) so that the following equation

$$
u_{\tau}=u_{\xi \xi}+V^{i}\left(\bar{\eta}^{i}\right) u_{\xi}+f_{0}\left(u, \bar{\eta}^{i}\right)
$$

has a stationary solution (heteroclinic solution, $\left.u_{\tau}=0\right) q^{i}\left(\xi, \bar{\eta}^{i}\right)$ connecting $p^{i}\left(\bar{\eta}^{i}\right)$ to $p^{i+1}\left(\bar{\eta}^{i}\right), 1 \leq i \leq r-1$. For $i=0, r$, let $q^{i}\left(\xi, \bar{\eta}^{i}\right)=q^{i}(\xi), V^{i}\left(\bar{\eta}^{i}\right)=0$. Recall that $q^{0}(\xi), \xi \geq 0$, and $q^{r}(\xi), \xi \leq 0$, are stable stationary solutions of (5.6) satisfying boundary conditions at $x=a$ or $x=b$, approaching $p^{1}(a)$ as $\xi \rightarrow+\infty$ or $p^{r}(b)$ as $\xi \rightarrow-\infty$ respectively.

According to Lemma 2.6, $D_{\xi}^{2}$ is a sectorial operator in $X=\mathbb{B}_{\mathbb{R}}(w)$, so is its perturbation $A u=u_{\xi \xi}+V^{i}\left(\bar{\eta}^{i}\right) u_{\xi}, 1 \leq i \leq r-1$. When $i=0, r, A$ is sectorial in $X=B_{\mathbb{R}^{ \pm}}(w)$ with $D_{A}=B_{\mathbb{R}^{ \pm}}^{2}(w) \cap(B C)$. Observe that $u \rightarrow f_{0}\left(u, \bar{\eta}^{i}\right)$ maps $D_{A}$ to itself. Let $u(0)=u_{0} \in D_{A}$. Local existence for solutions of (5.6) in $B_{\mathbb{R}}(w), 1 \leq i \leq$ $r-1$, or $B_{\mathbb{R}^{ \pm}}(w), i=0, r$, has been established. In particular, $u \in$ $C^{1}\left(\left[0, t_{0}\right]: X\right) \cap C\left(\left[0, t_{0}\right]: D_{A}\right)$. See $[5]$

H9. (i) For each $x \in\left[\bar{\eta}^{i-1}, \bar{\eta}^{i}\right]$, the equilibrium $p^{i}(x)$ of (5.5) attracts $\bar{u}_{0}^{i}(x)$. (ii) The stationary solution $q^{i}(\xi)$ of (5.6) attracts $\overline{\bar{u}}_{0}^{S i}(\xi)$ in the space $B_{\mathbb{R}^{+}}^{2}(w)$ or $B_{\mathbb{R}^{-}}^{2}(w)$ if $i=0, r$; the stationary solution $q^{i}\left(\xi, \bar{\eta}^{i}\right)$ of $(5.6)$ attracts $\overline{\bar{u}}_{0}^{S i}(\xi)$ in the space $B_{\mathbb{R}}^{2}(w)$ modulo a spatial shift if $1 \leq i \leq r-1$.

H9, (i) is a reasonable assumption since $\mathrm{H} 1$ and $\mathrm{P} 1$ imply that $p^{i}(x)$ is a stable solution for (5.5). H9, (ii) is also a reasonable assumption since from $\mathrm{H} 8$, we have $\overline{\bar{u}}_{0}^{S i} \in B^{2}(w)$ and we can prove the following lemma.

Lemma 5.1. $q^{0}\left(\xi, \bar{\eta}^{0}\right)$ and $q^{r}\left(\xi, \bar{\eta}^{r}\right)$, are asymptotically stable stationary solutions in $B_{\mathbb{R}^{+}}^{2}(w)$ and $B_{\mathbb{R}^{-}}^{2}(w)$ respectively. $q^{i}\left(\xi, \bar{\eta}^{i}\right), 1 \leq i \leq r-1$, are asymptotically stable in $B_{\mathbb{R}}^{2}(w)$ modulo spatial shifts.

For $x \in O^{i}$, define

$$
L_{q^{i}} u=u_{\xi \xi}+V^{i}(x) u_{\xi}+f_{0 u}\left(q^{i}(\xi, x), x\right) u, \quad 0 \leq i \leq r .
$$


See P2 for $V^{i}(x)$ and $q^{i}(\xi, x) . L_{q^{i}}$ is a closed linear operator if $X=E_{\mathbb{R}}(w), 1 \leq$ $i \leq r-1$, with $D\left(L_{q^{i}}\right)=E_{\mathbb{R}}^{2}(w)$; or if $X=E_{\mathbb{R}^{ \pm}}(w), i=0$ or $r$, with $D\left(L_{q^{i}}\right)=$ $E_{\mathbb{R}^{ \pm}}^{2}(w) \cap(B C)$. Consider

$$
u_{\tau}=L_{q^{i}} u, \quad 0 \leq i \leq r .
$$

Also the boundary condition $u_{\xi}(0)=0$ is imposed if $i=0, r$.

Lemma 5.2. Equation (5.7) is asymptotically stable in $E_{\mathbb{R}^{ \pm}}^{2}(w)$ if $i=0, r$. It is asymptotically stable modulo $q_{\xi}^{i}(\cdot, x)$ if $1 \leq i \leq r-1$.

\subsection{Formal power series solutions in $(R S)^{i}$. Let}

$$
u^{R S i}(x, \tau, \epsilon)=\sum_{j=0}^{\infty} \epsilon^{j} u_{j}^{R S i}(x, \tau) .
$$

From (5.1), expanding in powers of $\epsilon$ and dropping the super indices, we have for $x \in\left[\bar{\eta}^{i-1}, \bar{\eta}^{i}\right]$,

$$
\begin{aligned}
u_{0 \tau} & =f_{0}\left(u_{0}, x\right), \\
u_{1 \tau} & =f_{0 u}\left(u_{0}, x\right) u_{1}+f_{1}\left(u_{0}, x\right), \\
u_{2 \tau} & =u_{0 x x}+f_{0 u}\left(u_{0}, x\right) u_{2}+f_{0 u u} \cdot u_{1}^{2} / 2+f_{1 u} \cdot u_{1}+f_{2}, \\
& \ldots \\
u_{k \tau} & =u_{k-2, x x}+f_{0 u}\left(u_{0}, x\right) u_{k}+\sum C_{\alpha \delta} D_{u}^{|\alpha|} f_{\delta} \cdot u^{\alpha} .
\end{aligned}
$$

Here $\delta \geq 0, \alpha=\left(\alpha_{1}, \ldots, \alpha_{k-1}\right), u^{\alpha}=u_{1}^{\alpha_{1}} \ldots u_{k-1}^{\alpha_{k-1}}, \delta+\sum j \alpha_{j}=k, C_{\alpha \delta}$ is a constant.

With $x$ as a parameter, $(5.8)-\left(5.8_{k}\right), k \geq 1$, are to be solved recursively with the initial data

$$
u_{j}(x, 0)=\bar{u}_{j}(x), \quad j \geq 0,
$$

where $\bar{u}_{j}(x)$ is the $\bar{u}_{j}^{R i}(x)$ in H7. Since $p^{i}(x)$ attracts $\bar{u}_{0}(x)$, cf. H9, (i), the solution $u_{0}^{i}(x, \tau)$ of $(5.8)$ approaches $p^{i}(x)$ exponentially as $\tau \rightarrow \infty$. From a standard perturbation theory, the linear variational equation

$$
u_{\tau}=f_{0 u}\left(u_{0}, x\right) u
$$

is exponentially stable. We now proceed by induction. Assume that $u_{j}, 0 \leq j \leq$ $k-1$, have been solved with $\left|D_{x}^{\alpha} u_{j}(x, \tau)\right|_{C^{m}([0, \infty))} \leq C_{j \alpha m}$, for all $\alpha \geq 0, m \geq 0$. Rewrite $\left(5.8_{k}\right)$ as

$$
u_{k \tau}=f_{0 u}\left(u_{0}, x\right) u_{k}+h_{k}\left(u_{0}, u_{1}, \ldots, u_{k-1}\right) .
$$

It is easy to see that $\left|D_{x}^{\alpha} h_{k}\right|_{C^{m}([0, \infty))} \leq \infty$ for all $\alpha \geq 0, m \geq 0$. Equation (5.10) with initial condition (5.9) then has a unique solution $u_{k}$ that satisfies

$$
\left|D_{x}^{\alpha} u_{k}(x, \tau)\right|_{C^{m}([0, \infty))} \leq C,
$$

for all $\alpha, m \geq 0$, uniformly with respect to $x$.

For $\alpha=0$, estimate (5.11) comes from the variation of constant formula and the exponential stability of the evolution operator for (5.10). For $\alpha \neq 0$, differentiate (5.10) with respect to $x \alpha$-times and consider the equation for $D_{x}^{\alpha} u_{k}$. (5.11) then follows easily. We have proved the following. 
Theorem 5.3. Assume H1, H7, and H9 (i). Then there exists formal series

$$
u^{R S i}(x, \tau, \epsilon)=\sum_{j=0}^{\infty} \epsilon^{j} u_{j}^{R S i}(x, \tau), \quad u_{j}^{R S i}(x, 0)=\bar{u}_{j}^{R i}(x),
$$

for $\bar{\eta}^{i-1} \leq x \leq \bar{\eta}^{i}, 1 \leq i \leq r$, such that $u^{R S i}$ formally satisfies (5.1). The series can be obtained by recursively solving the system of ODEs (5.8)-(5.8k), $k \geq 1$, with the initial condition (5.9). Furthermore, each $u_{j}^{R S i}$ is $C^{m}$ bounded jointly in $x$ and $\tau$ for all $m \geq 0$.

5.3. Formal series in $(S S)^{i}$ and matching of $(S S)^{i}$ with $(S R)^{i}$. The position of the wave front $\eta^{i}(t, \epsilon)=\sum_{j=0}^{\infty} \epsilon^{i} \eta_{j}^{i}(t)$ depends on its initial condition $\eta^{i}(0, \epsilon)=$ $\sum_{j=0}^{\infty} \epsilon^{j} \bar{\eta}_{j}^{i}$. We will show in this section that $\left\{\bar{\eta}_{j}^{i}\right\}_{j=0}^{\infty}$ is determined by the matching of expansions in $(S S)^{i}$ and $(S R)^{i}$. In the fast time variable $\tau=t / \epsilon$, from a formal expansion,

$$
\stackrel{*}{\eta}^{i}(\tau, \epsilon)=\sum_{j=0}^{\infty} \epsilon^{j} \stackrel{*}{\eta}_{j}^{i}(\tau) \stackrel{\text { def }}{=} \eta^{i}(t, \epsilon)=\eta^{i}(\epsilon \tau, \epsilon)
$$

Each $\stackrel{*}{\eta}_{j}^{i}(\tau)$ is a polynomial of degree $j$. In fact, from $\sum_{j=0}^{\infty} \epsilon^{j} \stackrel{*}{\eta}_{j}^{i}(\tau)=\sum_{j=0}^{\infty} \epsilon^{j} \eta_{j}^{i}(\epsilon \tau)$, we have

$$
\stackrel{*}{\eta}_{j}^{i}(\tau)=\sum_{\ell=0}^{j} \eta_{j-\ell}^{i(\ell)}(0) \tau^{\ell} / \ell !
$$

where $(\ell)$ denotes the $\ell$-th derivative with respect to $t$. In particular,

$$
\stackrel{*}{\eta}_{j}^{i}(0)=\eta_{j}^{i}(0)=\bar{\eta}_{j}^{i}
$$

The stretched variable $\xi=\left(x-\sum_{j=0}^{\infty} \epsilon^{j} \stackrel{*}{\eta}_{j}^{i}(\tau)\right) / \epsilon$ is used to express the solution $u^{S S i}(\xi, \tau, \epsilon)$. When $\tau=0$, in the new variable, the initial data are

$$
\bar{u}^{S i}(\xi, \epsilon)=\sum_{j=0}^{\infty} \epsilon^{j} \bar{u}_{j}^{S i}(\xi) \stackrel{\text { def }}{=} \bar{u}(x, \epsilon)=\bar{u}\left(\epsilon \xi+\sum_{j=0}^{\infty} \epsilon^{j} \bar{\eta}_{j}^{i}, \epsilon\right) .
$$

Let now $\bar{\eta}_{0}^{i}=\bar{\eta}^{i}$. Recall the definition of $\overline{\bar{u}}^{S i}(\xi, \epsilon)=\sum_{j=0}^{\infty} \epsilon^{j} \overline{\bar{u}}^{S i}(\xi)$ It is easy to see that

$$
\begin{gathered}
\bar{u}^{S i}(\xi, \epsilon)=\overline{\bar{u}}^{S i}\left(\xi+\sum_{j=0}^{\infty} \epsilon^{j} \bar{\eta}_{j+1}^{i}, \epsilon\right) . \\
\bar{u}_{0}(\xi)=\overline{\bar{u}}_{0}\left(\xi+\bar{\eta}_{1}^{i}\right), \\
\bar{u}_{1}(\xi)=\overline{\bar{u}}_{1}\left(\xi+\bar{\eta}_{1}^{i}\right)+\overline{\bar{u}}_{0 \xi}\left(\xi+\bar{\eta}_{1}^{i}\right) \bar{\eta}_{2}^{i}, \\
\cdots \\
\bar{u}_{k}(\xi)=\overline{\bar{u}}_{k}\left(\xi+\bar{\eta}_{1}^{i}\right)+\ldots+\overline{\bar{u}}_{0 \xi}\left(\xi+\bar{\eta}_{1}^{i}\right) \bar{\eta}_{k+1}^{i} .
\end{gathered}
$$


Observe that in $\left(5.15_{k}\right)$, the ... comprises of terms containing only $\left\{\bar{\eta}_{j}^{i}\right\}_{j=1}^{k}$.

Let the solution in $(S S)^{i}$ be

$$
u^{S S i}(\xi, \tau, \epsilon)=u^{S S i}\left(\left(x-\stackrel{*}{\eta}^{i}(\tau, \epsilon)\right) / \epsilon, \tau, \epsilon\right)=\sum_{j=0}^{\infty} \epsilon^{j} u_{j}^{S S i}(\xi, \tau)
$$

that satisfies

$$
u_{\tau}=u_{\xi \xi}+\frac{1}{\epsilon} D_{\tau} \stackrel{*}{\eta}^{i}(\tau, \epsilon) \cdot u_{\xi}+f\left(u, \epsilon \xi+\stackrel{*}{\eta}^{i}(\tau, \epsilon), \epsilon\right)
$$

Observe that

$$
\frac{1}{\epsilon} D_{\tau} \eta^{*}(\tau, \epsilon)=\sum_{j=0}^{\infty} \epsilon^{j} \eta_{j+1}^{* i(1)}(\tau)=\sum_{j=0}^{\infty} \epsilon^{j}\left(\sum_{\ell=0}^{j} \eta_{j-\ell}^{i(\ell+1)}(0) \tau^{\ell} / \ell !\right)
$$

where (1) denotes derivatives with respect to $\tau$. Notice that $\stackrel{*}{\eta}_{j+1}^{i(1)}(\tau)$ is a polynomial of degree $j$. Expanding (5.16) and using (5.13) and (5.17), we have a system for $\sum_{j=0}^{\infty} \epsilon^{j} u_{j}^{S S i}$. (Recall $\eta_{0}^{i(1)}(0)=V^{i}\left(\bar{\eta}^{i}\right)$ from $(4.15)$, and $V^{i}\left(\bar{\eta}^{i}\right)=0$ for $i=0, r$.)

$$
\begin{gathered}
u_{0 \tau}=u_{0 \xi \xi}+V^{i}\left(\bar{\eta}^{i}\right) u_{0 \xi}+f_{0}\left(u_{0}, \bar{\eta}^{i}\right), \\
u_{1 \tau}=u_{1 \xi \xi}+V^{i}\left(\bar{\eta}^{i}\right) u_{1 \xi}+f_{0 u}\left(u_{0}, \bar{\eta}^{i}\right) u_{1} \\
+\stackrel{*}{\eta}_{2}^{i(1)}(\tau) u_{0 \xi}+f_{0 x}\left(u_{0}, \bar{\eta}^{i}\right)\left(\xi+\stackrel{*}{\eta}_{1}^{i}(\tau)\right)+f_{1}\left(u_{0}, \bar{\eta}^{i}\right), \\
\cdots \\
u_{k \tau}=u_{k \xi \xi}+V^{i}\left(\bar{\eta}^{i}\right) u_{k \xi}+f_{0 u}\left(u_{0}, \bar{\eta}^{i}\right) u_{k} \\
+\sum_{j=1}^{k} \stackrel{*}{\eta}_{j+1}^{i(1)}(\tau) u_{k-j, \xi}+\sum C_{\alpha \beta \gamma \delta} D_{u}^{|\alpha|} D_{x}^{|\beta|+\gamma} f_{\delta}\left(u_{0}, \bar{\eta}^{i}\right) u^{\alpha} \eta^{\beta} \xi^{\gamma} .
\end{gathered}
$$

Here $\alpha=\left(\alpha_{1}, \ldots, \alpha_{k-1}\right), u^{\alpha}=u_{1}^{\alpha_{1}} \ldots u_{k-1}^{\alpha_{k-1}}, \beta=\left(\beta_{1}, \ldots, \beta_{k}\right), \eta^{\beta}=\stackrel{*}{\eta}_{1}^{\beta} \ldots \stackrel{*}{\eta}_{k}^{\beta_{k}}$, $\gamma, \delta \geq 0$ are integers, $\delta+\gamma+\sum_{j=1}^{k-1}\left(\alpha_{j}+\beta_{j}\right) \cdot j+k \beta_{k}=k, C_{\alpha \beta \gamma \delta}$ is a constant. The initial conditions for $u_{0}, u_{1}, \ldots, u_{k}$ are given in $(5.15)-\left(5.15_{k}\right), k \geq 1$.

The existence of local solutions for $(5.18)-\left(5.18_{k}\right), k \geq 1$, follows from the theory of abstract parabolic equations and analytic semigroup. Let $A u=u_{\xi \xi}+V^{i}\left(\bar{\eta}^{i}\right) u_{\xi}$. Let $1 \leq i \leq r-1$ first. Consider (5.18) in $X=B_{\mathbb{R}}(w)$. Then $A$ is sectorial with $D_{A}=B_{\mathbb{R}}^{2}(w)$. Since $u_{0}(\xi, 0)=\bar{u}_{0}^{i}(\xi) \in D_{A},(5.18)$ admits a unique solution

$$
u \in C^{1}\left(\left[0, t_{0}\right]: X\right) \cap C\left(\left[0, t_{0}\right]: D_{A}\right)
$$

for some $t_{0}>0$. If $i=0$ or $r$, the same conclusion hold but $X=B_{\mathbb{R}^{ \pm}}(w)$ and $D_{A}=B_{\mathbb{R}^{ \pm}}(w) \cap(B C)$. However, due to H9, the solution $u_{0}^{S S i}(\xi, \tau)$ exists for any $t_{0}>0$ and approaches $q^{i}\left(\xi+c, \bar{\eta}^{i}\right)$ as $\tau \rightarrow \infty$, for some constant $c \in \mathbb{R}$. When $1 \leq i \leq r-1,\left(5.18_{k}\right), k \geq 1$, is considered in $X=E_{\mathbb{R}}(w)$ and $A$ is sectorial with $D_{A}=E_{\mathbb{R}}^{2}(w)$. When $i=0, r, X=E_{\mathbb{R}^{ \pm}}(w)$ and $D_{A}=E_{\mathbb{R}^{ \pm}}^{2} \cap(B C)$. Since $u_{k}(\xi, 0)=\bar{u}_{k}^{i}(\xi) \in D_{A}$, and it can be seen by induction that the right hand side of $\left(5.18_{k}\right)$ is in $D_{A}\left(\frac{1}{2}\right),\left(5.18_{k}\right)$ admits a solution $u$ that satisfies (5.19). Since the evolution operator of $\left(5.18_{k}\right)$ is not asymptotically stable, and the nonhomogeneous terms are of $O\left((1+\tau)^{k}\right)$ in $D_{A}\left(\frac{1}{2}\right)$, in general, we can show $\left|u_{k}\right|_{E^{2}\left(1+|\xi|^{k}\right)}=$ 
$O\left((1+\tau)^{k+1}\right)$. However, a better result can be obtained by considering the matching of $(S S)^{i}$ and $(S R)^{i}$. In our case, $u_{k}(\xi, \tau)$ exists for all $\tau \in[0, \infty)$ and satisfies $\left|D_{\tau}^{\alpha} u_{k}(\xi, \tau)\right|+\left|D_{\xi}^{\beta} u_{k}(\xi, \tau)\right| \leq C\left(1+|\xi|^{k}+\tau^{k}\right)$ for $\alpha \leq 1, \beta \leq 2$.

For the purpose of matching we expand $u^{S R i}(\xi, t, \epsilon)=\sum_{j=0}^{\infty} \epsilon^{j} u_{j}^{S R i}(\xi, t)$ in the fast time $\tau=t / \epsilon$.

$$
u^{S R i}(\xi, \epsilon \tau, \epsilon)=u^{*} S R i(\xi, \tau, \epsilon)=\sum_{j=0}^{\infty} \epsilon^{j} \stackrel{*}{u}_{j}^{S R i}(\xi, \tau)
$$

Since $u(x, t, \epsilon)=\stackrel{*}{u}^{S R i}((x-\stackrel{*}{\eta}(\tau, \epsilon)) / \epsilon, \tau, \epsilon), \quad \stackrel{*}{u} S R i(\xi, \tau, \epsilon)$ formally satisfies the same equations as (5.16), therefore $\sum_{j=0}^{\infty} \epsilon^{j} \stackrel{*}{u}_{j}^{S R i}(\xi, \tau)$ formally satisfy system (5.18), $\left(5.18_{1}\right), \ldots,\left(5.18_{k}\right), \ldots$. Also from

$$
\sum_{j=0}^{\infty} \epsilon^{j} u_{j}^{S R i}(\xi, \epsilon \tau)=\sum_{j=0}^{\infty} \epsilon^{j} \stackrel{*}{u}_{j}^{S R i}(\xi, \tau),
$$

and $D_{t}^{\ell} u_{j}^{S R i}(\xi, t) \in E^{m}\left(1+|\xi|^{j}\right)$ for all $\ell, m \geq 0, \stackrel{*}{u}_{j}^{S R i}$ is a polynomial in $\tau$, in the form

$$
\stackrel{*}{u}_{j}^{S R i}=\sum_{\ell=0}^{j} \stackrel{*}{u}_{j \ell}, \text { with }\left|\stackrel{u}{j \ell}_{j \ell}\right|_{E^{m}\left(1+|\xi|^{\ell}\right)} \leq C \tau^{j-\ell} \text {, for all } m \geq 0 .
$$

In particular, ${ }_{j}^{*}{ }_{j}^{S R i} \in O\left(1+|\xi|^{j}+\tau^{j}\right)$.

Observe here that $\left\{u_{j}^{S R i}\right\}_{0}^{\infty}$ has not been determined since $\left\{\bar{\eta}_{j}^{i}\right\}_{1}^{\infty}$ is still unknown. But knowing $\left\{\bar{\eta}_{j}^{i}\right\}_{0}^{k}$ suffices to compute $\left\{u_{j}^{S R i}\right\}_{0}^{k}$ and $\left\{\eta_{j}^{i}(t)\right\}_{0}^{k}$.

We now prove by induction that by successively choosing $\left\{\bar{\eta}_{j}^{i}\right\}_{j=1}^{\infty},\left(\bar{\eta}_{j+1}^{i}\right.$ affects initial condition $\bar{u}_{j}(\xi)$, cf. $\left.\left(5.15_{j}\right)\right)$, system $(5.18)-\left(5.18_{j}\right)$ has a unique solution $\left\{u_{j}^{S S i}\right\}_{j=0}^{\infty}$ such that

$$
u_{j}^{S S i}=\sum_{j=0}^{k} u_{j \ell} \text { with }\left\|u_{j \ell}\right\|_{E^{2}\left(1+|\xi|^{\ell}\right)} \leq C\left(1+\tau^{j-\ell}\right),
$$

and

$$
\left|u_{j \ell}-\stackrel{*}{u}_{j \ell}\right|_{E^{2}\left(1+|\xi|^{\ell}\right)} \leq C\left(1+\tau^{j-\ell}\right) e^{-\gamma \tau} .
$$

First, if $1 \leq i \leq r-1,(5.18)$ has a stable stationary solution $q^{i}\left(\xi, \bar{\eta}^{i}\right)$ that attracts $\overline{\bar{u}}_{0}$ modulo spatial shifts. cf. H9. Thus, there exists a unique $\bar{\eta}_{1}^{i}$ such that with $u_{0}(\xi, 0)=\bar{u}_{0}(\xi)=\overline{\bar{u}}\left(\xi+\bar{\eta}_{1}^{i}\right)$,

$$
\left\|u_{0}^{S S i}(\cdot, \tau)-q^{i}\left(\cdot, \bar{\eta}^{i}\right)\right\|_{B_{\mathbb{R}}^{2}(w)} \leq C e^{-\gamma \tau}, \quad \tau \geq 0,
$$

where $w(\xi)=e^{-\gamma|\xi|}$ and $\gamma>0$. If $i=0, r, q^{i}\left(\xi, \bar{\eta}^{i}\right)$ is stable. We choose $\bar{\eta}_{1}^{i}=0$, (5.23) is still valid. Now (5.23) is even stronger than (5.22), $j=\ell=0$. The extra control of the rate of approaching $u_{0}( \pm \infty, \tau)$ as $\xi \rightarrow \pm \infty$ will be used in $\S 6$. At this point, $\left\{u_{j}^{S R i}\right\}_{j=0}^{1}$ and $\left\{\eta_{j}^{i}\right\}_{j=0}^{1}$ have also been determined.

Assume that $\left\{u_{j}^{S S i}\right\}_{j=0}^{k-1}$ and $\left\{\bar{\eta}_{j}^{i}\right\}_{j=0}^{k}$ have been determined and (5.21), (5.22), $0 \leq j \leq k-1$, are satisfied. Therefore, $\eta_{j}^{i}(t), t \geq 0,0 \leq j \leq k$, and all their derivatives at $t=0$ are determined as well as $\left\{u_{j}^{S R i}\right\}_{0}^{k}$ and $\left\{{ }_{u}^{*}{ }_{j}^{S R i}\right\}_{0}^{k}$. From (5.13) 
and (5.17), $\stackrel{*}{\eta}_{j}^{i}(\tau)$ and $\stackrel{*}{\eta}_{j+1}^{i(1)}(\tau), 0 \leq j \leq k$, are determined, which will be used in $\left(5.18_{k}\right)$. We now rewrite $\left(5.18_{k}\right)$, which is satisfied by $u_{k}^{S S i}$ and $\underset{k}{*} \underset{k}{S R i}$ as the following:

$$
\begin{aligned}
& u_{k \tau}-u_{k \xi \xi}-V^{i}\left(\bar{\eta}^{i}\right) u_{k \xi}-f_{0 u}\left(u_{0}, \bar{\eta}^{i}\right) u_{k}=g_{k}\left(u_{0}, \ldots, u_{k-1}\right), \\
& \stackrel{*}{u}_{k t}-\stackrel{*}{u}_{k \xi \xi}-V^{i}\left(\bar{\eta}^{i}\right) \stackrel{*}{u_{k \xi}}-f_{0 u}\left(\stackrel{*}{u}_{0}, \bar{\eta}^{i}\right) \stackrel{*}{u_{k}}=g_{k}\left(\stackrel{*}{u}_{0}, \ldots, \stackrel{*}{u}_{k-1}\right) .
\end{aligned}
$$

Since $g_{k}\left(u_{0}, \ldots, u_{k-1}\right)$ is a polynomial in $\left(u_{1}, \ldots, u_{k-1}\right)$ and $\left(u_{1 \xi}, \ldots, u_{k-1, \xi}\right)$, using (5.20)-(5.22) we can verify that

$$
g_{k}\left(u_{0}, \ldots, u_{k-1}\right)-g_{k}\left(\stackrel{*}{u}_{0}, \ldots, \stackrel{*}{u}_{k-1}\right)=\sum_{j=0}^{k} g_{k j}
$$

with $\left\|g_{k j}\right\|_{E^{1}\left(1+\|\xi\|^{j}\right)} \leq C\left(1+\tau^{k-j}\right) e^{-\gamma \tau}$. Let $\Delta u=u_{k}-\stackrel{*}{u}_{k}$.

Denote

$$
\begin{aligned}
L^{i} u & =u_{\xi \xi}+V^{i}\left(\eta^{i}\right) u+f_{\text {ou }}\left(u_{0}^{i}, \bar{\eta}^{i}\right) u \\
\Delta u_{\tau}-L^{i} \Delta u & =\sum_{j=0}^{k}\left[f_{0 u}\left(u_{0}, \bar{\eta}^{i}\right)-f_{0 u}\left(u_{0}^{*}, \bar{\eta}^{i}\right)\right] \stackrel{*}{u}_{k j}+\sum_{j=0}^{k} g_{k j} \\
& =\sum_{j=0}^{k} G_{k j} .
\end{aligned}
$$

At this point all the terms in the right hand side of (5.24) are known. Observe that the initial data for $\Delta u$ has the form $u_{k}(\xi, 0)-\stackrel{*}{u}_{k}(\xi, 0)=\varphi(\xi)+\overline{\bar{u}}_{0 \xi}\left(\xi+\bar{\eta}_{1}^{i}\right) \bar{\eta}_{k+1}^{i}$, where $\varphi \in E^{2}\left(1+|\xi|^{k}\right)$. Recall that $\bar{\eta}_{0}^{0}=a, \bar{\eta}_{o}^{r}=b, \bar{\eta}_{j}^{i}=0$ for all $j \geq 1$ if $i=0, r$.

Lemma 5.4. (i) Let $1 \leq i \leq r-1$. Consider

$$
\begin{aligned}
u_{\tau} & =L^{i} u+h, \\
u(\xi, 0) & =\varphi(\xi)+\overline{\bar{u}}_{0 \xi}\left(\xi+\bar{\eta}_{1}^{i}\right) \eta,
\end{aligned}
$$

where $h: \mathbb{R}^{+} \rightarrow E_{\mathbb{R}}^{1}\left(1+|\xi|^{j}\right)$ is continuous with $|h(\tau)|_{E^{1}\left(1+|\xi|^{j}\right)} \leq C\left(1+\tau^{\ell}\right) e^{-\gamma \tau}$ and $\varphi \in E_{\mathbb{R}}^{2}\left(1+|\xi|^{j}\right)$. Then there exists a unique $\eta \in \mathbb{R}$ such that there exists a unique solution $u$ to (5.25) with $|u(\tau)|_{E_{\mathrm{p}}^{2}\left(1+|\xi|^{j}\right)} \leq C\left(1+\tau^{\ell}\right) e^{-\gamma \tau}$.

(ii) Let $i=0$ or $r$. Consider (5.25) with $\bar{\eta}_{1}^{i}=\eta=0$, where $h: \mathbb{R}^{+} \rightarrow$ $E_{\mathbb{R}^{ \pm}}^{1}\left(1+|\xi|^{j}\right)$ is continuous with $|h(\tau)|_{E_{\mathbb{R}^{ \pm}}^{1}\left(1+|\xi|^{j}\right)} \leq C\left(1+\tau^{\ell}\right) e^{-\gamma \tau}$ and $\varphi \in$ $E_{\mathbb{R}^{ \pm}}^{2}\left(1+|\xi|^{j}\right) \cap(B C)$. Then there exists a unique solution $u$ to (5.25) such that $u$ : $\mathbb{R}^{+} \rightarrow E_{\mathbb{R}^{ \pm}}^{2}\left(1+|\xi|^{j}\right) \cap(B C)$ is continuous and $|u(\tau)|_{E_{\mathbb{R}^{ \pm}}^{2}\left(1+|\xi|^{j}\right)} \leq C\left(1+\tau^{\ell}\right) e^{-\gamma \tau}$.

We now write $\Delta u_{k}=\sum_{j=0}^{k} \Delta u_{k j}$ where $\Delta u_{k j}$ satisfies

$$
\begin{aligned}
& u_{\tau}-L^{i} u=\left[f_{0 u}\left(u_{0}^{i}, \bar{\eta}^{i}\right)-f_{0 u}\left(\stackrel{*}{u}_{0}^{i}, \bar{\eta}^{i}\right)\right] \stackrel{*}{u}_{k j}+g_{k j}=G_{k j} . \\
& u(\xi, 0)=\left\{\begin{aligned}
\overline{\bar{u}}_{0 \xi}\left(\xi+\bar{\eta}_{1}^{i}\right) C_{j}, & \text { if } 0 \leq j \leq k-1, \\
\varphi(\xi)+\overline{\bar{u}}_{0 \xi}\left(\xi+\bar{\eta}_{1}^{i}\right) C_{k}, & \text { if } j=k .
\end{aligned}\right.
\end{aligned}
$$


Let $1 \leq i \leq r-1$ first. Since $\varphi \in E^{2}\left(1+|\xi|^{k}\right)$ and $\left|G_{k j}\right|_{E_{\mathbb{R}}^{1}\left(1+|\xi|^{j}\right)} \leq$ $C\left(1+\tau^{k-j}\right) e^{-\gamma \tau}$, from Lemma 5.4, (i), there exists a unique $C_{j}, 0 \leq j \leq k$, such that the unique solution $\Delta u_{k j}$ of (5.26), (5.27) satisfies

$$
\left|\Delta u_{k j}\right|_{E_{\mathbb{R}}^{2}\left(1+|\xi|^{j}\right)} \leq C\left(1+\tau^{k-j}\right) e^{-\gamma \tau} .
$$

Let $i=0$ or $r$ next. Then $\left.\varphi(\xi)=\overline{\bar{u}}_{k}(\xi)=\bar{u}_{k}(\xi) \in E_{\mathbb{R}^{ \pm}}^{2}(1+|\xi|)^{k}\right) \cap(B C)$ and $\left|G_{k j}\right|_{E_{\mathbb{R}^{ \pm}}^{1}\left(1+|\xi|^{j}\right)} \leq C\left(1+\tau^{k-j}\right) e^{-\gamma \tau}$ and $C_{j}=0,0 \leq j \leq k$. From Lemma 5.4, (ii), the unique solution $\Delta u_{k j}$ of (5.26), (5.27) satisfies the Neumann boundary condition at $\xi=0$ and

$$
\left|\Delta u_{k j}\right|_{E_{\mathbb{R}^{ \pm}}^{2}\left(1+|\xi|^{j}\right)} \leq C\left(1+\tau^{k-j}\right) e^{-\gamma \tau} .
$$

In all the cases let $\bar{\eta}_{j+1}^{i}=\sum_{0}^{k} c_{j}$ and $u_{k j}^{i}=\Delta u_{k j}+\stackrel{*}{u}_{k j}$. From (5.20), we have (5.21) for $j=k$. Thus, (5.21) and (5.22) have been proved by the induction.

We summarize the results in

Theorem 5.5. Assume $\mathrm{H} 1-\mathrm{H} 5$. For each $0 \leq i \leq r$ assume the initial data $\overline{\bar{u}}^{S i}(\xi, \epsilon)=\sum \epsilon^{j} \overline{\bar{u}}_{j}^{S i}(\xi)$ satisfies $\mathrm{H} 7$ and $\mathrm{H} 9$, (ii). Then there exist formal series

$$
\begin{gathered}
u^{S S i}(\xi, \tau, \epsilon)=\sum \epsilon^{j} u_{j}^{S S i}(\xi, \tau), \quad u_{j}^{S S i}(\xi, 0)=\bar{u}_{j}^{S i}(\xi), \\
\sum \epsilon^{j} \bar{\eta}_{j}^{i}, \quad \bar{\eta}_{0}^{i}=\bar{\eta}^{i} .
\end{gathered}
$$

Here $\bar{\eta}^{i}$ is determined by the layer position of the initial data. (5.30) formally satisfies (5.16) where ${ }^{*}{ }^{i}(\tau, \epsilon)=\eta^{i}(\epsilon \tau, \epsilon)$ is the expansion of the layer position in the variable $\tau=t / \epsilon$, see (5.12). Furthermore (5.30) is recursively determined by (5.18)-(5.18k), $k \geq 1$, with the initial conditions (5.15)-(5.15k), $k \geq 1$, in the space $u_{j}^{S S i} \in E^{2}\left(1+|\xi|^{j}\right)$, where $\bar{\eta}_{j}^{i}, j \geq 1$, is chosen such that for any integers $\alpha \leq 2, \beta \leq 1$,

$$
\begin{aligned}
\left|\partial_{\xi}^{\alpha} u_{j}^{S S i}\right|+\left|\partial_{\tau}^{\beta} u_{j}^{S S i}\right| & \leq C\left(1+|\xi|^{j}+\tau^{j}\right), \\
\left|u_{j}^{S S i}-{ }_{u}^{*}{ }_{j}^{S R i}\right| & \leq C\left(\left(1+|\xi|^{j}+\tau^{j}\right) e^{-\gamma \tau},\right.
\end{aligned}
$$

for some $\gamma>0$.

\section{Matching of LaYer SOlutions, CONSTRUCtion OF PSEUdo SOlutions}

6.1. The matching of $(R S)^{i}$ and $(R R)^{i}$.

Theorem 6.1. There exists $\gamma>0$ such that for any integers $\alpha, \beta, j \geq 0$,

$$
\left|\partial_{\tau}^{\alpha} \partial_{x}^{\beta}\left(u_{j}^{R S i}(x, \tau)-u_{j}^{R R i}(x)\right)\right| \leq C_{\alpha \beta j} e^{-\gamma \tau},
$$

uniformly for all $x \in\left[\bar{\eta}^{i-1}, \bar{\eta}^{i}\right], 1 \leq i \leq r$.

When $j=0$, Theorem 6.1 is a consequence of the exponential stability of $p^{i}(x), 1 \leq i \leq r$, as a stationary solution to (5.8). By induction, we can prove that the nonhomogeneous term and the coefficient $f_{0 u}\left(u_{0}, x\right)$ in $\left(5.8_{k}\right)$ approach the corresponding terms in $\left(4.10_{k}\right)$ exponentially as $\tau \rightarrow \infty$. The desired result then follows easily. Details are omitted. 
6.2. The matching of $(S S)^{i}$ and $(S R)^{i}$. The matching of expansions in $(S S)^{i}$ and $(S R)^{i}$ has been obtained in $\S 5.3$. For convenience, we state the result in the following

Theorem 6.2. Let $\Delta u_{j}^{i}=u_{j}^{S S i}-\stackrel{*}{u}{ }_{j}^{S R i}$, where $\sum_{0}^{\infty} \epsilon^{j}{ }_{u}^{*}{ }_{j}^{S R i}$ is the expansion of $\sum_{0}^{\infty} \epsilon^{j} u_{j}^{S R i}(\xi, t)$ in the variable $\tau=t / \epsilon$. Then there exists $\gamma>0$ such that for any nonnegative integers $\alpha \leq 2, \beta \leq 1$,

$$
\left\{\left|\partial_{\xi}^{\alpha} \Delta u_{j}^{i}\right|+\left|\partial_{\tau}^{\beta} \Delta u_{j}^{i}\right|\right\} \leq C_{j}\left(1+|\xi|^{j}+\tau^{j}\right) e^{-\gamma \tau} .
$$

6.3. The matching of $(S R)^{i}$ with $(R R)^{i}$ and $(R R)^{i+1}$. Let the inner expansion of the outer formal solutions in $(R R)^{i}$ be

$$
\begin{aligned}
& \sum_{j=0}^{\infty} \epsilon^{j} \tilde{u}_{j, 1}^{R R i}(\xi, t)=\sum_{j=0}^{\infty} \epsilon^{j} u_{j}^{R R i}\left(\epsilon \xi+\sum_{\ell=0}^{\infty} \epsilon^{\ell} \eta_{\ell}^{i}(t)\right), \quad 1 \leq i \leq r, \\
& \sum_{j=0}^{\infty} \epsilon^{i} \tilde{u}_{j, 2}^{R R i}(\xi, t)=\sum_{j=0}^{\infty} \epsilon^{j} u_{j}^{R R(i+1)}\left(\epsilon \xi+\sum_{\ell=0}^{\infty} \epsilon^{\ell} \eta_{\ell}^{i}(t)\right), \quad 0 \leq i \leq r-1 .
\end{aligned}
$$

Recall that $\eta_{0}^{0}(t)=a, \eta_{0}^{r}(t)=b$ and $\eta_{\ell}^{0}(t)=\eta_{\ell}^{r}(t)=0$ for all $\ell \geq 1$. From (6.1), we find that $\tilde{u}_{j, \nu}^{R R i}, \nu=1,2$, is a polynomial in $\xi$ of degree $j$, with its coefficients depending smoothly on $\eta_{\ell}^{i}(t), \ell \leq j$. Thus,

$$
\tilde{u}_{k, \nu}^{R R i} \in E_{\mathbb{R}}^{m}\left(1+|\xi|^{k}\right), \quad \nu=1,2,
$$

with the norm bounded uniformly with respect to $t \geq 0$.

Concerning the matching of $(S R)^{i}$ and $(R R)^{i}$, we want to show that there exists $\gamma>0$ such that the following estimates hold.

$$
\begin{aligned}
\left|\partial_{t}^{\alpha}\left(u_{j}^{S R i}(., t)-\tilde{u}_{j, 1}^{R R i}(., t)\right)\right|_{E_{\mathbb{R}^{-}}\left(\left(1+\left.|\xi|\right|^{j}\right) e^{-\gamma|\xi|}\right)} \leq C_{\alpha m j}, & 1 \leq i \leq r, \\
\left|\partial_{t}^{\alpha}\left(u_{j}^{S R i}(., t)-\tilde{u}_{j, 2}^{R R i}(., t)\right)\right|_{E_{\mathbb{R}^{+}}^{m}\left(\left(1+\xi^{j}\right) e^{-\gamma \xi}\right)} \leq C_{\alpha m j}, & 0 \leq i \leq r-1,
\end{aligned}
$$

for all $\alpha, m, j \geq 0$ uniformly uniformly with respect to $t \geq 0$ in the weighted norms. Only the proof of the second estimate, $0 \leq i \leq r-1$, will be presented since the proof of the first is similar.

Notice that $\sum_{j=0}^{\infty} \epsilon^{j} \tilde{u}_{j, 2}^{R R i}(\xi, t)$ formally satisfies

$$
\epsilon \tilde{u}_{t}=\tilde{u}_{\xi \xi}+D_{t} \eta(t, \epsilon) \tilde{u}_{\xi}+f(\tilde{u}, \epsilon \xi+\eta(t, \epsilon), \epsilon) .
$$

This has the same form as (4.12). Expanding in power series of $\epsilon$, the equations for $\left\{\tilde{u}_{j, 2}^{R R i}\right\}_{j=0}^{\infty}$ are precisely $(4.13),\left(4.13_{1}\right), \ldots,\left(4.13_{k}\right), \ldots$. When $j=0$, the equation is

$$
0=V^{i}(x) \tilde{u}_{0 \xi}+\tilde{u}_{0 \xi \xi}+f_{0}\left(\tilde{u}_{0}, x\right) .
$$

The above has a solution $\tilde{u}_{0}(\xi, t)=p^{i}\left(\eta_{0}(t)\right)$ (which is in fact independent of $\xi$ ). We can prove by induction that $\tilde{u}_{j}(\xi, t)=\tilde{U}_{j}\left(\xi, x, x_{1}, \ldots, x_{j}\right)$ where $x=\eta_{0}^{i}(t), \ldots, x_{j}=$ $\eta_{j}^{i}(t)$. Also $D_{y}^{\nu} \tilde{U}_{j} \in E^{m}\left(1+|\xi|^{j}\right)$ where $y=\left(x, \ldots, x_{j}\right)$ and $\nu=\left(\nu_{0}, \nu_{1}, \ldots, \nu_{j}\right)$. These properties are similar to those of $U_{j}$, see $\S 5$. 
The assertion is clearly valid if $j=0$. Suppose it is valid for $0 \leq j \leq k-1$. Then $\tilde{u}_{k}$ satisfies the following equation, similar to (4.16),

$$
\tilde{u}_{k \xi \xi}+V^{i}(x) \tilde{u}_{k \xi}+f_{0 u}\left(\tilde{u}_{0}, x\right) \tilde{u}_{k}+V_{k} \tilde{u}_{0 \xi}=\tilde{h}_{k}\left(\xi, \tilde{U}_{0}, \ldots \tilde{U}_{k-1}, x_{1}, \ldots, x_{k}\right),
$$

where $\tilde{h}$ has the same form as $h_{k}$ in (4.16) except $U_{j}$ is replaced by $\tilde{U}_{j}$. Notice that the homogeneous equation associated to (6.4) has an exponential dichotomy in $\mathbb{R}$, and the nonhomogeneous terms are in $E^{m}\left(1+|\xi|^{k}\right)$. Thus (6.4) has a unique solution $\tilde{U}_{k}\left(., x, \ldots, x_{k}\right) \in E^{m}\left(1+|\xi|^{k}\right)$ that depends smoothly on $\left(x, \ldots, x_{k}\right)$, see Lemma 2.3, (i). Since $\tilde{u}_{k} \in E^{m}\left(1+|\xi|^{k}\right)$ is a solution to (6.4), thus $\tilde{u}_{k}=\tilde{U}_{k}\left(., x, \ldots, x_{k}\right)$. The proof is complete.

For $0 \leq i \leq r-1$, let $\Delta u_{k} \stackrel{\text { def }}{=} u_{k}-\tilde{u}_{k}$. We claim that $\Delta u_{j}=\Delta U_{j} \stackrel{\text { def }}{=}$ $U_{j}\left(\xi, x, \ldots, x_{j}\right)-\tilde{U}_{j}\left(\xi, x, \ldots, x_{j}\right)$ satisfies

$$
D_{y}^{\nu} \Delta U_{j}=O\left(\left(1+\xi^{j}\right) e^{-\gamma \xi}\right)
$$

where $\gamma>0$ is a constant, $D_{y}^{\nu}=D_{x}^{\nu_{0}} D_{x_{1}}^{\nu_{1}} \cdots D_{y_{j}}^{\nu_{j}}$. For $k=0$ and $\nu=0, \Delta u_{0}=$ $q^{i}(\xi, x)-p^{i+1}(x)=U_{0}(\xi, x)-\tilde{U}_{0}(\xi, x)$. Obviously we have $\Delta u_{0}=O\left(e^{-\gamma \xi}\right)$. When $k=0$ and $\nu=1, Z \stackrel{\text { def }}{=} D_{x} \Delta U_{0}$ satisfies

$$
\begin{aligned}
0 & =V^{i}(x) Z_{\xi}+Z_{\xi \xi}+f_{0 u}\left(p^{i+1}(x), x\right) Z+f_{0 x}\left(q^{i}(\xi, x), x\right)-f_{0 x}\left(p^{i}(x), x\right) \\
& +\left[f_{0 u}\left(q^{i}(\xi, x), x\right)-f_{0 u}\left(p^{i}(x), x\right)\right] \partial_{x} q^{i}(\xi, x)+\partial_{x} V^{i}(x) \Delta U_{0} .
\end{aligned}
$$

The associated homogeneous equation for $Z$ has an exponential dichotomy in $\mathbb{R}$ and the nonhomogeneous terms are of $O\left(e^{-\gamma \xi}\right)$. If $\gamma$ is smaller than the exponential coefficient of the dichotomy, then $Z=O\left(e^{-\gamma \xi}\right)$, see Lemma 2.3, (i). Assuming now $k=0$ and $\nu>1$, we can write a similar equation for $Z \stackrel{\text { def }}{=} D_{x}^{\nu} \Delta U_{0}$ and prove (6.5) by induction on $\nu$.

Suppose now (6.5) has been proved for $0 \leq j \leq k-1, k \geq 1$. Then $\Delta u_{k}$ satisfies (6.6)

$$
\begin{aligned}
Z_{\xi \xi}+ & V^{i}(x) Z_{\xi}+f_{0 u}\left(\tilde{u}_{0}, x\right) Z \\
= & h_{k}\left(\xi, U_{0}, \ldots, U_{k-1}, x, \ldots, x_{k}\right)-\tilde{h}_{k}\left(\xi, \tilde{U}_{0}, \ldots, \tilde{U}_{k-1}, x, \ldots, x_{k}\right) \\
& +\left[f_{0 u}\left(\tilde{u}_{0}, x\right)-f_{0 u}\left(q^{i}(\xi, x), x\right)\right] u_{k}+V_{k}\left[\tilde{u}_{0 \xi}-q_{\xi}^{i}(\xi, x)\right] .
\end{aligned}
$$

For $1 \leq i \leq r-1,(6.6)$ is considered for $\xi \in \mathbb{R}$. The right hand side and its derivatives with respect to $y=\left(x, x_{1}, \ldots, x_{k}\right)$ are in $E^{m}\left(1+|\xi|^{k}\right)$ and

$E^{m}\left(\left(1+|\xi|^{k}\right) e^{-\gamma \xi}\right)$, due to the induction assumption. Recall that $\tilde{u}_{0}=p^{i+1}$ and $L_{p^{i+1}}$ has an exponential dichotomy on $\mathbb{R}$. From Lemma 2.3 (i), (6.6) has a unique solution $Z$ that is in both $E^{m+2}\left(1+|\xi|^{k}\right)$ and $E^{m+2}\left(\left(1+|\xi|^{k}\right) e^{-\gamma \xi}\right)$ and is differentiable with respect to $y$. However, it is known that $U_{k}, \tilde{U}_{k}$, therefore $\Delta U_{k} \in E^{m+2}\left(1+|\xi|^{k}\right)$. We then have $D_{y}^{\nu} \Delta U_{k}=D_{y}^{\nu} Z \in E^{m+2}\left(\left(1+|\xi|^{k}\right) e^{-\gamma \xi}\right)$. When $1 \leq i \leq k-1,(6.5)$ has been proved by induction.

For $i=0, \Delta u_{k}$ satisfies (6.6) for $\xi \in \mathbb{R}^{+}$and the boundary condition

$$
Z_{\xi}(0, y)=-\tilde{U}_{k \xi}(0, y)
$$

where $y=\left(x, x_{1}, \ldots, x_{k}\right)$. From Lemma 2.3 (ii), (6.6) and (6.7) have a unique solution $Z$ that is in $E_{\mathbb{R}^{+}}^{m+2}\left(1+|\xi|^{k}\right)$ and $E_{\mathbb{R}^{+}}^{m+2}\left(\left(1+|\xi|^{k}\right) e^{-\gamma \xi}\right)$ together with all its derivatives. However, we know that $\Delta U_{k}$ is a solution of (6.6)and (6.7) and is in $E^{m+2}\left(1+|\xi|^{k}\right)$. Thus $\Delta U_{k}=Z$. By induction, (6.5) has been proved for $i=0$. 
Since equation (6.6) and (6.7) depend continuously on $y$ that is in a compact subset of $\mathbb{R}^{k+1}$, the norms of $\Delta U$ and all its derivatives in the function spaces are uniformly bounded with respect to $y$. When $\alpha=0$, the second estimate of (6.2) has already been proved. Recall that $\Delta u_{k}(\xi, t)=\Delta U_{k}(\xi, y)$. Since the derivatives of $\eta_{j}^{i}(t)$ are bounded uniformly with respect to $t \geq 0$, when $\alpha \neq 0$, the desired assertion follows from (6.5) and the chain rule of differentiation.

Theorem 6.3. Let $\Delta u_{j}^{i}=u_{j}^{S R i}-\tilde{u}_{j, 1}^{R R i}, 1 \leq i \leq r$, or $u_{j}^{S R i}-\tilde{u}_{j, 2}^{R R i}, 0 \leq i \leq r-1$, where $\tilde{u}_{j, 1}^{R R i}$ and $\tilde{u}_{j, 2}^{R R i}$ are defined in (6.1). Then there exists $\gamma>0$ such that for all integers $\alpha, \beta, j \geq 0$,

$$
\left|\partial_{t}^{\alpha} \partial_{\xi}^{\beta} \Delta u_{j}^{i}\right| \leq C_{\alpha \beta j}\left(1+|\xi|^{j}\right) e^{-\gamma|\xi|}
$$

uniformly with respect to $t \geq 0$.

6.4. Matching of $(S S)^{i}$ with $(R S)^{i}$ and $(R S)^{i+1}$. We use the inner variable $\xi=(x-\stackrel{*}{\eta}(\tau, \epsilon)) / \epsilon$ to expand the outer solutions

$$
\begin{aligned}
\sum_{j=0}^{\infty} \epsilon^{j} u_{j}^{R S i}\left(\epsilon \xi+\stackrel{*}{\eta}^{i}(\tau, \epsilon), \tau\right)=\sum_{j=0}^{\infty} \epsilon^{j} \tilde{u}_{j, 1}^{R S i}(\xi, \tau), & 1 \leq i \leq r, \\
\sum_{j=0}^{\infty} \epsilon^{j} u_{j}^{R S, i+1}\left(\epsilon \xi+\stackrel{*}{\eta}^{i}(\tau, \epsilon), \tau\right)=\sum_{j=0}^{\infty} \epsilon^{j} \tilde{u}_{j, 2}^{R S i}(\xi, \tau), & 0 \leq i \leq r-1 .
\end{aligned}
$$

Both expansions formally satisfy (5.16) as does $u^{S S i}(\xi, \tau, \epsilon)$. Therefore, both satisfy $(5.18)-\left(5.18_{k}\right), k \geq 1$, just as $\sum_{j=0}^{\infty} \epsilon^{j} u_{j}^{S S i}$. It suffices to show the matching of expansions in $(S S)^{i}$ and $(R S)^{i+1}, 0 \leq i \leq r-1$. Let the indices be dropped so that $\tilde{u}_{j}$ denotes $\tilde{u}_{j, 2}^{R S i}$. Then

$$
\begin{gathered}
\tilde{u}_{0 \tau}=f_{0}\left(\tilde{u}_{0}, \bar{\eta}^{i}\right), \quad \tilde{u}(0)=\bar{u}_{0}^{R, i+1}\left(\bar{\eta}^{i}\right), \\
u_{0 \tau}^{S S i}=u_{0 \xi \xi}^{S S i}+V^{i}\left(\bar{\eta}^{i}\right) u_{0 \xi}^{S S i}+f_{0}\left(u_{0}^{S S i}, \bar{\eta}^{i}\right) .
\end{gathered}
$$

Let $\xi \rightarrow+\infty$ in (6.10). Since $u_{0} \in B_{R^{+}}^{2}\left(e^{-\gamma \xi}\right), u_{0 \xi}$ and $u_{0 \xi \xi} \rightarrow 0$ as $\xi \rightarrow \infty$. We have $u_{0 \tau}(+\infty, \tau)=f_{0}\left(u_{0}(+\infty, \tau), \bar{\eta}^{i}\right)$ with $u_{0}(\infty, 0)=\overline{\bar{u}}_{0}(\infty)=\bar{u}_{0}^{R, i+1}\left(\bar{\eta}^{i}\right)$. This is the same as (6.9). Therefore

$$
u_{0}^{S S i}(\infty, \tau)=\tilde{u}_{0}\left(\bar{\eta}^{i}, \tau\right), \quad \tau \geq 0 .
$$

Since $u_{0}^{S S i}: \mathbb{R}^{+} \rightarrow B_{\mathbb{R}}^{2}\left(e^{-\gamma \xi}\right)$ is bounded, we have

$$
\left|u_{0}^{S S i}(\cdot, \tau)-\tilde{u}_{0}(\cdot, \tau)\right|_{E_{\mathbb{R}^{+}}^{2}\left(e^{-\gamma \xi}\right)} \leq C
$$

uniformly with respect to $\tau \geq 0$.

Let $\Delta u_{j}=u_{j}^{S S i}-\tilde{u}_{j, 2}^{R S i}$. We now show by induction that

$$
\Delta u_{j}=\sum_{i=0}^{j} \Delta u_{j \ell}, \text { with }\left|\Delta u_{j \ell}\right|_{E_{\mathbb{R}^{+}}^{2}}\left(\left(1+\xi^{\ell}\right) e^{-\gamma \xi}\right) \leq C\left(1+\tau^{j-\ell}\right) .
$$


Suppose (6.12) has been proved for $0 \leq j \leq k-1$. For $1 \leq i \leq r-1, \Delta u_{k}$ satisfies

$$
\begin{aligned}
\Delta u_{k \tau} & =\Delta u_{k \xi \xi}+V^{i}\left(\bar{\eta}^{i}\right) \Delta u_{k \xi}+f_{0 u}\left(\tilde{u}_{0}, \bar{\eta}^{i}\right) \Delta u_{k} \\
& +\left[f_{0 u}\left(u_{0}^{S S i}, \bar{\eta}^{i}\right)-f_{0 u}\left(\tilde{u}_{0}, \bar{\eta}^{i}\right)\right] u_{k}^{S S i} \\
& +g_{k}\left(u_{0}, \ldots, u_{k-1}\right)-g_{k}\left(\tilde{u}_{0}, \ldots, \tilde{u}_{k-1}\right) .
\end{aligned}
$$

For $i=0, \Delta u_{k}$ satisfies the equations

$$
\begin{aligned}
\Delta u_{k \tau} & =\Delta u_{k \xi \xi}+V^{i}\left(\bar{\eta}^{i}\right) \Delta u_{k \xi}+f_{0 u}\left(u_{0}^{S S i}, \bar{\eta}^{i}\right) \Delta u_{k} \\
& +\left[f_{0 u}\left(u_{0}^{S S i}, \bar{\eta}^{i}\right)-f_{0 u}\left(\tilde{u}_{0}, \bar{\eta}^{i}\right)\right] \tilde{u}_{k} \\
& +g_{k}\left(u_{0}, \ldots, u_{k-1}\right)-g_{k}\left(\tilde{u}_{0}, \ldots, \tilde{u}_{k-1}\right),
\end{aligned}
$$

where $V^{i}=0, \bar{\eta}^{i}=a$. Observe that $g_{k}$ is a polynomial on $u_{1}, \ldots, u_{k-1}, u_{1 \xi}, \ldots$, $u_{k-1, \xi}$ and $\stackrel{*}{\eta}_{1}^{i}, \ldots, \stackrel{*}{\eta}_{k}^{i}$. By the induction assumption, the nonhomogeneous term of (6.13) has the form

$$
G_{k}=\sum_{j=0}^{k} G_{k j}, \quad \text { with }\left|G_{k j}\right|_{E_{\mathbb{R}}^{1}\left(\left(1+\xi^{j}\right) e^{-\gamma \xi)}\right.} \leq C\left(1+\tau^{k-j}\right),
$$

if $1 \leq i \leq r-1$. The nonhomogeneous term in (6.14) has the same form as in (6.15), but the norm has to be replaced by the norm in $E_{\mathbb{R}^{+}}^{1}\left(\left(1+\xi^{j}\right) e^{-\gamma \xi}\right)$.

We need similar decompositions of $\left\{\tilde{u}_{j}\right\}_{j=0}^{\infty}$ and $\left\{\Delta u_{j}(\xi, 0)\right\}_{j=0}^{\infty}$. The expansion of $\sum \epsilon^{j} u_{j}^{R S, i+1}$ into $\sum \epsilon^{j} \tilde{u}_{j}$ can be divided into two steps. Let

$$
\begin{aligned}
& \sum_{j=0}^{\infty} \epsilon^{j} \tilde{\tilde{u}}_{j}(\xi, \tau)=\sum_{j=0}^{\infty} \epsilon^{j} u_{j}^{R S, i+1}\left(\epsilon \xi+\stackrel{\eta}{\eta}_{0}^{i}, \tau\right), \\
& \sum_{j=0}^{\infty} \epsilon^{j} \tilde{u}_{j}(\xi, \tau)=\sum_{j=0}^{\infty} \epsilon^{j} \tilde{\tilde{u}}_{j}\left(\xi+\sum_{\ell=0}^{\infty} \epsilon^{\ell} \stackrel{\eta}{\eta}_{\ell+1}^{i}(\tau), \tau\right) .
\end{aligned}
$$

We then have

$$
\tilde{u}_{k}(\xi, \tau)=\sum_{\delta, \alpha} C_{\alpha \delta} D_{x}^{|\alpha|} \tilde{\tilde{u}}_{\delta}\left(\xi+\eta_{1}^{*}(\tau)\right)\left[\eta^{*}\right]^{\alpha} \stackrel{\text { deff }}{=} \sum_{\delta=0}^{k} \tilde{u}_{k \delta}
$$

Here $\delta \geq 0$ is an integer, $\left[\stackrel{*}{\eta}^{\alpha}\right]^{\alpha}=\left[\stackrel{*}{\eta}_{2}^{i}(\tau)\right]^{\alpha_{1}} \ldots\left[\stackrel{*}{\eta}_{k+1}^{i}(\tau)\right]^{\alpha_{k}}, C_{\alpha \delta}$ is a constant and $\delta+\sum_{j=1}^{k} j \alpha_{j}=k . \tilde{u}_{k \delta}$ consists of derivatives of $\tilde{\tilde{u}}_{\delta}$ only and

$$
\left|\tilde{u}_{k \delta}\right|_{E^{m}\left(\left(1+|\xi|^{\delta}\right)\right)} \leq C_{m k \delta}\left(1+\tau^{k-\delta}\right), \quad m \geq 0 .
$$

In comparison, $\sum \epsilon^{j} \bar{u}_{j}(\xi)$ is also defined in a similar way

$$
\sum_{j=0}^{\infty} \epsilon^{j} \bar{u}_{j}(\xi)=\sum_{j=0}^{\infty} \epsilon^{j} \overline{\bar{u}}_{j}\left(\xi+\sum_{\ell=0}^{\infty} \epsilon^{\ell} \bar{\eta}_{\ell+1}^{i}\right) .
$$


Exactly like (6.16), we have

$$
\begin{aligned}
\bar{u}_{k}(\xi)= & \sum_{\delta, \alpha} C_{\alpha \delta} D_{x}^{|\alpha|} \overline{\bar{u}}_{\delta}\left(\xi+\bar{\eta}_{1}^{i}\right)[\bar{\eta}]^{\alpha} \stackrel{\text { def }}{=} \sum_{\delta=0}^{k} \bar{u}_{k \delta}, \\
& \left|\bar{u}_{k \delta}\right|_{E^{m}\left(1+|\xi|^{\delta}\right)} \leq \bar{C}_{m k \delta} .
\end{aligned}
$$

Recall that $\stackrel{*}{\eta}_{j}^{i}(0)=\bar{\eta}_{j}^{i}$ and $\left|\overline{\bar{u}}_{\delta}(\xi)-\tilde{\tilde{u}}_{\delta}(\xi, 0)\right|_{E^{m}\left(\left(1+|\xi|^{\delta}\right) e^{-\gamma \xi}\right)} \leq C$, cf. H8. We have

$$
\Delta u_{k}(\xi, 0)=\sum_{j=0}^{k} \Delta u_{k j}(\xi, 0) \stackrel{\text { def }}{=} \sum_{j=0}^{k}\left[\bar{u}_{k j}(\xi)-\tilde{u}_{k j}(\xi, 0)\right],
$$

with

$$
\left|\Delta u_{k j}(\cdot, 0)\right|_{E^{m}\left(\left(1+|\xi|^{j}\right) e^{-\gamma \xi}\right)} \leq C .
$$

For $1 \leq i \leq r-1$ we solve

$$
\begin{aligned}
& \Delta u_{k j \tau}=\Delta u_{k j \xi \xi}+V^{i}\left(\bar{\eta}^{i}\right) \Delta u_{k j \xi}+f_{0 u}\left(\tilde{u}_{0}, \bar{\eta}^{i}\right) \Delta u_{k j}+G_{k j}, \\
& \Delta u_{k j}(\xi, 0)=\bar{u}_{k j}(\xi)-\tilde{u}_{k j}(\xi, 0) .
\end{aligned}
$$

For $i=0$, we solve

$$
\begin{gathered}
\Delta u_{k j \tau}=\Delta u_{k j \xi \xi}+V^{i}\left(\bar{\eta}^{i}\right) \Delta u_{k j \xi}+f_{0 u}\left(u_{0}^{S S i}, \bar{\eta}^{i}\right) \Delta u_{k j}+G_{k j} \\
\Delta u_{k j}(\xi, 0)=\bar{u}_{k j}(\xi)-\tilde{u}_{k j}(\xi, 0) \\
\Delta u_{k j \xi}(0, \tau)=-\tilde{u}_{k j \xi}(0, \tau) .
\end{gathered}
$$

Let $1 \leq i \leq r-1$ first. Since $\tilde{u}_{0} \rightarrow p^{i+1}\left(\bar{\eta}^{i}\right)$ as $\tau \rightarrow \infty$, equation (6.21) is exponentially stable in the space $E_{\mathbb{R}}^{2}\left(\left(1+|\xi|^{j}\right) e^{-\gamma \xi}\right)$. Therefore, from Lemma 2.5, with $\theta=1, \beta=\frac{1}{2}$,

$$
\left|\Delta u_{k j}\right|_{E_{\mathbb{R}}^{2}\left(\left(1+|\xi|^{j}\right) e^{-\gamma \xi}\right)} \leq C\left(1+\tau^{k-j}\right) .
$$

Thus (6.12) has been proved for $1 \leq i \leq r-1$.

Let $i=0$. We consider (6.22) in $\xi \geq 0$. The estimate in (6.15) is replaced by

$$
\left|G_{k j}\right|_{E_{\mathbb{R}^{+}}^{1}\left(\left(1+\xi^{j}\right) e^{-\gamma \xi}\right)} \leq C\left(1+\tau^{k-j}\right) .
$$

To solve (6.22) and $\left(6.22_{1}\right)$, let $\tilde{u}_{k j \xi}(0, \tau)=\varphi_{j}(\tau)$. We have $\left|D^{\alpha} \varphi_{j}(\tau)\right| \leq$ $C\left(1+\tau^{k-j}\right), \alpha=0,1$. Let $\Phi(\xi, \tau)$ be the solution to the elliptic system with a boundary condition at $\xi=0$,

$$
\begin{aligned}
& \Phi_{\xi \xi}+V^{i}\left(\bar{\eta}^{i}\right) \Phi_{\xi}+f_{0 u}\left(q^{i}\left(\xi, \bar{\eta}^{i}\right), \bar{\eta}^{i}\right) \Phi=0, \\
& \Phi_{\xi}(0, \tau)=-\varphi_{j}(\tau) .
\end{aligned}
$$

Then from Lemma 2.3(ii), there exists a unique solution $\Phi$ such that

$$
\left|\partial_{\tau}^{\alpha} \Phi\right|_{E_{\mathbb{R}^{+}}^{2}\left(\left(1+\xi^{j}\right) e^{-\gamma \xi}\right)} \leq C\left(1+\tau^{k-j}\right), \quad \alpha=0,1 .
$$

The solution $\Delta u_{k j}=\Phi+\Psi$, with

$$
\begin{aligned}
\Psi_{\tau} & =\Psi_{\xi \xi}+V^{i}\left(\bar{\eta}^{i}\right) \Psi_{\xi}+f_{0 u}\left(u_{0}^{S S i}, \bar{\eta}^{i}\right) \Psi+G_{k j}-\Phi_{\tau} \\
& +\left[f_{0 u}\left(u_{0}^{S S i}, \bar{\eta}^{i}\right)-f_{0 u}\left(q^{i}\left(\xi, \bar{\eta}^{i}\right), \bar{\eta}^{i}\right)\right] \Phi, \\
\Psi_{\xi}(0, \tau) & =0 \\
\Psi(\xi, 0) & =\Delta u_{k j}(\xi, 0)-\Phi(\xi, 0) .
\end{aligned}
$$


Since the linear equation for $\Psi$ is asymptotically stable, and the forcing terms are in $E_{\mathbb{R}^{+}\left(\left(1+|\xi|^{j}\right) e^{-\gamma \xi}\right)}^{1}$ with norms bounded by $C\left(1+\tau^{k-j}\right)$, from Lemma 2.5 again,

$$
|\Psi|_{E_{\mathbb{R}^{+}}^{2}\left(\left(1+\xi^{j}\right) e^{-\gamma \xi}\right)} \leq C\left(1+\tau^{k-j}\right) .
$$

This proves the case $i=0$. The matching of $(S S)^{i}$ with $(R S)^{i+1}, 0 \leq i \leq r-1$, has been proved by induction. The matching of $(S S)^{i}$ with $(R S)^{i}, 1 \leq i \leq r$, can be treated similarly.

Theorem 6.4. Let $\Delta u_{j}^{i}=u_{j}^{S S i}-\tilde{u}_{j, 1}^{R S i}, 1 \leq i \leq r$, or $u_{j}^{S S i}-\tilde{u}_{j, 2}^{R S i}, 0 \leq i \leq r-1$, where $\tilde{u}_{j, 1}^{R S i}$ and $\tilde{u}_{j, 2}^{R S i}$ are defined in (6.8). Then there exists $\gamma>0$ such that for all integers $\alpha \leq 2, \beta \leq 1$,

$$
\left|\partial_{\xi}^{\alpha} \Delta u_{j}^{i}\right|+\left|\partial_{\tau}^{\beta} \Delta u_{j}^{i}\right| \leq C_{j}\left(1+|\xi|^{j}+\tau^{j}\right) e^{-\gamma|\xi|} .
$$

6.5. Constructing a pseudo solution. A pseudo solution is a piecewise smooth function that almost satisfies (1.1) with a small residual error

$$
\epsilon u_{t}-\epsilon^{2} u_{x x}-f(u, x, \epsilon),
$$

in the interior of each subregion where the function is smooth, and a small jump error at each of their common boundaries. By truncating the formal series, we can construct pseudo solutions with arbitrary accuracy.

Let

$$
\begin{array}{cc}
u^{R R i, m}(x, \epsilon)=\sum_{j=0}^{m} \epsilon^{j} u_{j}^{R R i}(x), & u^{S R i, m}(\xi, t, \epsilon)=\sum_{j=0}^{m} \epsilon^{j} u_{j}^{S R i}(\xi, t), \\
u^{R S i, m}(x, \tau, \epsilon)=\sum_{j=0}^{m} \epsilon^{j} u_{j}^{R S i}(x, \tau), & u^{S S i . m}(\xi, \tau, \epsilon)=\sum_{j=0}^{m} \epsilon^{j} u_{j}^{S S i}(\xi, \tau), \\
\eta^{i, m}(t, \epsilon)=\sum_{j=0}^{m} \epsilon^{j} \eta_{j}^{i}(t), & \eta^{*}{ }^{i, m}(\tau, \epsilon)=\sum_{j=0}^{m} \epsilon^{j} \stackrel{\eta}{j}_{j}^{i}(\tau) .
\end{array}
$$

We use $\eta^{i, m}$ and $\stackrel{*}{\eta}^{i, m}$ as abbreviations for $\eta^{i, m}(t, \epsilon)$ and $\stackrel{*}{\eta}^{i, m}(\tau, \epsilon)$. Let $0<\beta<1$ be a constant. Let the width of the initial and internal/boundary layers be $O\left(\epsilon^{\beta}\right)$. Define the subregions

$$
\begin{aligned}
& (R R)^{i, m}=\left\{t>\epsilon^{\beta}, \quad x \in\left(\eta^{i-1, m}+\epsilon^{\beta}, \eta^{i, m}-\epsilon^{\beta}\right)\right\}, \quad 1 \leq i \leq r, \\
& (S R)^{i, m}=\left\{t>\epsilon^{\beta}, \quad x \in\left(\eta^{i, m}-\epsilon^{\beta}, \eta^{i, m}+\epsilon^{\beta}\right) \cap[a, b]\right\}, \quad 0 \leq i \leq r, \\
& (R S)^{i, m}=\left\{0 \leq t<\epsilon^{\beta}, \quad x \in\left(\eta^{* i-1, m+1}+\epsilon^{\beta}, \eta^{*} \eta^{i, m+1}-\epsilon^{\beta}\right)\right\}, \quad 1 \leq i \leq r, \\
& (S S)^{i, m}=\left\{0 \leq t<\epsilon^{\beta}, \quad x \in\left(\eta^{* i, m+1}-\epsilon^{\beta}, \eta^{*} \eta^{i, m+1}+\epsilon^{\beta}\right)\right\}, \quad 0 \leq i \leq r .
\end{aligned}
$$

Theorem 6.5. For all $m \geq 0$, let

$$
U^{m}(x, t, \epsilon)= \begin{cases}u^{R R i, m}(x, t, \epsilon), & \text { if }(x, t) \in(R R)^{i, m}, \\ u^{S R i, m}\left(\frac{x-\eta^{i, m}}{\epsilon}, t, \epsilon\right), & \text { if }(x, t) \in(S R)^{i, m}, \\ u^{R S i, m}\left(x, \frac{t}{\epsilon}, \epsilon\right), & \text { if }(x, t) \in(R S)^{i, m}, \\ u^{S S i, m}\left(\frac{x-\eta^{i, m+1}}{\epsilon}, \frac{t}{\epsilon}, \epsilon\right), & \text { if }(x, t) \in(S S)^{i, m} .\end{cases}
$$


Then $U^{m}$ is a pseudo solution to (1.1) with residual error being $O\left(\epsilon^{\beta(m+1)}\right)$ in each layer where $U^{m}$ is smooth. The jump errors of $U^{m}, \partial_{t} U^{m}, \partial_{x} U^{m}$ and $\partial_{x}^{2} U^{m}$ are of $O\left(\epsilon^{\beta(m+1)}\right)$ along the common boundaries of adjacent layers. The function $U^{m}$ satisfies Neumann boundary conditions, and almost satisfies the initial condition with an error $U^{m}(x, 0, \epsilon)-\bar{u}(x, \epsilon)=O\left(\epsilon^{\beta(m+1)}\right)$.

Proof. The residual errors can be evaluated by substituting $U^{m}$ into (1.1) and expanding in powers of $\epsilon$. For example, in $(S S)^{i, m}$, the error is bounded by

$$
C \epsilon^{m+1} \sup \left\{1+|\xi|^{m+1}+|\tau|^{m+1}:|\xi|<\epsilon^{\beta-1},|\tau|<\epsilon^{\beta-1}\right\} \leq C \epsilon^{\beta(m+1)} .
$$

The estimates of jump errors use the matching of adjacent layers. For example, the jump between $(S S)^{i, m}$ and $(R S)^{i+1, m}$ at the boundary

$$
\begin{aligned}
\Gamma & =\left\{x=\eta^{* i, m+1}+\epsilon^{\beta}, 0 \leq \tau \leq \epsilon^{\beta-1}\right\} \\
& =\left\{\xi=\epsilon^{\beta-1}, 0 \leq \tau \leq \epsilon^{\beta-1}\right\},
\end{aligned}
$$

where $\xi=\frac{x-\eta^{*}, m+1}{\epsilon}$, is

$$
\begin{aligned}
& \left|\sum_{0}^{m} \epsilon^{j}\left(u_{j}^{S S i}(\xi, \tau)-u_{j}^{R S, i+1}(x, \tau)\right)\right| \\
& \quad \leq\left|\sum_{0}^{m} \epsilon^{j}\left(u_{j}^{S S i}(\xi, \tau)-\tilde{u}_{j, 2}^{R S i}(\xi, \tau)\right)\right|+\left|\sum_{0}^{m} \epsilon^{j}\left(\tilde{u}_{j, 2}^{R S i}(\xi, \tau)-u_{j}^{R S, i+1}(x, \tau)\right)\right| .
\end{aligned}
$$

The first term of the above is bounded by

$$
O\left(\sum_{0}^{m} \epsilon^{j} \sup \left\{\left(1+|\xi|^{j}+|\tau|^{j}\right) e^{-\gamma \xi}\right\}\right)=O\left(e^{-\gamma \epsilon^{\beta-1}}\right)
$$

where $\gamma>0$ is a constant, due to the matching condition Theorem 6.4. For the second term, observe that

$$
\begin{aligned}
\sum_{0}^{m} \epsilon^{j} u_{j}^{R S, m+1}\left(\epsilon \xi+\eta^{i, m+1}, \tau\right) & =\sum_{0}^{m} \epsilon^{j} u_{j}^{R S, m+1}\left(\epsilon \xi+\eta^{*}, \tau\right)+O\left(\epsilon^{\beta(m+1)}\right) \\
& =\sum_{0}^{m} \epsilon^{j} \tilde{u}_{j, 2}^{R S i}(\xi, \tau)+O\left(\epsilon^{\beta(m+1)}\right) .
\end{aligned}
$$

Here the $O\left(\epsilon^{\beta(m+1)}\right)$ terms are caused by truncating $\stackrel{*}{\eta}^{i}$. This gives the desired estimate on the jump error. The other jump errors can be estimated similarly.

Finally we mention that using composite expansion techniques we can construct pseudo solutions with only residual error but no jump error in the entire region $t \geq 0, x \in[a, b]$. See [22] and $[6,7]$ for more details.

\section{Proof of the Lemmas}

Proof of Lemma 2.2. Consider a system in $\mathbb{R}^{2 n}$ that is equivalent to (2.9):

$$
\begin{aligned}
& u_{\xi}=v \\
& v_{\xi}=-D f(p) u-V v .
\end{aligned}
$$


Let $\lambda$ be an eigenvalue for $J=\left(\begin{array}{cc}0 & I \\ -D f(p) & -V\end{array}\right)$. Then,

$$
\operatorname{det}(\lambda I-J)=\prod_{i=1}^{n}\left(\lambda^{2}+V \lambda+\mu_{i}\right)
$$

where $\mu_{i}, 1 \leq i \leq n$, are the eigenvalues for $D f(p)$. Since $R e \mu_{i} \leq-\sigma_{0}$, it is elementary to show that $\lambda^{2}+V \lambda+\mu_{i}=0$ has two roots $\left\{\lambda_{i 1}, \lambda_{i 2}\right\}$ with $\operatorname{Re} \lambda_{i 1} \leq$ $-V-\sqrt{V^{2}+4 \sigma_{0}}<\sqrt{V^{2}+4 \sigma_{0}}-V \leq \operatorname{Re} \lambda_{i 2}$. Therefore (7.1) has exponential dichotomy with stable and unstable spaces both $n$-dimensional, so does (2.9).

Since $D f(q(\xi)) \rightarrow D f\left(p^{i}\right), i=1$ or 2 as $\xi \rightarrow-\infty$ or $+\infty$, by a perturbation theory of exponential dichotomy, cf. [26], (2.10) has exponential dichotomies on $R^{-}$and $\mathbb{R}^{+}$respectively. The stable and unstable spaces, $\mathcal{R} P_{s}(t)$ and $\mathcal{R} P_{u}(t)$ are $n$-dimensional. The rate of decay on $\mathcal{R} P_{s}(t)$ and $\mathcal{R} P_{u}(t)$ can be any constant $0<\alpha_{1}<\alpha$. Since $\alpha$ can be arbitrary close to $\sqrt{V^{2}+4 \sigma_{0}}-|V|$, so is $\alpha_{1}$.

We now consider the following systems

$$
\begin{aligned}
u_{\xi} & =v, \\
u_{\xi} & =-V v-D f(p) u+g, \\
u_{\xi} & =v, \\
v_{\xi} & =-V v-\operatorname{Df}(q(\xi)) u+g .
\end{aligned}
$$

Let $T(\xi, s)$ be the solution operator for $(7.3)$.

Proof of Lemma 2.3. (i) Let $g \in X=E_{\mathbb{R}}^{m}(w)$. Let $L_{p} u=u_{\xi \xi}+V u_{\xi}+D f(p) u$, with $D\left(L_{p}\right)=E_{\mathbb{R}}^{m+2}(w)$. From Lemma 2.2, system (7.2) that is equivalent to $L_{p} u=g$ has an exponential dichotomy on $\mathbb{R}$. Let $P_{u}$ and $P_{s}$ be the projection to the unstable and stable spaces. Let

$$
\left(\begin{array}{l}
u \\
v
\end{array}\right)(\xi)=\int_{-\infty}^{\xi} e^{J(\xi-s)} P_{s}\left(\begin{array}{c}
0 \\
g(s)
\end{array}\right) d s+\int_{\infty}^{\xi} e^{J(\xi-s)} P_{u}\left(\begin{array}{l}
0 \\
g(s)
\end{array}\right) d s .
$$

Using the exponential estimates on $\left\|e^{J(\xi-s)^{\infty}} P_{s}\right\|$ and $\left\|e^{J(\xi-s)} P_{u}\right\|$, and Lemma 2.1, we can verify that $(u, v) \in E_{\mathbb{R}}^{m+2}(w) \times E_{\mathbb{R}}^{m+1}(w)$ and $(u, v)$ solves (7.2). Details will be omitted. Therefore $\mathcal{R}\left(L_{p}\right)=X$. On the other hand, if $u$ is a solution to $L_{p} u=g$, then $\left(u, u_{\xi}\right) \in E_{\mathbb{R}}^{m+2}(w) \times E_{\mathbb{R}}^{m+1}(w)$ is a solution to (7.2). It is standard to show $\left(u, u_{\xi}\right)$ is given by (7.4). This proves that $\operatorname{Ker}\left(L_{p}\right)=\{0\}$. by

(ii) Let $g \in X=E_{\mathbb{R}^{+}}^{m}(w)$. All the solutions of (7.3) with $u \in E_{\mathbb{R}^{+}}^{m+2}(w)$ are given

$$
\begin{aligned}
\left(\begin{array}{l}
u \\
v
\end{array}\right)(\xi) & =T(\xi, 0)\left(\begin{array}{l}
u_{0} \\
v_{0}
\end{array}\right)+\int_{0}^{\xi} T(\xi, s) P_{s}\left(\begin{array}{l}
0 \\
g(s)
\end{array}\right) d s \\
& +\int_{\infty}^{\xi} T(\xi, s) P_{u}\left(\begin{array}{c}
0 \\
g(s)
\end{array}\right) d s,
\end{aligned}
$$

where $\left(u_{0}, v_{0}\right) \in \mathcal{R} P_{s}(0)$. Let $\Pi(u, v)=v$ be the projection from $\mathbb{R}^{2 n} \sim \mathbb{R}^{n} \times \mathbb{R}^{n}$ to $\mathbb{R}^{n}$. We can show that $\Pi: \mathcal{R} P_{s} \rightarrow \mathbb{R}^{n}$ is a homeomorphism. If not, then there exists a nontrivial $\left(\begin{array}{l}u_{0} \\ v_{0}\end{array}\right) \in \mathcal{R} P_{s}(0)$, such that $v_{0}=0$. Thus, there exists a nontrivial 
bounded solution to $(2.10)$ with $u_{\xi}(0)=0$. This is a contradiction. Based on what have been proved, there exists a unique $\left(\begin{array}{c}u_{0} \\ v_{0}\end{array}\right) \in \mathcal{R} P_{s}$ such that

$$
\Pi\left(\begin{array}{c}
u_{0} \\
v_{0}
\end{array}\right)=v_{0}=\phi-\Pi \int_{\infty}^{0} T(0, s) P_{u}\left(\begin{array}{c}
0 \\
g(s)
\end{array}\right) d s .
$$

The desired solution can be obtained by such $\left(\begin{array}{c}u_{0} \\ v_{0}\end{array}\right)$.

The case $X=E_{\mathbb{R}^{-}}^{m}(w)$ can be treated similarly.

Finally the estimates on $\|u\|_{E^{m+2}(w)}$ in both cases (i) and (ii) come from Banach's closed graph theorem.

Proof of Lemma 2.4. Consider (7.3) but with $\xi \in \mathbb{R}, g \in X=E_{\mathbb{R}}^{m}(w)$. Equation $L_{q} u=g$ is then equivalent to (7.3). From Lemma 2.2, (7.3) has exponential dichotomies on $\mathbb{R}^{+}$and $\mathbb{R}^{-}$. Also if $g=0,\left(q_{\xi}, q_{\xi \xi}\right)$ is the only bounded solution to (7.3), up to constant multiples. It follows from the same argument as in [26], which treats the case $w \equiv 1$, that $(7.3)$ has a solution $(u, v) \in E_{\mathbb{R}}^{m+2}(w) \times E_{\mathbb{R}}^{m+1}(w)$ if and only if $\left\langle\left(\begin{array}{l}0 \\ g\end{array}\right),\left(\begin{array}{l}\psi_{1} \\ \psi_{2}\end{array}\right)\right\rangle_{L^{2}}=0$, where $\left(\psi_{1}, \psi_{2}\right)$ is a unique (up to constant multiples) bounded solution to the adjoint equation of (7.3)

$$
\begin{aligned}
& \psi_{1 \xi}=D f^{\tau}(q(\xi)) \psi_{2}, \\
& \psi_{2 \xi}=-\psi_{1}+V \psi_{2} .
\end{aligned}
$$

It is now clear that $\left\langle g, \psi_{2}\right\rangle=0 \Leftrightarrow g \in \mathcal{R}\left(L_{q}\right)$. The equation for $\psi_{2}$ is $\psi_{2 \xi \xi}-V \psi_{2 \xi}+$ $D f^{\tau}(q(\xi)) \psi_{2}=0$.

Proof of Lemma 2.5. Using the definition of the exponential stability right before Lemma 2.5, we have

$$
\begin{aligned}
\left\|T(t, 0) u_{0}\right\|_{\theta} & \leq K e^{-\alpha t}\left\|u_{0}\right\|_{\theta} \leq C \\
\left\|\int_{0}^{t} T(t, s) g(s) d s\right\|_{\theta} & \leq \int_{0}^{t}\left\|T\left(t, \frac{t+s}{2}\right) T\left(\frac{t+s}{2}, s\right) g(s)\right\|_{\theta} d s \\
& \leq \int_{0}^{t} K e^{-\alpha(t-s) / 2} K\left(1+\left(\frac{t-s}{2}\right)^{\beta-\theta}\right) \cdot C\left(1+s^{k}\right) d s \\
& \leq C\left(1+t^{k}\right) .
\end{aligned}
$$

The desired estimate of $u(t)$ follows from the variation of constant formula.

Proof of Lemma 2.6. For any $\gamma \in \mathbb{R}$, the locus of $\mathcal{P}=\left\{\lambda^{2}|\operatorname{Re} \lambda=2| \gamma \mid+1\right\}$ is a parabola. Let $\Sigma=\left\{\left|\arg \left(\lambda-\sigma_{1}\right)\right|<\frac{\pi}{2}+\delta\right\}, 0<\delta<\frac{\pi}{2}$, be a sector. Let $\sigma_{1}>0$ be sufficiently large such that $\Sigma \cap \mathcal{P}=\emptyset$. Then $\lambda \in \Sigma$ implies that $\operatorname{Re} \sqrt{\lambda}>2|\gamma|+1$, where $\sqrt{\lambda}$ is in the branch with $|\arg \sqrt{\lambda}|<\pi / 2$.

(i) Let $g \in X=E_{\mathbb{R}}(w), w=\left(1+|\xi|^{j}\right) e^{-\gamma \xi}, \lambda \in \Sigma$. Consider $u_{\xi \xi}-\lambda u=g$, and its equivalent system

$$
\begin{aligned}
u_{\xi} & =\sqrt{\lambda} v \\
v_{\xi} & =\sqrt{\lambda} u+g / \sqrt{\lambda} .
\end{aligned}
$$


The eigenvalues for $H=\left(\begin{array}{cc}0 & \sqrt{\lambda} I \\ \sqrt{\lambda} I & 0\end{array}\right)$ are $\mu= \pm \sqrt{\lambda}$, each is of multiplicity $n$. Also $|R e \mu|>2|\gamma|+1$. Therefore (7.7) has an exponential dichotomy on $\mathbb{R}$, with $n$-dimensional stable and unstable subspaces.

For the matrix $H, \mathcal{R} P_{u}=\left\{\left(\begin{array}{c}w \\ w\end{array}\right) \mid w \in \mathbb{R}^{n}\right\}$ that is associated to eigenvalue $\sqrt{\lambda}$ and $\mathcal{R} P_{s}=\left\{\left(\begin{array}{c}w \\ -w\end{array}\right) \mid w \in \mathbb{R}^{n}\right\}$ that is associated to eigenvalue $-\sqrt{\lambda}$. Thus, $P_{s}\left(\begin{array}{c}u \\ v\end{array}\right)=\left(\begin{array}{c}(u-v) / 2 \\ (v-u) / 2\end{array}\right)$ and $P_{u}\left(\begin{array}{c}u \\ v\end{array}\right)=\left(\begin{array}{c}(u+v) / 2 \\ (u+v) / 2\end{array}\right)$ with $\left|P_{u}\right|+\left|P_{s}\right| \leq C$. The constant $C$ is independent of $\lambda \in \Sigma$. Thus, in the following, the constant $K$ is independent of $\lambda \in \Sigma$.

$$
\begin{array}{ll}
\left|e^{H \xi} P_{s}\right| \leq K e^{-R e \sqrt{\lambda} \xi}, \quad \xi \geq 0 \\
\left|e^{H \xi} P_{u}\right| \leq K e^{R e \sqrt{\lambda} \xi}, \quad \xi \leq 0 .
\end{array}
$$

Using (7.8), we can show that the integrals in (7.9) converge and define a solution to $(7.7)$.

$$
\left(\begin{array}{l}
u \\
v
\end{array}\right)(\xi)=\int_{-\infty}^{\xi} e^{H(\xi-s)} P_{s}\left(\begin{array}{c}
0 \\
g(s) / \sqrt{\lambda}
\end{array}\right) d s+\int_{\infty}^{\xi} e^{H(\xi-s)} P_{u}\left(\begin{array}{l}
0 \\
g(s) / \sqrt{\lambda}
\end{array}\right) d s .
$$

In fact, using the estimates (7.8) and Lemma 2.1, we have

$$
\begin{aligned}
\left|\left(\begin{array}{c}
u \\
v
\end{array}\right)(\xi)\right| & \leq \int_{-\infty}^{\xi} K e^{-\operatorname{Re} \sqrt{\lambda}(\xi-s)}\|g\|_{E(w)} \cdot \frac{1}{|\sqrt{\lambda}|}\left(1+|s|^{j}\right) e^{-\gamma s} d s \\
& +\int_{\xi}^{\infty} K e^{-\operatorname{Re} \sqrt{\lambda}(s-\xi)}\|g\|_{E(w)} \frac{1}{|\sqrt{\lambda}|}\left(1+|s|^{j}\right) e^{-\gamma s} d s \\
& \leq \frac{K_{1}\|g\|_{E(w)}}{(\operatorname{Re} \sqrt{\lambda}-|\gamma|)^{j+1} \cdot|\sqrt{\lambda}|} \cdot\left(1+|\xi|^{j}\right) e^{-\gamma \xi}
\end{aligned}
$$

Here $K_{1}$ is a constant that depends on $j$. From $\operatorname{Re} \sqrt{\lambda}>2|\gamma|+1$, we have $\operatorname{Re} \sqrt{\lambda}-$ $|\gamma|>1$ and $\operatorname{Re} \sqrt{\lambda}-|\gamma|>\frac{1}{2} \operatorname{Re} \sqrt{\lambda}$. Therefore the above is bounded by

$$
\frac{2 K_{1}\|g\|_{E(w)}}{\operatorname{Re} \sqrt{\lambda} \cdot|\sqrt{\lambda}|} \cdot\left(1+|\xi|^{j}\right) e^{-\gamma \xi}
$$

Since $\sigma_{1}>0,|\arg \lambda| \leq\left|\arg \left(\lambda-\sigma_{1}\right)\right| \leq \frac{\pi}{2}+\delta$, and $|\arg \sqrt{\lambda}| \leq \frac{\pi}{4}+\frac{\delta}{2}<\frac{\pi}{2}$. Thus, $\operatorname{Re} \sqrt{\lambda}>|\sqrt{\lambda}| \cos \left(\frac{\pi}{4}+\frac{\delta}{2}\right)=C_{0}|\sqrt{\lambda}|$. From this

$$
\left|\left(\begin{array}{c}
u \\
v
\end{array}\right)(\xi)\right| \leq \frac{2 K_{1}\|g\|_{E(w)}}{C_{0}|\lambda|}\left(1+|\xi|^{j}\right) e^{-\gamma \xi}
$$

Thus $\|u\|_{E(w)} \leq \frac{c}{|\lambda|}\|g\|_{E(w)} \leq \frac{c}{\left|\lambda-\sigma_{1}\right|}\|g\|_{E(w)}$. The solution in (7.9) is also unique in $E_{\mathbb{R}}(w)$ since (7.7) has an exponential dichotomy on $\mathbb{R}$. This proves the Lemma when $X=E_{\mathbb{R}}(w)$.

(ii) Let $g \in X=E_{\mathbb{R}^{+}}(w), \lambda \in \Sigma$. Consider $u_{\xi \xi}-\lambda u=g$ with the boundary condition $u_{\xi}(0)=0$. If $\left(\begin{array}{c}u \\ v\end{array}\right) \in E_{\mathbb{R}^{+}}^{m}(w)$ is a solution for (7.7), it can be expressed 
as

$$
\begin{aligned}
\left(\begin{array}{l}
u \\
v
\end{array}\right)(\xi) & =e^{H \xi}\left(\begin{array}{c}
u_{0} \\
v_{0}
\end{array}\right)+\int_{0}^{\xi} e^{H(\xi-s)} P_{s}\left(\begin{array}{c}
0 \\
g(s) / \sqrt{\lambda}
\end{array}\right) d s \\
& +\int_{\infty}^{\xi} e^{H(\xi-s)} P_{u}\left(\begin{array}{c}
0 \\
g(s) / \sqrt{\lambda}
\end{array}\right) d s
\end{aligned}
$$

Here $\left(\begin{array}{l}u_{0} \\ v_{0}\end{array}\right) \in \mathcal{R} P_{s}$ has to be determined so that $v(0)=0$. To this end, choose

$$
v_{0}=-\Pi \int_{\infty}^{0} e^{-H s} P_{u}\left(\begin{array}{c}
0 \\
g(s) / \sqrt{\lambda}
\end{array}\right) d s
$$

and $u_{0}=-v_{0}$, then $\left(\begin{array}{c}u_{0} \\ v_{0}\end{array}\right) \in \mathcal{R} P_{s}$. Similar to (i), $\left|\left(\begin{array}{c}u_{0} \\ v_{0}\end{array}\right)\right|_{\mathbb{R}^{2 n}} \leq C\|g\|_{E_{\mathbb{R}}^{+}(w)} /|\lambda|$. With this $\left(\begin{array}{l}u_{0} \\ v_{0}\end{array}\right)$, we have

$$
\left|\left(\begin{array}{c}
u \\
v
\end{array}\right)\right|_{E_{\mathbb{R}^{+}}(w)} \leq C\|g\|_{E(w) /|\lambda|} \leq C\|g\|_{E_{\mathbb{R}^{+}}(w)} /\left|\lambda-\sigma_{1}\right| .
$$

This proves the case $X=E_{\mathbb{R}^{+}}(w)$. The same argument can be used for the case $X=E_{\mathbb{R}^{-}}(w)$.

(iii) Let $g \in X=B_{\mathbb{R}}(w), \lambda \in \Sigma$. If $\left(\begin{array}{c}u \\ v\end{array}\right) \in B_{\mathbb{R}}(w)$ is a solution to (7.7), it is expressed by (7.9). Let $g=g(\infty)+g_{1}(\xi)$, where $g(\infty)=\lim _{\xi \rightarrow \infty} g(\xi)$. Then $g_{1} \in E_{\mathbb{R}}(w)$. It follows that $\left(\begin{array}{l}u \\ v\end{array}\right)=\left(\begin{array}{l}u_{1} \\ v_{1}\end{array}\right)+\left(\begin{array}{l}u_{2} \\ v_{2}\end{array}\right)$, where $\left(\begin{array}{c}u_{2} \\ v_{2}\end{array}\right)=\left(\begin{array}{c}-g(\infty) / \lambda \\ 0\end{array}\right)$ is a constant solution and

$$
\left(\begin{array}{l}
u_{1} \\
v_{1}
\end{array}\right)(\xi)=\int_{-\infty}^{\xi} e^{H(\xi-s)} P_{s}\left(\begin{array}{c}
0 \\
g_{1}(s) / \sqrt{\lambda}
\end{array}\right) d s+\int_{\infty}^{\xi} e^{H(\xi-s)} P_{u}\left(\begin{array}{c}
0 \\
g_{1}(s) / \sqrt{\lambda}
\end{array}\right) d s .
$$

From (i), $\left\|u_{1}\right\|_{E_{\mathbb{R}}(w)} \leq C\left\|g_{1}\right\|_{E_{\mathbb{R}}(w) /|\lambda|}$. Also, $\left|u_{2}\right| \leq|g(\infty)| /|\lambda|$. Thus, $\|u\|_{B_{\mathbb{R}}(w)} \leq$ $C\|g\|_{B_{\mathbb{R}}(w)} /|\lambda| \leq C\|g\|_{B_{\mathbb{R}}}(w) /\left|\lambda-\sigma_{1}\right|$. This proves the case $X=B_{\mathbb{R}}(w)$. The cases $X=B_{\mathbb{R}^{ \pm}}(w)$ can be handled similar to (ii).

Proof of Lemma 2.7. (i) Let $w=1+|\xi|^{j}, X=E_{\mathbb{R}}(w)$ and $D_{A}=E_{\mathbb{R}}^{2}(w)$ first. Let $u_{0} \in E_{\mathbb{R}}^{1}(w), v=D_{\xi} u_{0} \in E_{\mathbb{R}}(w)$. Let

$$
I(t)=t^{1 / 2} A e^{A t} u_{0}=t^{1 / 2} D_{\xi}^{2} e^{A t} u_{0}=t^{1 / 2} D_{\xi} e^{A t} v .
$$

Using the fundamental solution for the heat equation

$$
\begin{aligned}
I(\xi, t) & =t^{1 / 2} D_{\xi} \int_{-\infty}^{\infty}(2 \sqrt{\pi t})^{-1} \exp \left(-\frac{x^{2}}{4 t}\right) v(\xi-x) d x \\
& =(2 \sqrt{\pi})^{-1} \int_{-\infty}^{\infty} \exp \left(-\frac{x^{2}}{4 t}\right) v(\xi-x)\left(-\frac{2 x}{4 t}\right) d x
\end{aligned}
$$


Let $\frac{x^{2}}{4 t}=\eta, x= \pm \sqrt{4 t \eta}$.

$$
\begin{aligned}
|2 \sqrt{\pi} I(\xi, t)| \leq \mid & -\int_{\infty}^{0} e^{-\eta} v(\xi+\sqrt{4 t \eta}) d \eta \\
& -\int_{0}^{\infty} e^{-\eta} v(\xi-\sqrt{4 t \eta}) d \eta \mid \\
= & \mid \int_{0}^{\infty} e^{-\eta}[v(\xi+\sqrt{4 t \eta})-v(\xi)] d \eta . \\
& -\int_{0}^{\infty} e^{-\eta}[v(\xi-\sqrt{4 t \eta})-v(\xi)] d \eta \mid \\
\left|2 \sqrt{\pi}\left(1+|\xi|^{j}\right)^{-1} I(\xi, t)\right| \leq & \left|\int_{0}^{M}+\int_{M}^{\infty} e^{-\eta}\right| v(\xi+\sqrt{4 t \eta})-v(\xi)\left|\left(1+|\xi|^{j}\right)^{-1} d \eta\right| \\
+ & \left|\int_{0}^{M}+\int_{M}^{\infty} e^{-\eta}\right| v(\xi-\sqrt{4 t \eta})-v(\xi)\left|\left(1+|\xi|^{j}\right)^{-1} d \eta\right| \\
= & I_{1}(\xi, t)+I_{2}(\xi, t) .
\end{aligned}
$$

We show $\lim _{t \rightarrow 0^{+}} \sup _{\xi} I_{1}(\xi, t)=0$. Similar arguments will show $\lim _{t \rightarrow 0^{+}} \sup _{\xi} I_{2}(\xi, t)=0$, thus $\lim _{t \rightarrow 0^{+}}|I(t)|_{E(w)}=0$. For any $\epsilon>0$, choose $M$ so large such that

$$
\begin{array}{r}
\int_{M}^{\infty} e^{-\eta}|v(\xi)|\left(1+|\xi|^{j}\right)^{-1} d \eta \leq \int_{M}^{\infty} e^{-\eta} d \eta \cdot|v|_{E(w)}<\frac{\epsilon}{4} \\
\int_{M}^{\infty} e^{-\eta}|v(\xi+\sqrt{4 t \eta})|\left(1+|\xi+\sqrt{4 t \eta}|^{j}\right)^{-1} \frac{1+|\xi+\sqrt{4 t \eta}|^{j}}{1+|\xi|^{j}} d \eta \\
\leq C \int_{M}^{\infty} e^{-\eta}|v|_{E(w)}\left(1+(\sqrt{4 t \eta})^{j}\right) d \eta<\frac{\epsilon}{4},
\end{array}
$$

For that fixed $M$, let $t_{\epsilon}$ be small such that for $0<t<t_{\epsilon}$,

$$
\int_{0}^{M} e^{-\eta}\left|v(\xi+\sqrt{4 t \eta})\left(1+|\xi+\sqrt{4 t \eta}|^{j}\right)^{-1}-v(\xi)\left(1+|\xi|^{j}\right)^{-1}\right| d \eta<\frac{\epsilon}{4} .
$$

This is possible since $v(\xi) w^{-1}(\xi)$ is uniformly continuous. Also if $t_{\epsilon}$ is even smaller, then

$$
\begin{gathered}
\int_{0}^{M} e^{-\eta}|v(\xi+\sqrt{4 t \eta})|\left|\left(1+|\xi+\sqrt{4 t \eta}|^{j}\right)^{-1}-\left(1+|\xi|^{j}\right)^{-1}\right| d \eta \\
\quad \leq \int_{0}^{M} e^{-\eta}|v|_{E(w)}\left(1-\frac{1+|\xi+\sqrt{4 t \eta}|^{j}}{1+|\xi|^{j}}\right) d \eta<\frac{\epsilon}{4},
\end{gathered}
$$

since $\frac{|\xi+\alpha|^{j}-|\xi|^{j}}{1+|\xi|^{j}} \rightarrow 0$ uniformly with respect to $\xi$ as $\alpha \rightarrow 0$.

From (7.10)-(7.13), we see $\lim _{t \rightarrow 0^{+}} \sup _{\xi} I_{1}(\xi, t)=0$.

(ii) Let $X=E_{\mathbb{R}^{+}}(w), D_{A}=E_{\mathbb{R}^{+}}^{2}(w) \cap B C$, and $u_{0} \in E_{\mathbb{R}^{+}}^{1}(w) \cap B C$. Let $\tilde{u}_{0}$ be the even extension of $u_{0}$ to $\xi \in \mathbb{R}$. Then $\tilde{u}_{0} \in E_{\mathbb{R}}(w)$. To find $e^{A t} u_{0}$, we solve

$$
\tilde{u}_{t}=u_{\xi \xi}, \quad \tilde{u}(0)=\tilde{u}_{0} .
$$


The restriction of $\tilde{u}(t)$ to $\xi \geq 0$ is $e^{A t} u_{0}$. Using the results from (i), we find that $\left|t^{1 / 2} A e^{A t} u_{0}\right|_{E_{\mathbb{R}^{+}}(w)} \leq\left|t^{1 / 2} D_{\xi}^{2} \tilde{u}(t)\right|_{E_{\mathbb{R}}(w)}$. The latter approaches 0 as $t \rightarrow 0^{+}$. Thus $u_{0} \in D_{A}\left(\frac{1}{2}\right)$.

(iii) The other cases can be treated similarly.

Proof of Lemma 2.9. (a) Let $\lambda \in \mathbb{C}$ be such that Re $\lambda>-\sigma_{0}$. Let $g \in X$ and consider $(L-\lambda) u=g$. The equivalent system is

$$
\begin{aligned}
& u_{\xi}=v \\
& v_{\xi}=(\lambda I-M) u-V v+g .
\end{aligned}
$$

Let $J=\left(\begin{array}{ll}0 & I \\ \lambda I-M & -V I\end{array}\right)$. Since $\operatorname{Re} \sigma(\lambda I-M)>0$, it can be verified that $J$ is hyperbolic with $n$-dimensional stable and unstable subspaces. Assume that $\eta+i \omega \in \sigma(\lambda I-M)$, and $\mu \in \sigma J$. Then $\mu=\left[-V \pm \sqrt{V^{2}+4 \eta+4 i \omega}\right] / 2$. One can verify that $R e \sqrt{V^{2}+4 \eta+4 i \omega}>\operatorname{Re} \sqrt{V^{2}+4 \eta}$. Thus, $|R e \mu|>\sqrt{V^{2}+4 \eta}-|V|$. If $\operatorname{Re} \lambda+\sigma_{0}>\eta_{0}$ for some $\eta_{0}>0$, then let $\gamma_{0}=\sqrt{V^{2}+4 \eta_{0}}-|V|$, we have

$$
\begin{array}{ll}
\left|e^{J \xi} P_{s}\right|<K e^{-\gamma_{0} \xi}, & \xi \geq 0, \\
\left|e^{J \xi} P_{u}\right|<K e^{\gamma_{0} \xi}, & \xi \leq 0,
\end{array}
$$

for some $K>0$, where $P_{s}$ and $P_{u}$ are the projections to stable and unstable subspaces of $\mathbb{R}^{2 n}$. Therefore, if $\gamma$ is the constant used to define $w$, with $|\gamma|<\gamma_{0}$,

$$
\left(\begin{array}{c}
u \\
v
\end{array}\right)(\xi)=\int_{-\infty}^{\xi} e^{J(\xi-\eta)} P_{s}\left(\begin{array}{c}
0 \\
g(\eta)
\end{array}\right) d_{\eta}+\int_{\infty}^{\xi} e^{J(\xi-\eta)} P_{u}\left(\begin{array}{c}
0 \\
g(\eta)
\end{array}\right) d \eta
$$

is the unique solution to (7.14) that is in $E_{\mathbb{R}}^{2}(w)$. It is easy to show that $|u|_{E^{2}(w)} \leq$ $C|g|_{E(w)}$. Thus, $\lambda \in \rho(L)$. This proves the case $X=E_{\mathbb{R}}(w)$.

Now consider $X=E_{\mathbb{R}^{ \pm}}(w)$ and $g \in X$. Replace $g$ by its even extension $\tilde{g}$ and consider (7.14). The solution $\left(\begin{array}{l}u \\ v\end{array}\right)$ can be expressed by (7.15) and is even in $\xi \in \mathbb{R}$. Consider its restriction on $\xi \in \mathbb{R}^{ \pm}$, then $u \in E_{\mathbb{R}^{ \pm}}^{2}(w) \cap B C$.

(b) From part (a), if $\operatorname{Re} \lambda>-\sigma_{0}+\eta_{0}$, equation $u_{\xi \xi}+V u_{\xi}+D f\left(p^{i}\right) u-\lambda u=0$ has exponential dichotomies on $\mathbb{R}^{-}$and $\mathbb{R}^{+}$. Therefore

$$
\begin{aligned}
& u_{\xi}=v, \\
& v_{\xi}=[\lambda I-D f(q)] u-V v
\end{aligned}
$$

has exponential dichotomies on $\mathbb{R}^{-}$and $\mathbb{R}^{+}$with projections $P_{s}(t)+P_{u}(t)=I, t \in$ $\mathbb{R}^{-}$and $\mathbb{R}^{+}$respectively. If $\lambda$ is such that $\mathbb{R} P_{u}\left(0^{-}\right) \cap \mathbb{R} P_{s}\left(0^{+}\right)=\{0\}$, then (7.16) has exponential dichotomy on $\mathbb{R}$. Similar to part (a), we can show $\lambda \in \rho\left(L_{q}\right)$. If $\lambda$ is such that $\mathcal{R} P_{u}\left(0^{-}\right) \cap \mathcal{R} P_{s}\left(0^{+}\right) \neq\{0\}$, then $\lambda$ is an eigenvalue of finite multiplicity.

Proof of Lemma 4.1. (i) P1 comes from H1 by the implicit function theorem and the continuous dependence of eigenvalues on the parameter $x$.

(ii) Equation (4.4) has a heteroclinic solution $q(\xi)$ when $x=x^{i}$ and $V^{i}\left(x^{i}\right)=0$. Melnikov's method is used to determine the existence of heteroclinic solutions $(u, v)$ near $\left(q, q_{\xi}\right)$ for the following system

$$
\begin{aligned}
& u_{\xi}=v \\
& v_{\xi}=-f_{0}(u, x)-V v .
\end{aligned}
$$


Here $V \in \mathbb{R}$ and $x$ are parameters. Based on H1, H2 and Lemma 2.2, the linear variational equations for (7.17) around $(u, v)=\left(q, q_{\xi}\right)$ has exponential dichotomies on $\mathbb{R}^{-}$and $\mathbb{R}^{+}$with $\mathcal{R} P_{s}\left(0^{+}\right) \cap \mathcal{R} P_{u}\left(0^{-}\right)$spanned by $\left\{\left(q_{\xi}(0), q_{\xi \xi}(0)\right)\right\}$, when $V=0$ and $x=x^{i}$. Let $G(x, V)$ denote the function that measures the distance between $W^{u}\left(p^{1}\right)$ and $W^{s}\left(p^{2}\right)$ along the direction $\left(\psi_{i 1}(0),, \psi_{2 i}(0)\right)$ based on the point $\left(q(0), q_{\xi}(0)\right)$. cf. (7.6). Notice that $\left(\psi_{i 1}(0), \psi_{2 i}(0)\right)$ is transverse to $T_{q(0)} W^{u}\left(p^{\prime}\right)+T_{q(0)} W^{s}\left(p^{2}\right)$, thus, $G(x, V)$ is well defined. From [26],

$$
\begin{aligned}
& \frac{\partial G\left(x^{i}, 0\right)}{\partial x}=\int_{-\infty}^{\infty} \psi_{i 2}^{\tau}(\xi) f_{0 x}\left(q^{i}(\xi), x^{i}\right) d \xi \\
& \frac{\partial G\left(x^{i}, 0\right)}{\partial V}=\int_{-\infty}^{\infty} \psi_{i 2}^{\tau}(\xi) q_{\xi}^{i}(\xi) d \xi .
\end{aligned}
$$

From (4.1), $\frac{\partial G\left(x^{i}, 0\right)}{\partial V} \neq 0$. Therefore there exist $V^{i}(x)$ such that $G\left(x, V^{i}(x)\right)=0$ for $x$ in a neighborhood of $x^{i}$. The method in [26] also insures that the heteroclinic solution $q^{i}(\xi, x)$ depends smoothly on $x$.

(iii) Define $L_{x}^{i} \varphi$ by the left hand side of (4.5). When $x=x^{i}, L_{x}^{i}: C_{b u}^{2} \rightarrow C_{b u}^{0}$ is Fredholm with index equal to zero, see [26]. From [30, page 115], if $x$ is in a small neighborhood of $x^{i}, L_{x}^{i}$ is Fredholm with index zero. Also $\operatorname{dim} \mathcal{K} L_{x}^{i} \leq \operatorname{dim} \mathcal{K} L_{x^{i}}^{i}$. Since $q_{\xi} \in \mathcal{K} L_{x}^{i}$, we have $\operatorname{dim} \mathcal{K} L_{x}^{i}=1$ for all $x \in O^{i}$. Therefore (4.6) has a unique bounded solution $\psi_{i}(\xi, x)$ up to constant multiples.

It remains to show that by choosing $\left|\psi_{i}(\xi, x)\right|=1, \psi_{i}$ is a smooth function of $x$. Let $\left\{U_{1}=\left(q_{\xi}(0), q_{\xi \xi}(0)\right), U_{2}, \ldots, U_{n}\right\}$ be an orthogonal basis for $\mathcal{R} P_{u}\left(0^{-}\right)$and $\left\{\bar{U}_{1}=U_{1}, \bar{U}_{2}, \ldots, \bar{U}_{n}\right\}$ be an orthogonal basis for $\mathcal{R} P_{s}\left(0^{+}\right)$of the system

$$
\begin{aligned}
& u_{\xi}=v, \\
& v_{\xi}=f_{0 u}\left(q^{i}(\xi, x), x\right) u-V^{i}(x) v,
\end{aligned}
$$

when $x=x^{i}$ and $V^{i}(x)=0$. System (7.19) has exponential dichotomies on $\mathbb{R}^{-}$and $\mathbb{R}^{+}$when $x \in O^{i}$. Let $P_{u}(x, t)$ and $P_{s}(x, t)$ denote the projections to the unstable and stable spaces. $P_{u}\left(x^{i}, t\right)=P_{u}(t)$ and $P_{s}\left(x^{i}, t\right)=P_{s}(t)$.

Assume that $x$ is near $x^{i}$ so that $V^{i}(x)$ is near zero. For each $U_{i}, 2 \leq i \leq n$, there exists a unique $\Delta U_{i} \in \mathcal{R} P_{s}\left(0^{-}\right)$such that $U_{i}+\Delta U_{i} \in \mathcal{R} P_{u}\left(x, 0^{-}\right)$. Also for each $\bar{U}_{i}, 2 \leq i \leq n$, there exists a unique $\Delta \bar{U}_{i} \in \mathcal{R} P_{u}\left(0^{+}\right)$such that $\bar{U}_{i}+\Delta \bar{U}_{i} \in$ $\mathcal{R} P_{s}\left(x, 0^{+}\right)$. The functions $\Delta U_{i}$ and $\Delta \bar{U}_{i}$ are smooth functions of $x$. See [18] for details. For $i=1$ let $U_{1}+\Delta U_{1}=\left(q_{\xi}^{i}(0, x), q_{\xi \xi}^{i}(0, x)\right)$. $\Delta U_{1}$ is also smooth in $x$. In particular, $\left|\Delta U_{i}\right|+\left|\Delta \bar{U}_{i}\right|=O\left(\left|x-x^{i}\right|\right)$ for all $i$. This proves that if $x-x^{i}$ is small, $\mathcal{R} P_{u}\left(x, 0^{-}\right)+\mathcal{R} P_{s}\left(x, 0^{+}\right)=\operatorname{span}\left(U_{1}+\Delta U_{1} \ldots U_{n}+\Delta U_{n} \bar{U}_{2}+\Delta \bar{U}_{2} \ldots \bar{U}_{n}+\Delta \bar{U}_{n}\right)$, depends smoothly on $x$. The adjoint equation of (7.19) can be found in (7.6) where $V=V^{i}, q=q^{i}$. Let $\left(\psi_{i 1}(x), \psi_{i 2}(x)\right)$ be the unique bounded solution to the adjoint equation with $\left|\psi_{i 2}(0)\right|=1$. Let $U_{0}=\left(\psi_{i 1}(0), \psi_{i 2}(0)\right)$ that is orthogonal to $\mathcal{R} P_{u}\left(0^{-}\right)+\mathcal{R} P_{s}\left(0^{+}\right)$. By a standard projection method, there exists a unique $\Delta U_{0} \in \mathcal{R} P_{u}\left(x, 0^{-}\right)+\mathcal{R} P_{s}\left(x, 0^{+}\right)$such that $U_{0}+\Delta U_{0}$ is orthogonal to $\mathcal{R} P_{u}\left(x, 0^{-}\right)+$ $\mathcal{R} P_{s}\left(x, 0^{+}\right)$. It can be shown that (7.6) has a bounded solution with the initial data $U_{0}+\Delta U_{0}$, cf. [18]. A normalized solution $\left(\psi_{i 1}(0, x), \psi_{i 2}(0, x)\right)$ with the initial data $\left(\psi_{i 1}(0, x), \psi_{i 2}(0, x)\right),\left|\psi_{i 2}(0, x)\right|=1$, can obtained by rescaling. Let $\psi_{i}(0, x)=$ $\psi_{i 2}(0, x)$. This proves the smooth dependence of $\psi_{i}(0, x)$ on $x$.

(iv) From Lemma 2.9, (b), $\sigma\left\{L_{x}^{i}\right\} \cap\left\{\operatorname{Re} \lambda>-\sigma_{0}+\eta_{0}\right\}$ consists of isolated eigenvalues of finite order. When $x=x^{i}, V^{i}(x)=0$, from $\mathrm{H} 5, \lambda=0$ is a simple 
eigenvalue, all the other eigenvalues satisfy $\operatorname{Re} \lambda<-\alpha_{0}$. Since eigenvalues depend continuously on $x$. Thus, if $0<\bar{\alpha}_{0}=\min \left\{\alpha_{0}, \sigma_{0}-\eta_{0}\right\}$, there exists $\epsilon>0$ such that if $\left|x-x^{i}\right|<\epsilon$ then $\lambda=0$ is the only eigenvalue in $\left\{\operatorname{Re} \lambda>-\bar{\alpha}_{0}\right\}$ and is simple.

(v) From H6, (4.7) is valid if $x=x^{i}$. Since both integrals in (4.7) depend continuously on $x$, thus (4.7) is valid for $\left|x-x^{i}\right|<\epsilon$ if $\epsilon>0$ is small. The formula for $\frac{\partial V^{i}\left(x^{i}\right)}{\partial x}$ follows from (7.18).

Proof of Lemma 4.2. $L_{x}^{i}$ is a Fredholm operator in $E_{\mathbb{R}}^{m}(w)$ with index zero. From Lemma 4.1, (iii), $\operatorname{Ker}\left\{L_{x}^{i}\right\}=\operatorname{span}\left\{q_{\xi}^{i}(\cdot, x)\right\}$ is one-dimensional. Therefore

$$
\text { Range }\left\{L_{x}^{i}\right\}=\left\{\psi_{i}(\cdot, x)\right\}
$$

is of codimension one. Consider the mapping $\mathcal{F}:\left(x, u, V_{1}\right) \rightarrow(g, h)$ as follows

$$
\begin{aligned}
& L_{x}^{i} u-V_{1} q_{\xi}^{i}(\cdot, x)=g, \\
& \left\langle u, q_{\xi}^{i}(\cdot, x)\right\rangle=h .
\end{aligned}
$$

$\mathcal{F}: O^{i} \times E_{\mathbb{R}}^{m+2}(w) \times \mathbb{R} \rightarrow E_{\mathbb{R}}^{m}(w) \times \mathbb{R}$ is $C^{\infty}$, in fact, linear with respect to $u \in E_{\mathbb{R}}^{m+2}(w)$ and $V_{1} \in \mathbb{R}$. It can be verified that $\partial \mathcal{F} / \partial\left(u, V_{1}\right)$ is a linear homeomorphism in the indicated norms. We only need to show that $\left(u, V_{1}\right)$ is uniquely solvable from (7.20) for any $(g, h) \in E_{\mathbb{R}}^{m}(w) \times \mathbb{R}$. If we choose $V_{1}=\left\langle\psi_{i}(\cdot, x), g\right\rangle$. $\left\langle\psi_{i}(\cdot, x), q_{\xi}^{i}(\cdot, x)\right\rangle^{-1}$, then $g-V_{1} q_{\xi}^{i} \in$ Range $\left\{L_{x}^{i}\right\}$. Any two solutions of the first of (7.20) differ by a multiple of $q_{\xi}^{i} \in \operatorname{Ker}\left\{L_{x}^{i}\right\}$, that can be determined by the second of (7.20). Let $h=0$ and denote the solutions by $V_{1}=V_{*}^{i}(x, g)$ and $u=u(\cdot, x, g)$.

The smoothness of $V_{*}^{i}(x, g)$ and $u(\cdot, x, g)$ on $(x, g)$ also follows from the Implicit Function Theorem applied on the function $\mathcal{F}$.

Proof of Lemma 5.1. According to Lemma 2.9, (b), $\mathbb{C}_{1}=\sigma\left\{L_{q^{i}}\right\} \cap\left\{\operatorname{Re} \lambda>-\partial_{0}+\right.$ $\left.\eta_{0}\right\}$ consists of only eigenvalues. When $i=0, r$, from $\mathrm{H} 5, L_{q^{i}}$ has no eigenvalues in $\mathbb{C}_{1}$ in the space $C_{b u}\left(\mathbb{R}^{+}, \mathbb{R}^{n}\right)$. Thus, it also has no eigenvalues in $\mathbb{C}_{1}$ in the space $B_{\mathbb{R}^{ \pm}}(w)$. This proves the case $i=0, r$. When $1 \leq i \leq r-1$, from H5 again, in $C_{b u}\left(\mathbb{R}, \mathbb{R}^{n}\right)$, the only eigenvalue of $L_{q^{i}}$ in $\mathbb{C}_{1}$ is $\lambda=0$, simple. Thus the only eigenvalue of $L_{q^{i}}$ in $B_{\mathbb{R}}(w)$ is also $\lambda=0$, simple. From Lemma 2.8, $q^{i}\left(\cdot, \bar{\eta}^{i}\right)$ is asymptotically stable modulo spatial shifts.

Proof of Lemma 5.2. The proof is exactly like that of Lemma 5.1.

\section{REFERENCES}

1. N. Alikakos, P. Bates and G. Fusco, Slow motion for the Cahn-Hilliard equation in one space dimension, J. Differential Equations 90 (1991), 81-135. MR 92a:35152

2. S. B. Angenent, J. Mallet-Paret, and L. A. Peletier, Stable transition layers in a semilinear boundary value problem, J. Differential Equations, 67 (1987), 212-242. MR 88d:34018

3. J. Carr and R. L. Pego, Metastable patterns in solutions of $u_{t}=\epsilon^{2} u_{x x}-f(u)$, Comm. Pure. Appl. Math. 42 (1989), 523-576. MR 90f:35091

4. S.-N. Chow, J. Hale and J. Mallet-Paret, An example of bifurcation to homoclinic orbits, J. Differential Equations, 37 (1980), 351-373. MR 81m:58056

5. G. Da Prato and P. Grisvard, Equations d'evolution abstraites non linéaires de type parabolique, Ann. Mat. Pura. Appl., 120 (1979), 329-396. MR 81d:34052

6. W. Eckhaus, Matching principles and composite expansions, Lecture Notes in Mathematics 594, Springer-Verlag, New York, 1977, 146-177. MR 58:6857

7. W. Eckhaus, Asymptotic analysis of singular perturbations, North-Holland, Amsterdam, 1979. MR 81a:34048

8. John W. Evans, Nerve Axon Equations: I Linear Approximations, Indiana Univ. Math. J., 21 (1972), 877-885. MR 45:1616 
9. John W. Evans, Nerve Axon Equations: II Stability at Rest, Indiana Univ. Math. J., 22 (1972), 75-90. MR 48:1729

10. John W. Evans, Nerve Axon Equations: III Stability of the Nerve Impulse, Indiana Univ. Math. J., 22 (1972), 577-593. MR 52:14697

11. John W. Evans, Nerve Axon Equations: IV The Stable and the Unstable Impulse, Indiana Univ. Math. J., 24 (1975), 1169-1190. MR 52:14698

12. P. C. Fife, Pattern formation in reacting and diffusing systems, J. Chem. Phys., 64 (1976), 554-564.

13. P. C. Fife, Singular perturbation and wave front techniques in reaction-diffusion problems, SIAM-AMS Proceedings, 10 (1976),23-50. MR 58:25358

14. P. C. Fife, Dynamics of internal layers and diffusive interfaces, CBMS-NSF, Regional Conference Series in Applied Mathematics, 53, SIAM, 1988. MR 90c: 80012

15. P. C. Fife, Diffusive waves in inhomogeneous media, Proc. Edinburgh Math. Soc., 32 (1989), 291-315. MR 90j:35109

16. P. C. Fife and L. Hsiao, The generation of solutions of internal layers, J. Nonlinear Anal. TMA 12 (1988), 19-41. MR 89c:35078

17. G. Fusco and J. Hale, Slow motion manifolds, dormant instability, and singular perturbations, J. Dynamics and Differential Equations 1 (1989), 75-94. MR 90i:35131

18. J. K. Hale and X.-B. Lin, Heteroclinic orbits for retarded functional differential equations, J. Differential Equations 65 (1986), 175-202. MR 88g:34113

19. J. K. Hale and K. Sakamoto, Existence and stability of transition layers, Japan Journal of Appl. Math. 5 (1988), 367-405. MR 90a:35112

20. C. Jones, Stability of the travelling wave solution of the FizHugh-Nagumo system, Trans. Amer. Math. Soc. 286 (1984), 431-469. MR 86b:35011

21. C. Jones and N. Kopell, Tracking invariant manifolds with differential forms in singularly perturbed systems, J. Differential Equations, 108 (1994), 64-88.

22. X.-B. Lin, Shadowing lemma and singularly perturbed boundary value problems, SIAM J. Appl. Math., 49 (1989), 26-54. MR 90a:34126

23. X.-B. Lin, Heteroclinic bifurcation and singularly perturbed boundary value problems, J. Differential Equations, 84 (1990), 319-382. MR 91d:34055

24. A. Lunardi, On the evolution operator for abstract parabolic equations, Israel J. Math., 60 (1987), 281-314. MR 89f:47066

25. Y. Nishiura and H. Fujii, Stability of singularly perturbed solutions to systems of reactiondiffusion equations, SIAM J. Math. Anal. 18 (1987), 1726-1770. MR 88j:35089

26. K. J. Palmer, Exponential dichotomies and transversal homoclinic points, J. Differential Equations 55 (1984), 225-256. MR 86d:58088

27. A. Pazy, Semigroups of linear operators and applications to partial differential equations, Springer, New York, 1983. MR 86b:47075

28. D. Sattinger, On the stability of travelling waves, Adv. Math., 22 (1976), 312-335. MR 55:8561

29. D. Sattinger, Weighted norms for the stability of travelling waves, J. Differential Equations, 25 (1977), 130-144. MR 56:6123

30. M. Schechter, Principle of functional analysis, Academic Press, New York and London, 1971. MR 56:3607

31. E. Sinestrari, On the abstract Cauchy problem of parabolic type in spaces of continuous functions, J. Math. Anal. Appl., 107 (1985), 16-66. MR 86g:34086

Department of Mathematics, North Carolina State University, Raleigh, North CarOLINA 27695-8205

E-mail address: xblin@xblsun.math.ncsu.edu 